-

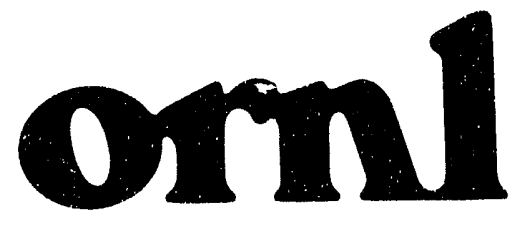

OAK RIDGE NATIONAL LABORATORY

MAGTH MARIETRA

\title{
A Detailed Study of Nonperturbative Solutions of Two-Body Dirac Equations
}

\author{
H. W. Crater \\ R. L. Becker \\ C. Y. Wong \\ P. Van Alstine
}


This report has been reproduced directly from the best available copy.

Available to DOE and DOE contractors from the Office of Scientific and Technical Information, P.O. Box 52, Oak Ridge, TN 37831; prices available from (615) $576-8401$, FTS $626-8401$.

Available to the public from the National Technical Information Service, U.S. Department of Commerce, 5285 Port Royal Rd., Springfield, VA 22161.

This report was prepared as an account of work sponsored by an agency of the United States Government. Neither the United States Government nor any agency thereof, nor any of their employees, makes any warranty, express or implied, or assumes any legal liability or responsibility for the accuracy, com. pleteness, or usefulness of any information, apparatus, product, or process disclosed, or represents that its use would not infringe privately owned rights. Reference herein to any specific commercial product, process, or service by trade name, trademark, manufacturer, or otherwise, does not necessarily constitute or imply its endorsement, recommendation, or favoring by the United States Government or any agency thereot. The views and opinions of authors expressed herein do not necessarily state or reflect those of the United States Government or any agency thereof. 
Contract No. DE-AC05-840R21400

PHYSICS DIVISION

\section{A DETAILED STUDY OF NONPERTURBATIVE SOLUTIONS OF TWO-BODY DIRAC EQUATIONS}

H. W. Crater, R. L. Becker, C. Y. Wong, and P. Van Alstine

Date Published - December 1992

OAK RIDGE NATIONAL LABORATORY

Oak Ridge, Tennessee 37831

managed by
MARTIN MARIETTA ENERGY SYSTEMS, INC.

for the

DEPARTMENT OF ENERGY 


\begin{abstract}
In quark model calculations of the meson spectrum, fully covariant two-body Dirac equations dictated by Dirac's relativistic constraint mechanics gave a good fit to the entire meson mass spectrum for light quark mesons as well as heavy quark mesons (excluding flavor mixing) with constituent world scalar and vector potentials depending on just one or two parameters. In this paper, we investigate the properties of these equations that made them work so well by solving them numerically for quantum electrodynamics (QED) and related field theories. The constraint formalism generates a relativistic quantum mechanics defined by two coupled Dirac equations on a sixteen component wave function which contain Lorentz covariant constituent potentials that are initially undetermined. An exact Pauli reduction leads to a second order relativistic Schrödinger-like equation for a reduced eight component wave function determined by an effective interaction - the quasipotential. We first determine perturbatively to lowest order the relativistic quasipotential for the Schrödinger-like equation by comparing that form with one derived from the Bethe-Salpeter equation. Insertion of this perturbative information into the minimal interaction structures of the two-body Dirac equations then completely determines their interaction structures. Then we give a procedure for constructing the full sixteen component solution to our coupled first-order Dirac equations from a solution of the second order equation for the reduced wave function. Next, we show that a perturbative treatment of these equations yields the standard spectral results for QED and related interactions. The relativistic potentials in our
\end{abstract}


exact Schrödinger-like equations incorporate detailed minimal interaction and dynamical recoil effects characteristic of field theory yet, unlike the approximate Fermi-Breit forms, do not lead to singular wave functions for any angular momentum states. Hence, we are able to solve them numerically and compare the resultant nonperturbative energy eigenvalues to their perturbative counterparts and hence to standard field theoretic results. We find that, unlike what occurs in the solution of the Breit equation, nonperturbative solution of our equation produces energy levels that agree with the perturbative spectrum through order $\alpha^{4}$. Surprisingly, this agreement depends crucially on inclusion of coupling between upper-upper and lower-lower components of our 16-component Dirac wave functions and on the short distance behavior of the relativistic quasipotential in the associated Schrödinger-like equation. To examine speculations that the effective potentials (including the angular momentum barrier) for some states in the $e^{+} e^{-}$system may become attractive for small separations, we study whether our equations predict pure QED resonances in the $e^{+} e^{-}$system which might correspond to the anomalous positron peaks in the yield of $e^{+} e^{-}$pairs seen in heavy-ion collisions. For the ${ }^{3} P_{0}$ state we find that, even though the quasipotential becomes attractive at separations near $10 \mathrm{fm}$ and overwhelms the centrifugal barrier, the attraction is not strong enough to hold a resonance. This result contradicts recent predictions of such states by other authors based on numerical solutions of three dimensional truncations of the Bethe-Salpeter equation for which the QED bound state wave equation has only been treated successfully by perturbation theory. 


\section{Introduction}

Recent quark model calculations of the meson spectrum ${ }^{1-2}$ using fully covariant two-body Dirac equations ${ }^{3-5}$ derived by Crater and Van Alstine from Dirac's relativistic constraint dynamics ${ }^{6-10}$ gave a good description of the light quark as well as the heavy quark meson masses resulting from world scalar and vector potentials. Although static potentials that have a close connection with quantum chromodynamics (QCD) such as the Adler-Piran potential ${ }^{11}$, or the cruder Richardson potential ${ }^{12}$, were responsible for the quality of the fit to the heavy mesons, the good quality of the simultaneous fit to the lighter mesons ( with the same one or two potential parameters used for the entire spectrum ) was due to exact two-body relativistic kinematics combined with the minimal interaction structure of these equations for vector and scalar potentials. In particular the structure of the vector potentials in these equations was originally abstracted from the classical electrodynamics of Wheeler and Feynman by two of us $^{13}$. We shall show in this paper that this structure may be obtained from QED by first deriving the Todorov quasipotential equation $^{13-14}$ from the Bethe-Salpeter equation and then comparing it to the two-body Dirac equations. One may formulate these quantum mechanical equations for semi-phenomenological meson studies with interactions taken from QCD or for electrodynamic bound state calculations with interactions dictated by QED. Since the abelian vector structure $\mathrm{e}^{15}$ of electrodynamics carries over to the short distance structure of QCD, in order that the equations be appropriate for QCD bound state calculations, they must give correct answers to the appropriate order in the fine structure constant $\alpha$ when applied to QED bound states. In previous 
work, Crater and Van Alstine have been able to solve analytically the full sixteen component coupled Dirac equations for the electrodynamic case to obtain a family of exact solutions for parapositronium ${ }^{\mathbf{1 6}}$, with energy spectrum in agreement with standard approaches to QED through order $\alpha^{4}$. Does the agreement with QED extend to unequal masses and to all angular momentum states? If so, this agreement would constitute the first successful test of the strong potential structure of two-body relativistic wave equations for QED for states of arbitrary angular momentum. It is imperative that such a test be done in order to discover whether a nonperturbative treatment of these or any other candidate equations faithfully represents the field theoretic dynamics obtained rigorously from perturbation theory as in QED or semi-phenomenologically from QCD. In order to carry out this check, we first treat our "minimal interaction constraint equations" perturbatively for the electromagnetic interaction. We show that they yield the correct two-body spectrum through order $\alpha^{4}$ when one treats as perturbations to the static Coulomb potential the various corrections of order $\frac{1}{c^{2}}$ generated by the spin structure of the Dirac equations alone. Unlike the equations produced by other approaches, the Schrödinger-like form of our two-body Dirac equations possess local spin-dependent and Darwin terms that are quantum mechanically well defined. Since our equations are devoid of highlysingular effective potential terms that appear in most three dimensional truncations of the Bethe-Salpeter equation and in the Breit equation ${ }^{17}$, we can go on to solve our equations nonperturbatively.

We shall demonstrate in this paper that a numerical solution of the two-body Dirac equations of constraint dynamics yields energies for 
the $n=1,2,3$ levels of fermion-anti-fermion systems in QED that agree through order $\alpha^{4}$ with those produced by a perturbative treatment of these equations and with those produced by standard perturbative approaches to QED. Furthermore, as a check on the scalar and time-like vector interactions for our equations, we shall demonstrate nonperturbatively that our equations yield no hyperfine splitting for those interactions, in agreement with a perturbative treatment. In each case we shall treat the general unequal mass system, including only the potentials that arise from the single exchange diagram and ignoring the contribution of the virtual annihilation diagram to the equal mass case.

Crater and Van Alstine originally abstracted the electrodynamic vector interaction in these equations from classical electrodynamics in order to describe the semi-phenomenological short range interactions of $\mathrm{QCD}^{1-2}$. Because numerical solution of our equations reproduces the standard perturbative bound state spectrum of QED, we have a set of two-body relativistic wave equations for electrodynamics whose nonperturbative predictions for other phenomena in QED ought to be taken as seriously if not more seriously than those of other field theoretic equations that have not been similarly checked. Just such a situation presents itself in the interpretation of recent results in heavy ion physics. Wong and Becker ${ }^{18}$ have speculated that the unexplained anomalous peaks in the yield of $e^{+} e^{-}$pairs in heavy ion collisions ${ }^{19-24}$ might result from purely QED resonances ${ }^{25-26}$ in the $e^{+} e^{-}$system produced by strong potential electrodynamic structures in the appropriate two-body wave equation. If there is such a resonance, first one must study it using a wave equation ${ }^{27}$ and second this wave equation must be treated nonper- 
turbatively and covariantly, not by perturbative, semirelativistic means. In this paper we investigate what the two-body Dirac equations have to say about such states. The fact that in our equations (in Schrödinger-like form) each term of the quasipotential is quantum mechanically well defined all the way into the origin is critical to our investigation. We make a numerical search for resonances in the ${ }^{3} P_{0}$ continuum states of positronium. We show that numerical calculation of the phase shift for energies of $1.4-1.8 \mathrm{Mev}$ agree with perturbatively computed phase shifts. Thus, we find theoretical evidence that no such resonances exist in our electrodynamic constraint equations. We find that even though the local QED quasipotential for the ${ }^{3} P_{0}$ state becomes attractive at small distances and overwhelms the centrifugal barrier as in the model of Wong and Becker $^{18}$, the QED quasipotential is not deep or wide enough to hold a resonance. This result directly contradicts the results obtained by Vary and Spence ${ }^{28}$ from standard non-local truncations of the Bethe-Salpeter equation.

As we shall see in this paper, when we solve the two-body Dirac equations numerically, we find that relativistic potential structures that do not contribute in the usual perturbation theory play a significant role. What is the origin of these structures in two-body Dirac equations? The basic relativistic interaction in our equations is determined by the BetheSalpeter equation via the Feynman scattering amplitudes of the relevant quantum field theory. The resulting two-body Dirac equations then assurne different forms depending in part on the Lorentz character of the chosen field-theoretic interaction and in part on the spin structure dictated by the mathematical compatibility of the two coupled wave equa- 
tions. Together these nonperturbative requirements completely specify spin dependence. Our equations inherit the basic potential structure of the single particle Dirac equation corrected by recoil terms dictated by compatibility (a relativistic version of Newton's third law ${ }^{5}$ ). The requirement of compatibility also automatically controls the relative time by forcing its elimination from the invariant potential in the center of momentum (c.m.) frame.

Later in this paper, we shall recast the two-body Dirac equations for electrodynamics into the Schrödinger-like form:

$$
\left(p^{2}+\Phi_{w}(\mathcal{A})-\left(\epsilon_{w}^{2}-m_{w}^{2}\right)\right) \psi=0
$$

in which $\Phi_{w}(\mathcal{A})$ is a sixteen by sixteen component c.m. energy $(w)$ dependent, relativistic quasipotential matrix, dependent on an invariant function $\mathcal{A}$ derived from field theory at the lowest order. Those terms in Eq.(1.1) in $\Phi_{w}$ beyond the collective minimal (Todorov) form ${ }^{29}$ $2 \epsilon_{w} \mathcal{A}-\mathcal{A}^{2}$ (see section II for a definition of $m_{w}$ and $\epsilon_{w}$ ) we will call "strong potential" terms. The role played by these terms can be fully investigated only by nonperturbative means (for example, through numerical solution of the resultant eigenvalue equation). In past work ${ }^{1-2}$ on two-body Dirac equations, we had tacitly assumed (along with authors of all other treatments of the Bethe-Salpeter equation of which we are aware) that a full nonperturbative, numerical treatment of the equations would yield standard spectral results since the "weak-potential" form of the equations (including the usual $\frac{1}{r^{3}}$ and delta function potentials) reduced to a form ${ }^{14,30}$ known to generate the standard spectral results. But, in view of the failure of another two-body equation - the Breit equation - to generate its own perturbative results when some of 
the Breit terms are included nonperturbatively ${ }^{31-32}$, can we trust this assumption? If it were not true for a particular equation when applied to the vector interaction of perturbative $Q E D$, how could we trust results produced by that particular equation in a purely nonperturbative application (dominated by a relatec vector interaction) such as to the quark-antiquark bound states of QCD. Any candidate two-body wave equation, applied to QCD with such an interaction, must reproduce, if applied to QED, the perturbative QED spectrum when that equation is treated nonperturbatively regardless of the agreement of its semiphenomenological spectrum with the meson spectrum.

The ordinary one-body Dirac equation with external Coulomb potential certainly yields agreement between nonperturbative solution and perturbative evaluation. In that case, the exact solution produces a spectrum that agrees through order $\alpha^{4}$ with that given by perturbative treatment of the Darwin and spin-orbit terms obtained from the usual Pauli reduction of the Dirac equation. As two of us found in a previous paper $^{16}$, the two-body Dirac equations of constraint dynamics for the $e^{+} e^{-}$system in the ${ }^{1} J_{J}$ states also possess a family of exact solutions with total c.m. energy $w$ given by a Sommerfeld formula -

$$
\begin{aligned}
w & =m \sqrt{2+2 \sqrt{1+\frac{\alpha^{2}}{\left(n+\sqrt{\left(l+\frac{1}{2}\right)^{2}-\alpha^{2}}-l-\frac{1}{2}\right)^{2}}}} \\
& =2 m-\frac{m \alpha^{2}}{4 n^{2}}-\frac{m \alpha^{4}}{2 n^{3}(2 l+1)}+\frac{11}{64} \frac{m \alpha^{4}}{n^{4}}+O\left(\alpha^{6}\right) .
\end{aligned}
$$

These energies are in agreement through order $\alpha^{4}$ with those of the perturbative solution of the same equation and also with those of standard 
approaches to QED. As we shall see the two-body Dirac equations of QED extend this agreement to the $n=1,2,3$ levels for all allowable $j$ and unequal masses. This agreement has not been demonstrated for the traditional three dimens. nnal truncations of the Bethe-Salpeter equation ${ }^{17}$. Such truncations do yield the correct QED spectrum for fermion-antifermion systems through order $\alpha^{4}$ (from single photon exchange) when treated perturbatively. In all of these traditional treatments, one starts with a bound state Coulomb wave function ( whether non-relativistic or relativistic) and uses frst order perturbation theory to compute Breit corrections corresponding to Darwin, spin-orbit, spin-spin, and tensor interactions. However, these three dimensional truncations have not been solved analytically or numerically for $\mathrm{QED}^{33}$ with enough accuracy to demonstrate agreement with a perturbative treatment of these equations through order $\alpha^{4}$.

Our paper is organized as follows. In section II we review the constraint formalism for the two-body Dirac equations containing mutual world scalar and vector potentials. Then in section III we show how we obtain the relativistic interactions of our equations from the appropriate perturbative quantum field theory in concert with the minimal interaction structures of the two-body Dirac equations in both their constituent Dirac and collective Schrödinger forms. This procedure determines the quasipotential $\Phi_{w}$ of Eq.(1.1).

In section IV, from the coupled Dirac equations, we derive an eight component Schrödinger-like form of the equations, that we later solve numerically. In the process we show how to use the solutions of the Schrödinger-like equations to construct the full sixteen component solu- 
tions of the two original Dirac equations. In section $\mathrm{V}$ we give a perturbative treatment of the weak potential form of these equations for later comparison with nonperturbative solution. In section VI we arrive at the first nonperturbative numerical result of this paper. There, we examine the eigenvalues obtained from numerical solution of the Schrödinger-like forms derived in section IV and compare these with the corresponding perturbative results of section $V$. In each case, we find that the nonperturbative bound state spectrum produced by solution of the fully coupled system of equations equals the perturbative results within an error of order $\alpha^{6}$. We find that the coupling between upper-upper and lower-lower parts of the sixteen component wave functions in our equations is crucial to this agreement. This dependence is unexpected since that coupling does not contribute through order $\alpha^{4}$ in the perturbative evaluation of these equations. Moreover, we find that the parts of the quasipotential essential for agreement with the perturbative results become significant only at separations on the order of a few fermis. Thus, insofar as the order $\alpha^{4}$ spectral results are concerned, these two-body Dirac equations give correct results when used well below the Compton wavelength. This agreement allows us to test with confidence the hypothesis of possible $e^{+} e^{-}$resonances in the ${ }^{3} P_{0}$ state. In section VII we use a further decoupling of the equations, derived in the appendix, to compute phase shifts using both perturbative and nonperturbative treatments. We find no evidence for a pure QED resonance in the $e^{+} e^{-}$ system, in direct contradiction to the results of Spence and Vary ${ }^{28}$. Finally in section VIII we compare various properties of our two-body Dirac approach with those of other relativistic two-body wave equations. 


\section{Review of Two-Body Dirac Equations for Two Spin-} One-Half Particles For World Scalar and Four-Vector Interactions

A. "External Potential" or "Minimal Interaction" Forms of the Two-Body Dirac Equations

We begin by examining explicit covariant forms of the two-body Dirac equations ${ }^{3-5}$ that two of us have developed for us $\ldots$ semiphenomenological meson-spectroscopy calculations ${ }^{1-2}$ and for investigations of the electromagnetic positronium system ${ }^{16}$. For two relativistic spinone-half particles interacting through scalar and vector potentials, the two compatible, 16-component (or $4 \times 4$ matrix) Dirac equations ${ }^{1-5}$ of constraint dynamics are

$$
\begin{aligned}
& \mathcal{S}_{1} \psi \equiv \gamma_{51}\left(\gamma_{1} \cdot\left(p_{1}-A_{1}\right)+m_{1}+S_{1}\right) \psi=0 \\
& \mathcal{S}_{2} \psi \equiv \gamma_{52}\left(\gamma_{2} \cdot\left(p_{2}-A_{2}\right)+m_{2}+S_{2}\right) \psi=0 .
\end{aligned}
$$

The subscript $i=1,2$ stands for the $i$ th particle so that $m_{1}$, and $m_{2}$ are the masses of the interacting fermions. In Eqs.(2.1a-b) the potentials $A_{i}^{\mu}$ and $S_{i}$ introduce the interactions that the $i t h$ particle experiences due to the presence of the other particle. (Thus we will refer to these forms of the two-body Dirac equations either as the "external potential forms" or the "minimal interaction forms".) In meson calculations motivated by QCD the Lorentz invariant scalar potentials $S_{i}$ are semiphenomenological while the vector potentials $A_{i}^{\mu}$ are composed of two independent covariant parts, one semi-phenomenological (long range and confining) like the scalar interactions, and the other (short range) closely 
tied to perturbative quantum field theory. The first part contains only long range time-like pieces (parallel to the total four-momentum of the two particles), while the second is electromagnetic-like (short range), containing field theoretically specified portions of time-like and spacelike pieces (transverse to the total four-momentum of the two particles). The specific forms of the covariant spin-dependent terms in the interactions are consequences of the necessary compatibility of the two Dirac equations

$$
\left[\mathcal{S}_{1}, \mathcal{S}_{2}\right] \psi=0 .
$$

In detail ${ }^{2,5,16}$ the vector potentials $A_{i}^{\mu}$ are given in terms of three invariant functions $G, E_{1}$ and $E_{2}$ by (with $\partial_{\mu} \equiv \partial / \partial x^{\mu}$ )

$$
\begin{aligned}
& A_{1}^{\mu}=\left(\left(\epsilon_{1}-E_{1}\right)-i \frac{G}{2} \gamma_{2} \cdot\left(\frac{\partial E_{1}}{E_{2}}+\partial \ln G\right) \gamma_{2} \cdot \hat{P}\right) \hat{P}^{\mu}+(1-G) p^{\mu}-\frac{i}{2} \partial G \cdot \gamma_{2} \gamma_{2}^{\mu} \\
& A_{2}^{\mu}=\left(\left(\epsilon_{2}-E_{2}\right)+i \frac{G}{2} \gamma_{1} \cdot\left(\frac{\partial E_{2}}{E_{1}}+\partial \ln G\right) \gamma_{1} \cdot \hat{P}\right) \hat{P}^{\mu}-(1-G) p^{\mu}+\frac{i}{2} \partial G \cdot \gamma_{1} \gamma_{1}^{\mu}
\end{aligned}
$$

while the scalar potentials $S_{i}$ are functions of $G$ and two additional invariant functions $M_{1}$ and $M_{2}$

$$
\begin{aligned}
S_{1} & =M_{1}-m_{1}-\frac{i}{2} G \gamma_{2} \cdot \frac{\partial M_{1}}{M_{2}} \\
S_{2} & =M_{2}-m_{2}+\frac{i}{2} G \gamma_{1} \cdot \frac{\partial M_{2}}{M_{1}} .
\end{aligned}
$$

In the case of lowest order QED, $S_{i}=0$, and the space-like and time-like vectors are not independent but combine into the electromagnetic-like four-vectors

$$
\begin{aligned}
& A_{1}^{\mu}=\left(\epsilon_{1}-\frac{G\left(\epsilon_{1}-\epsilon_{2}\right)}{2}+\frac{\left(\epsilon_{1}-\epsilon_{2}\right)}{2 G}\right) \hat{P}^{\mu}+(1-G) p^{\mu}-\frac{i}{2} \partial G \cdot \gamma_{2} \gamma_{2}^{\mu} \\
& A_{2}^{\mu}=\left(\epsilon_{2}-\frac{G\left(\epsilon_{2}-\epsilon_{1}\right)}{2}+\frac{\left(\epsilon_{1}-\epsilon_{2}\right)}{2 G}\right) \hat{P}^{\mu}-(1-G) p^{\mu}+\frac{i}{2} \partial G \cdot \gamma_{1} \gamma_{1}^{\mu},
\end{aligned}
$$


In Eqs.(2.3a-b) and Eqs.(2.5a-b) the variable

$$
P=p_{1}+p_{2}
$$

is the total four-momentum. In our metric $-P^{2}=w^{2}$ is the c.m. energy squared so that $\hat{P}^{2}=-1$ where $\hat{P} \equiv P / w$. The variables $\epsilon_{i}$ are the conserved c.m. energies of the constituent particles given by

$$
\epsilon_{1}=\left(w^{2}+m_{1}^{2}-m_{2}^{2}\right) / 2 w, \epsilon_{2}=\left(w^{2}+m_{2}^{2}-m_{1}^{2}\right) / 2 w
$$

so that $\epsilon_{1}+\epsilon_{2}=w$. In terms of these energies the usual relative momentum defined by $p_{1}=\epsilon_{1} \hat{P}+p, p_{2}=\epsilon_{2} \hat{P}-p$ becomes

$$
p=\left(\epsilon_{2} p_{1}-\epsilon_{1} p_{2}\right) / w
$$

In order that equations (2.1a) and (2.1b) be compatible (i.e. satisfy $(2.2))$ it is necessary that the invariant functions $E_{1}, E_{2}, G, M_{1}$ and $M_{2}$ depend on the relative separation, $x=x_{1}-x_{2}$, only through the spacelike coordinate four vector ${ }^{7-9}$

$$
x_{\perp}^{\mu}=x_{\mu}+\hat{P}^{\mu}(\hat{P} \cdot x)
$$

which is perpendicular to the total four-momentum, $P$. In general $E_{1}, E_{2}, G, M_{1}$, and $M_{2}$ may depend on

$$
x_{\perp}^{2} \equiv r^{2}, l^{2}=l_{\mu} l^{\mu}, \text { and } p_{\perp}^{2}
$$

where $l_{\mu}=\epsilon_{\mu \nu \kappa \lambda} \hat{P}^{\nu} x_{\perp}^{\kappa} p_{\perp}^{\lambda}$. Note that the invariant $r$ is the interparticle separation in the c.m. system. In this paper we shall assume that the invariant functions depend only on $r$. In general $E_{1}, E_{2}$ and $G$ are related 
to each other ${ }^{13,5}$ and for QCD applications are functions of only two invariant functions, $\mathcal{V}(r)$ and $\mathcal{A}(r)$, whose forms we take to be

$$
\begin{aligned}
& E_{1}^{2}(\mathcal{A}, \mathcal{V})=G^{2}\left(\left(\epsilon_{1}-\mathcal{A}\right)^{2}-2 \epsilon_{w} \mathcal{V}+\mathcal{V}^{2}\right) \\
& E_{2}^{2}(\mathcal{A}, \mathcal{V})=G^{2}\left(\left(\epsilon_{2}-\mathcal{A}\right)^{2}-2 \epsilon_{w} \mathcal{V}+\mathcal{V}^{2}\right)
\end{aligned}
$$

and

$$
G^{2}=\frac{1}{(1-2 \mathcal{A} / w)}
$$

From the expressions $(2.3 \mathrm{a}-\mathrm{b}$ and $2.5 \mathrm{a}-\mathrm{b})$ of the vector potentials we see that the invariant function $\mathcal{A}(r)$ is responsible for the covariant electromagnetic-like parts of $A_{i}^{\mu}$ while $\mathcal{V}$ is responsible for the additional independent covariant time-like parts of $A_{i}^{\mu}$. Even though the dependences of $E_{1}, E_{2}$, and $G$ on $\mathcal{A}$ and $\mathcal{V}$ are not unique, they are constrained by the requirement that they yield the correct non-relativistic and semirelativistic limits. Demanding that the Schrödinger form of the two-body Dirac equations incorporate the collective minimal (Todorov) interaction structures ${ }^{29}$ of Eq.(1.1), we find the simple forms given in Eqs.(2.11a-c) satisfy these requirements. (The details of this argument are given in Refs. $(5,13,34))$. In general $M_{1}$ and $M_{2}$ are related to each other $^{4-5}$ and for QCD applications are functions of the two invariant functions $\mathcal{A}(r)$ and $S(r)$ :

$$
\begin{aligned}
& M_{1}^{2}(\mathcal{A}, S)=m_{1}^{2}+G^{2}\left(2 m_{w} S+S^{2}\right) \\
& M_{2}^{2}(\mathcal{A}, S)=m_{2}^{2}+G^{2}\left(2 m_{w} S+S^{2}\right) .
\end{aligned}
$$

The invariant function $S(r)$ is primarily responsible for the scalar potentials since $S_{i}=0$ if $S(r)=0$ while $\mathcal{A}(r)$ contributes to the $S_{i}$ (if $S(r) \neq 0$ ) 
as well as to the vector potentials $A_{i}^{\mu}$. Demanding that the Schrödinger form of the two-body Dirac equations incorporate the collective minimal (Todorov) interaction structures we find that the simple forms given in F qs.(2.12a-b) give the correct non-relativistic and semirelativistic limits. (The details of this argument are likewise given in Refs.(4-5,34)). Thus the five invariant functions $M_{1}, M_{2}, E_{1}, E_{2}$, and $G$ are constrained to depend on three independent invariant functions $S, \mathcal{A}$, and $\mathcal{V}$. (In QED applications, $\mathcal{V}=0$ and in lowest order $S=0$ ). The kinematical variables

$$
\begin{gathered}
m_{w}=m_{1} m_{2} / w \\
\epsilon_{w}=\left(w^{2}-m_{1}^{2}-m_{2}^{2}\right) / 2 w
\end{gathered}
$$

are the relativistic reduced mass and energy of a fictitious particle of relative motion. The corresponding value of the on-mass-shell relative momentum squared then takes the form

$$
b^{2}(w) \equiv\left(w^{4}-2 w^{2}\left(m_{1}^{2}+m_{2}^{2}\right)+\left(m_{1}^{2}-m_{2}^{2}\right)^{2}\right) / 4 w^{2}=\epsilon_{w}^{2}-m_{w}^{2} .
$$

For the electromagnetic-like vector interactions the minimal interaction form of the two-body Dirac equations (2.1a-b) is a consequence of gauge invariance. In any one-body wave equation, gauge invariance exhibits itself in two related ways. For the system of particle and field, (Abelian) gauge invariance manifests itself as invariance under change of the vector field by the addition of the gradient of an arbitrary scalar combined with local phase variation of the wave function. This is achieved through the derivative structure of the field equations in concert with the minimal coupling of the potential to particle. However, once one eliminates the vector potentials in terms of source motions in a fixed gauge, 
the minimal structures persist as dynamical structures of the resulting particle equations. For example, in the case of a single charged particle interacting with an infinitely heavy massive charge, the resulting KleinGordon or Dirac equation, with Coulomb potential obtained from the full particle plus field problem by elimination of the field potential in a fixed gauge, contains the dynamical potential as a minimal subtraction from the energy and retains the phase change with a compensating addition of the gradient of a scalar to the vector potential minimally subtracted from the momentum. Thus, "gauge invariance" of the resulting particle equations is a dynamical symmetry inherited from the original system of particle and field through the elimination of the vector field in a fixed gauge.

Similarly, the two-body Dirac equations (2.1a-b) contain vector potentials (one for each particle) obtained from quantum field theory from the Bethe-Salpeter equation in the Feynman gauge ${ }^{35}$ (see section III below) or from classical field theory in the Lorentz gauge. Thus, the two-body Dirac equations are two-body counterparts of the one-body particle equations with eliminated field and should possess an analogous inherited dynamical "gauge invariance" if they retain any invariance at all. In fact, we find that since our equations are two simultaneous wave equations on one wave function with two (albeit related) four-potentials, Eqs.(2.1a-b) turn out to be invariant under any gauge transformation of the form $A_{i}^{\mu} \rightarrow A_{i}^{\mu}+\partial_{i}^{\mu} \chi\left(x_{\perp}\right)$ with $\chi$ the phase change of the single wave function. The origin of the two dynamical potentials $A_{1}^{\mu}$ and $A_{2}^{\mu}$ as solutions for vector fields in the Lorentz gauge shows up as the property

$$
\frac{\partial}{\partial x^{\mu}}\left(A_{1}^{\mu}+A_{2}^{\mu}\right)=0
$$


which is a consequence of the fact that $A_{1}^{\mu}+A_{2}^{\mu} \propto \hat{P}^{\mu} f$ where $f=f\left(x_{\perp}\right)$. This property of the potentials is forced upon us by the compatibility (2.2) of the constraints.

B. Hyperbolic Forms of the Two-Body Dirac Equations

The expansions (2.10) and (2.11) for the five invariant functions in terms of the three invariants $\mathcal{A}\left(x_{\perp}\right), \mathcal{V}\left(x_{\perp}\right), S\left(x_{\perp}\right)$ are important for semiphenomenological and other applications that emphasize the relationship of the interactions in our equations to external potentials of the two associated one-body problems. However, for applications in which the identification of these five invariants in terms of either a perturbative or semi-phenomenological field theoretic scattering amplitude is desirable, two of us have found a hyperbolic representation ${ }^{36}$ of these five invariants in terms of three other invariants, $L, J$, and $\mathcal{G}$. This representation is $(c h \equiv \cosh , s h \equiv \sinh )$

$$
\begin{gathered}
M_{1}=m_{1} \operatorname{ch} L+m_{2} \operatorname{sh} L \\
M_{2}=m_{2} \operatorname{ch} L+m_{1} \operatorname{sh} L \\
E_{1}=\epsilon_{1} \operatorname{chJ}+\epsilon_{2} \operatorname{sh} J \\
E_{2}=\epsilon_{2} \operatorname{chJ}+\epsilon_{1} \operatorname{shJ} \\
G=e^{\mathcal{G}} .
\end{gathered}
$$

$L\left(x_{\perp}\right), J\left(x_{\perp}\right)$, and $\mathcal{G}\left(x_{\perp}\right)$ generate scalar, time-like vector and space-like vector interactions respectively. As shown in the next section, this representation puts the two-body equations in a form whose interactions are 
simply related to the Bethe-Salpeter equation via the Feynman scattering amplitude. If we use (2.16) and the "theta" matrices

$$
\begin{gathered}
\theta_{i}^{\mu} \equiv i \sqrt{\frac{1}{2}} \gamma_{5 i} \gamma_{i}^{\mu}, \mu=0,1,2,3, i=1,2 \\
\theta_{5 i} \equiv i \sqrt{\frac{1}{2}} \gamma_{5 i}
\end{gathered}
$$

we can rewrite $(2.1 \mathrm{a}-\mathrm{b})$ as

$$
\begin{gathered}
\mathcal{S}_{1} \psi=\left(G \theta_{1} \cdot p+E_{1} \theta_{1} \cdot \hat{P}+M_{1} \theta_{51}\right. \\
\left.+i G\left(\theta_{2} \cdot \partial \mathcal{G} \theta_{1 \perp} \cdot \theta_{2 \perp}+\theta_{2} \cdot \partial J \theta_{1} \cdot \hat{P} \theta_{2} \cdot \hat{P}-\theta_{2} \cdot \partial L \theta_{51} \theta_{52}\right)\right) \psi=0 \\
\mathcal{S}_{2} \psi=\left(-G \theta_{2} \cdot p+E_{2} \theta_{2} \cdot \hat{P}+M_{2} \theta_{52}\right. \\
\left.-i G\left(\theta_{1} \cdot \partial \mathcal{G} \theta_{1 \perp} \cdot \theta_{2 \perp}+\theta_{1} \cdot \partial J \theta_{1} \cdot \hat{P} \theta_{2} \hat{P}-\theta_{1} \cdot \partial L \theta_{51} \theta_{52}\right)\right) \psi=0
\end{gathered}
$$

Simplification of these equations results if we introduce the following invariant matrix functions $\Delta_{k}\left(x_{\perp}\right)$, with $k=L, J, \mathcal{G}$. For scalar interactions

$$
\Delta_{L}=-\frac{\mathcal{O}_{1} L\left(x_{\perp}\right)}{2}=-\frac{I_{1} I_{2} L\left(x_{\perp}\right)}{2} \mathcal{O}_{1}
$$

where $I_{1}$ and $I_{2}$ are the identity operators and $\mathcal{O}_{1}=2 \theta_{51} \theta_{52}$. For timelike vector interactions

$$
\Delta_{J}=\frac{\mathcal{O}_{2} J\left(x_{\perp}\right)}{2}=\frac{\gamma_{1} \cdot \hat{P} \gamma_{2} \cdot \hat{P} J\left(x_{\perp}\right)}{2} \mathcal{O}_{1}
$$

where $\mathcal{O}_{2}=2 \theta_{1} \cdot \hat{P} \theta_{2} \cdot \hat{P}$, and for space-like vector interactions

$$
\Delta_{\mathcal{G}}=\frac{\mathcal{O}_{3} \mathcal{G}\left(x_{\perp}\right)}{2}=\frac{\gamma_{1 \perp} \cdot \gamma_{2 \perp} \mathcal{G}\left(x_{\perp}\right)}{2} \mathcal{O}_{1}
$$


where $\mathcal{O}_{3}=2 \theta_{1 \perp} \cdot \theta_{2 \perp}$. For convenience we define

$$
\begin{gathered}
\Delta=\Delta_{J}+\Delta_{L}+\Delta_{\mathcal{G}} \\
=\frac{1}{2}\left(\mathcal{O}_{2} J\left(x_{\perp}\right)-\mathcal{O}_{1} L\left(x_{\perp}\right)+\mathcal{O}_{3} \mathcal{G}\left(x_{\perp}\right)\right)
\end{gathered}
$$

In terms of these matrix functions, the compatible two-body Dirac equations become

$$
\begin{gathered}
\mathcal{S}_{1} \psi=\left(\exp (\mathcal{G}) \theta_{1} \cdot p+\operatorname{ch}\left(2 \Delta_{J}\right) \epsilon_{1} \theta_{1} \cdot \hat{P}+\operatorname{sh}\left(2 \Delta_{J}\right) \epsilon_{2} \theta_{2} \cdot \hat{P}\right. \\
\left.+m_{1} \operatorname{ch}\left(2 \Delta_{L}\right) \theta_{51}+m_{2} \operatorname{sh}\left(2 \Delta_{L}\right) \theta_{52}+\exp (\mathcal{G}) i \theta_{2} \cdot \partial \Delta\right) \psi=0 \\
\mathcal{S}_{2} \psi=\left(-\exp (\mathcal{G}) \theta_{2} \cdot p+\operatorname{ch}\left(2 \Delta_{J}\right) \epsilon_{2} \theta_{2} \cdot \hat{P}+\operatorname{sh}\left(2 \Delta_{J}\right) \epsilon_{1} \theta_{1} \cdot \hat{P}\right. \\
\left.+m_{2} \operatorname{ch}\left(2 \Delta_{L}\right) \theta_{52}+m_{1} \operatorname{sh}\left(2 \Delta_{L}\right) \theta_{51}-\exp (\mathcal{G}) i \theta_{1} \cdot \partial \Delta\right) \psi=0 .
\end{gathered}
$$

Remarkably, the linear combinations

$$
\begin{aligned}
& \mathbf{S}_{1} \psi=\left(\operatorname{ch}(\Delta) \mathcal{S}_{1}-\operatorname{sh}(\Delta) \mathcal{S}_{2}\right) \psi=0 \\
& \mathbf{S}_{2} \psi=\left(\operatorname{ch}(\Delta) \mathcal{S}_{2}-\operatorname{sh}(\Delta) \mathcal{S}_{1}\right) \psi=0
\end{aligned}
$$

of the constraint equations given in (2.23a-b) have very simple forms. Since $\mathcal{O}_{1}^{2}=\mathcal{O}_{2}^{2}=\frac{1}{4}\left(\mathcal{O}_{1} \mathcal{O}_{2}-\mathcal{O}_{3}\right)^{2}=1$ we are able to use various hyperbolic identities to simplify $(2.24 \mathrm{a}-\mathrm{b})$. In particular, by bringing the matrices on the left of each $\mathcal{S}_{i}$ to the right we find that ${ }^{36}$

$$
\begin{aligned}
& \mathbf{S}_{1} \psi=\left(\mathcal{S}_{10} \operatorname{ch}(\Delta)+\mathcal{S}_{20} \operatorname{sh}(\Delta)\right) \psi=0 \\
& \mathbf{S}_{2} \psi=\left(\mathcal{S}_{20} \operatorname{ch}(\Delta)+\mathcal{S}_{10} \operatorname{sh}(\Delta)\right) \psi=0 .
\end{aligned}
$$

(One can even start from free Dirac equations in the form of Eqs.(2.25ab) with constant $\Delta$ and introduce interactions by "gauging", i.e. letting 
$\Delta$ become point dependent ${ }^{36}$.) In Eqs. $(2.25 \mathrm{a}-\mathrm{b}) \mathcal{S}_{10}$ and $\mathcal{S}_{20}$ are the free Dirac operators ${ }^{37}$

$$
\begin{aligned}
& \mathcal{S}_{10}=\theta_{1} \cdot p_{1}+m_{1} \theta_{51}=\theta_{1} \cdot p+\epsilon_{1} \theta_{1} \cdot \hat{P}+m_{1} \theta_{51} \\
& \mathcal{S}_{20}=\theta_{2} \cdot p_{2}+m_{2} \theta_{52}=-\theta_{2} \cdot p+\epsilon_{2} \theta_{2} \cdot \hat{P}+m_{2} \theta_{52}
\end{aligned}
$$

In $(2.1 \mathrm{a}-\mathrm{b})$ (or $(2.18 \mathrm{a}-\mathrm{b}))$ the relativistic potentials are two-body analogs of, and in the limit $m_{1} \rightarrow \infty$ (or $m_{2} \rightarrow \infty$ ) go over to, the ordinary external potentials of the one-body Dirac equation. The Lorentz character of these interactions is apparent from the "external potential" or minimal interaction form of the equations. On the other hand, the hyperbolic forms $(2.25 \mathrm{a}-\mathrm{b})$ display the Lorentz character of the interaction through the gamma matrix structures of the scalar $\Delta$. These matrix structures of its Lorentz invariant terms are dictated either by the perturbative agreement of the hyperbolic interactions with the corresponding interactions of the Bethe-Salpeter equation or by phenomenological considerations. Eqs.(2.25a-b) are closely related to another form of the two-body Dirac equations introduced by Sazdjian ${ }^{38}$. In the notation used here his equations are

$$
\begin{aligned}
& \left(\mathcal{S}_{10}+\mathcal{S}_{20} \Delta\right) \psi=0 \\
& \left(\mathcal{S}_{20}+\mathcal{S}_{10} \Delta\right) \psi=0
\end{aligned}
$$

The Sazdjian equations are equivalent to ours in the reak-potential limit ${ }^{39}$.

We use the forms $(2.25 \mathrm{a}-\mathrm{b})$ to relate the matrix potentials $\Delta$ to a given field theoretic or semi-phenomenological 16 by 16 matrix Feynman amplitude. For example, a matrix amplitude proportional to $\gamma_{1}^{\mu} \gamma_{2 \mu}$ 
corresponding to an electromagnetic-like interaction would according to (2.20) and (2.21) dictate that $J=-\mathcal{G}$ (see section III below). Matrix amplitudes proportional to either $I_{1} I_{2}$ or $\gamma_{1} \cdot \hat{P} \gamma_{2} \cdot \hat{P}$ would correspond to semi-phenomenological scalar or time-like vector interactions. The hyperbolic forms (2.25a-b) of the two-body Dirac equations lead to a particularly simple version ${ }^{36}$ for the norm of the sixteen component Dirac spinor. On the other hand the minimal interaction or "external potential" forms (2.1a-b) (or (2.18a-b)) of the two-body Dirac equations are simpler to reduce to the Schrödinger-like forms most useful for numerical calculations of bound and scattering states. 


\section{Field Theoretic Identification of the Quasipotential}

In the quark model calculations for meson spectroscopy described in Refs.(1-2) the identifications of the invariant forms $\mathcal{V}, S$, and $\mathcal{A}$ or $L, J$, and $\mathcal{G}$ were taken from static potentials obtained from an educated guess, (Richardson's potential ${ }^{12}$ ) or from an effective nonlinear classical field theory based on mean field approximations to QCD (the AdlerPiran potential $\left.{ }^{11}\right)$. In contrast, for QED we obtain the invariant form of the quasipotential $\Phi_{w}$ directly from field theory. In this section we show how the invariant function $\mathcal{A}$ contained within $\Phi_{w}$ is obtained from lowest order QED. Before doing this for the Dirac equations we review the constraint equations for spinless bosons to guide our effort

In recent work ${ }^{2}$ two of us used Sazdjian's "quantum mechanical transform" 40 of the Bethe-Salpeter wave function to derive the "quasipotential equation" of Todorov ${ }^{14}$ from a field theory for spinless particles. The Todorov quasipotential equation is an inhomogeneous integral equation which relates the quasipotential $\Phi_{w}$ appearing in a Schrödinger-like, three-dimensional equation

$$
\left(p_{\perp}^{2}+\Phi_{w}\left(x_{\perp}, p_{\perp}\right)\right) \psi_{w}\left(x_{\perp}\right)=b^{2}(w) \psi_{w}\left(x_{\perp}\right)
$$

to certain matrix elements of the off-mass-shell, field theoretic, relativistic scattering amplitude. It is closely connected to the present work through Eq.(3.1) which it shares with constraint dynamics.

A. The Quasipotential Equation for Spinless Particles

The two, coupled, Klein-Gordon equations of constraint dynamics ${ }^{7-10,5,13}$ 
can be written as

$$
\mathcal{H}_{i} \psi_{w} \equiv\left(p_{i}^{2}+m_{i}^{2}+\Phi_{i}\right) \psi_{w}=0, i=1,2
$$

with

$$
P^{2} \psi_{w}=-w^{2} \psi_{w}
$$

The compatibility requirement $\left[\mathcal{H}_{1}, \mathcal{H}_{2}\right] \psi_{w}=0$ implies $^{7-11,4-5,13}$ that if $\Phi_{1}=\Phi_{2} \equiv \Phi_{w}$ then

$$
\frac{1}{2}\left(\mathcal{H}_{1}-\mathcal{H}_{2}\right) \psi_{w}=\frac{1}{2}\left(\left(p_{1}^{2}-p_{2}^{2}\right)-\left(m_{2}^{2}-m_{1}^{2}\right)\right) \psi_{w} \equiv P \cdot p \psi_{w}=0
$$

Thus, even though $\psi_{w}$ is off mass-shell it does satisfy $\left(p_{1}^{2}+m_{1}^{2}\right) \psi_{w}=\left(p_{2}^{2}+\right.$ $\left.m_{2}^{2}\right) \psi_{w} \cdot^{8,13}$. The right hand side of (3.4) implies that in the c.m. system the wave function is independent of the relative time $\left(x_{\perp}=(0, \vec{r})\right)$. A second independent combination of the constraints $\mathcal{H}_{1}$ and $\mathcal{H}_{2}$,

$$
w^{-1}\left(\epsilon_{2} \mathcal{H}_{1}+\epsilon_{1} \mathcal{H}_{2}\right) \psi_{w}=\left(p_{\perp}^{2}+\Phi_{w}\left(x_{\perp}, p_{\perp}\right)-b^{2}(w)\right) \psi_{w}\left(x_{\perp}\right)=0
$$

determines this off-shell behavior through the quasipotential $\Phi_{w}$. For scattering states in an arbitrary Lorentz frame

$$
\psi_{P, q_{\perp}}^{(+)}(X, x)=\frac{e^{i P \cdot X}}{(2 \pi)^{4}} \psi_{w, q_{\perp}}^{(+)}\left(x_{\perp}\right)
$$

with

$$
\begin{gathered}
\psi_{w, q_{\perp}}^{(+)}\left(x_{\perp}\right)=\psi_{w, q_{\perp}}^{(0)}\left(x_{\perp}\right)-\left(2 w\left(p_{\perp}^{2}-b^{2}(w)-i \epsilon\right)\right)^{-1} V_{w}\left(x_{\perp}, p_{\perp}\right) \psi_{w, q_{\perp}}^{(+)}\left(x_{\perp}\right) \\
w \geq m_{1}+m_{2}
\end{gathered}
$$

where

$$
\psi_{w, q_{\perp}}^{(0)}\left(x_{\perp}\right) \equiv(2 \pi)^{-3} e^{i q_{\perp} \cdot x_{\perp}}
$$


with $q_{\perp}^{2}=b^{2}(w)$ and $V_{w} \equiv 2 w \Phi_{w}$. The corresponding momentum space wave function is given by the four dimensional Fourier transform

$$
\int d^{4} x \psi_{w, q_{\perp}}^{(+)}\left(x_{\perp}\right) e^{-i k \cdot x}=2 \pi \delta(\hat{P} \cdot k) \tilde{\psi}_{w, q_{\perp}}^{(+)}\left(k_{\perp}\right)
$$

where $\tilde{\psi}_{w, q_{\perp}}^{(+)}\left(k_{\perp}\right)$ is itself given in terms of the position space wave function by the covariant three dimensional transform

$$
\tilde{\psi}_{w, q_{\perp}}^{(+)}\left(k_{\perp}\right)=\int d^{4} x \delta(\hat{P} \cdot x) \psi_{w, q_{\perp}}^{(+)}\left(x_{\perp}\right) e^{-i k \cdot x}
$$

whose inverse transform is

$$
\psi_{w, q_{\perp}}^{(+)}\left(x_{\perp}\right)=\int \frac{d^{4} k}{(2 \pi)^{3}} c(\hat{P} \cdot k) \tilde{\psi}_{w, q_{\perp}}^{(+)}\left(k_{\perp}\right) e^{i k \cdot x} .
$$

Eq.(3.7) then yields

$$
\tilde{\psi}_{w, q_{\perp}}^{(+)}\left(k_{\perp}\right)=\delta^{3}\left(k_{\perp}-q_{\perp}\right)-\frac{\int d^{3} k_{\perp}^{\prime} \tilde{\mathbf{V}}_{w}\left(k_{\perp}, k_{\perp}^{\prime}\right) \tilde{\psi}_{w, q_{\perp}}^{(+)}\left(k_{\perp}^{\prime}\right)}{\left(2 w(2 \pi)^{3}\left(k_{\perp}^{2}-b^{2}(w)-i \epsilon\right)\right)}
$$

in which

$$
\delta^{3}\left(p_{\perp}-q_{\perp}\right) \equiv \int d(\hat{P} \cdot p) \delta^{4}(p-q)
$$

and

$$
\int d^{3} k_{\perp}^{\prime} \equiv \int d^{4} k^{\prime} \delta\left(\hat{P} \cdot k^{\prime}\right)
$$

with $\tilde{\mathbf{V}}$ related to the Fourier transform of $V_{w}\left(x_{\perp}, p_{\perp}\right)$ by

$$
\tilde{\mathbf{V}}_{w}\left(k_{\perp}, k_{\perp}^{\prime}\right)=\tilde{V}_{w}\left(k_{\perp}-k_{\perp}^{\prime}, k_{\perp}^{\prime}\right)
$$

Note that the momentum space constraint wave function is not $\psi_{w, q_{\perp}}^{(+)}\left(k_{\perp}\right)$ but rather $\delta(\hat{P} \cdot k) \tilde{\psi}_{w, q_{\perp}}^{(+)}\left(k_{\perp}\right)$. 
If we define the scattering amplitude $\mathbf{T}_{\boldsymbol{w}}\left(p_{\perp}, \boldsymbol{q}_{\perp}\right)$ in the usual way

$$
\mathbf{T}_{w}\left(p_{\perp}, q_{\perp}\right) \equiv-\int d^{3} p_{\perp}^{\prime} \tilde{\mathbf{V}}_{w}\left(p_{\perp}, p_{\perp}^{\prime}\right) \tilde{\psi}_{w, q_{\perp}}^{(+)}\left(p_{\perp}^{\prime}\right)
$$

multiply Eq.(3.12) by $-\tilde{\mathrm{V}}_{w}$, and integrate we are led to a LippmannSchwinger equation for this amplitude in terms of the quasipotential $\tilde{\mathbf{V}}_{w}$ $\mathbf{T}_{w}\left(p_{\perp}, q_{\perp}\right)+\tilde{\mathbf{V}}_{w}\left(p_{\perp}, q_{\perp}\right)+\int \frac{d^{3} p_{\perp}^{\prime}}{(2 \pi)^{3}} \frac{\tilde{\mathbf{V}}_{w}\left(p_{\perp}, p_{\perp}^{\prime}\right)}{2 w\left(p_{\perp}^{2}{ }^{\prime}-b^{2}(w)-i \epsilon\right)} \mathbf{T}_{w}\left(p_{\perp}^{\prime}, q_{\perp}\right)=0$

Symbolically this equation is of the form

$$
\mathbf{T}_{w}+\mathrm{V}_{w}+\mathrm{V}_{w} G_{\perp} \mathbf{T}_{w}=0
$$

in which $G_{\perp}$ stands for

$$
G_{w}^{(+)}\left(p_{\perp}\right)=\frac{1}{(2 \pi)^{3} 2 w\left(p_{\perp}^{2}-b^{2}-i \epsilon\right)} .
$$

The scattering amplitude then automatically satisfies the elastic twobody unitarity condition ${ }^{41}$

$\mathbf{T}_{w}\left(p_{\perp}, q_{\perp}\right)-\mathbf{T}_{w}^{*}\left(q_{\perp}, p_{\perp}\right)=\frac{\pi i}{w} \int \frac{d^{3} k_{\perp}}{(2 \pi)^{3}} \mathbf{T}_{w}^{*}\left(k_{\perp}, p_{\perp}\right) \delta\left(k_{\perp}^{2}-b^{2}\right) \mathbf{T}_{w}\left(k_{\perp}, q_{\perp}\right)$.

The Lippmann-Schwinger equation (3.16) gives the relativistic quantum mechanical scattering amplitude $\mathbf{T}_{\boldsymbol{w}}$ in terms of a prescribed quasipotential $\tilde{\mathbf{V}}_{w}$. Eq.(3.16) is Todorov's inhomogeneous quasipotential equation. However, that equation is usually solved for $\tilde{\mathbf{V}}_{w}$ in terms of a $\mathbf{T}_{\boldsymbol{w}}$ which is identified with the field theoretic scattering amplitude $T_{w}\left(p_{\perp}, q_{\perp}\right)$. In Appendix $A$ we present an explicit momentum space derivation of the 
Todorov inhomogeneous quasipotential from the inhomogeneous Bethe-Salpeter equation $\left(p_{1}=\epsilon_{1} \hat{P}+k, p_{2}=\epsilon_{2} \hat{P}-k\right)$

$$
\begin{gathered}
T_{w}(p ; q)=K_{w}(p ; q) \\
-\frac{i}{(2 \pi)^{4}} \int d^{4} k K_{w}(p ; k) G_{1}^{(+)}\left(\epsilon_{1} \hat{P}+k\right) G_{2}^{(+)}\left(\epsilon_{2} \hat{P}-k\right) T_{w}(k ; q)
\end{gathered}
$$

(relating the Bethe-Salpeter kernel $K_{w}(p, q)$ that plays the role of the potential in the homogeneous Bethe Salpeter equation to $T_{w}\left(p_{\perp}, q_{\perp}\right)$ ). Like the formal operator derivation given earlier ${ }^{2}$ by two of us, it uses Sazdjian's quantum mechanical transform of the Bethe-Salpeter wave function. However, the new derivation shows the connection with earlier three dimensional approaches ${ }^{41-42}$ and emphasizes the role of elastic two-body unitarity.

To summarize, the two constraint equations (3.4) and (3.5) play two different roles. Eq.(3.4) forces the relative energy (in the c.m. system) to vanish while the Schrödinger-like equation (3.5) describes the effect of the dynamics and puts the system on a collective mass-shell (of total energy $w$ in the c.m.). Other than the requirement that the constraint potential $\Phi_{w}$ depend on $x$ only through $x_{\perp}$, the constraint equations give no further restriction on the dynamical content of the constraint potential (for spinless particles). When constraint dynamics is being used in conjunction with quantum field theory, the potential $\Phi_{w}$ can be determined from an appropriate quantum field theory by way of (3.16) (Eq.(A.19) in Appendix A or in terms of Eqs.(A.17-18)). When the field theoretic starting point is the Bethe-Salpeter equation, the connection must be made through an object - the Sazdjian projection - in which the relative time (about which nothing is said in the Bethe-Salpeter equation) is eliminated as in (3.4) or (A.12). Thus, one starts from the 
Bethe-Salpeter equation (3.19) and ends with the constraint equation (3.1) with the constraint potential $\Phi_{w}$ determined from (3.16), or (A.18) and (A.19).

Transformations from the two-time four dimensional Bethe-Salpeter equation to one-time three dimensional quasipotential equations have a long history dating back to early work of Logunov and Tavkhelidze $\mathrm{e}^{42}$ and Blankenbecler and Sugar ${ }^{43}$. In subsequent papers Yaes ${ }^{17}$ and Gross ${ }^{17}$ pointed out that there are in fact an infinite number of such three dimensional reductions of the Bethe-Salpeter equation. The equations presented here and that of Sazdjian are particular cases, motivated by constraint dynamics, that lead to simple Schrödinger-like wave equations.

\section{B. The Quasipotential Equation for Two Spin - $\frac{1}{2}$ - Particles}

\section{Constraint Dynamics}

When spin is included we describe the quantum system in terms of two compatible Dirac equations (2.25a-b). At this stage we are only interested in first order field theoretic amplitudes. For these, our equations are approximately the weak potential forms

$$
\begin{aligned}
& \mathbf{S}_{1} \psi=\left(\mathcal{S}_{10}+\mathcal{S}_{20} \Delta^{(1)}\right) \psi=0 \\
& \mathbf{S}_{2} \psi=\left(\mathcal{S}_{20}+\mathcal{S}_{10} \Delta^{(1)}\right) \psi=0
\end{aligned}
$$

which are Sazdjian's forms of the two-body Dirac equations ${ }^{38}$.

Now we obtain from these two equations forms analogous to those we used in the spinless case. First, because $\left[\mathcal{S}_{10}, \mathcal{S}_{20}\right]=0$, we find that

$$
\left(\mathcal{S}_{10} \mathbf{S}_{1}-\mathcal{S}_{20} \mathbf{S}_{2}\right) \psi=-P \cdot p \psi=0
$$


Now since $P \cdot p \psi=0$, we have

$$
-2 \mathcal{S}_{10}^{2} \psi=-2 \mathcal{S}_{20}^{2} \psi=\left(p_{\perp}^{2}-b^{2}\right) \psi
$$

As a result, we find that

$$
-2 \mathcal{S}_{10} \mathbf{S}_{1} \psi=-2 \mathcal{S}_{20} \mathbf{S}_{2} \psi=\left(p_{\perp}^{2}-b^{2}+\Phi_{w}^{(1)}\right) \psi=0
$$

where

$$
\Phi_{w}^{(1)}=-2 \mathcal{S}_{10} \mathcal{S}_{20} \Delta^{(1)}
$$

which relates Sazdjian's $\Delta^{(1)}$ to the quasipotential of our relativistic Schrödinger equation.

2. Field Theory

In order to determine $\Phi_{w}^{(1)}$ and from it the corresponding $\Delta^{(1)}$, from field theory, we consider the inhomogeneous Bethe-Salpeter equation for two spin-one-half particles

$$
\begin{aligned}
T=K & +K\left(\left(\gamma_{1} p_{1}+m_{1}\right)\left(\gamma_{2} p_{2}+m_{2}\right)\right)^{-1} T \\
& =K+K \mathcal{S}_{10}^{-1} \mathcal{S}_{20}^{-1} \theta_{51} \theta_{52} T
\end{aligned}
$$

We remove the $\gamma$-matrices from the denominator. We let

$$
\mathcal{T}=4 \mathcal{S}_{10} \mathcal{S}_{20} \theta_{51} \theta_{52} T
$$

and

$$
\mathcal{K}=4 \mathcal{S}_{10} \mathcal{S}_{20} \theta_{51} \theta_{52} K
$$

Thus we have

$$
\mathcal{T}=\mathcal{K}+\mathcal{K} \frac{1}{4} \mathcal{S}_{10}^{-2} \mathcal{S}_{20}^{-2} \theta_{51} \theta_{52} T
$$




$$
=\mathcal{K}+\mathcal{K} G_{1}^{+} G_{2}^{+} \mathcal{T}
$$

where $G_{i}^{+} \equiv\left(p_{i}^{2}+m_{i}^{2}-i \epsilon\right)^{-1}$ is the Feynman propagator for the spinless case. Because of the similarity between the spinless equations (3.1) and (3.19) and the second order form of the spin-one-half equations (3.23) and (3.28), we can use the derivation of the Todorov inhomogeneous quasipotential equation for the spinless case given in Appendix $A$ to prove that the (lowest order) Sazdjian transform of the Bethe-Salpeter wave function for the spin-one-half, spin-one-half case is

$$
\left(p_{\perp}^{2}+\Phi_{w}^{(1)}\right) \Psi=b^{2}(w) \Psi
$$

but with an $x_{\perp}$ and spin dependent quasipotential

$$
\Phi_{w}^{(1)}=-\frac{\mathcal{T}^{(1)}}{2 w}
$$

Comparison of this with (3.24) identifies $\Delta^{(1)}$ as

$$
\Delta^{(1)}=2 \theta_{51} \theta_{52} \mathcal{T}^{(1)}
$$

We have shown how, in lowest order, the four dimensional BetheSalpeter equation can be transformed into the three dimensional Eq.(3.29). This equation is identical to the one obtained from the weak-potential constraint equations (3.20a-b). However, if we regard the field-theoretic connection not as a rigid one to the weak-potential Sazdjian form (3.20ab) but instead to the strong-potential constraint form (2.25a-b), (related to the "external potential" or "minimal interaction" constraint form by way of (2.24a-b)) those equations (Eqs.(2.25a-b)) clothe the (perturbative) field theoretic interactions in their own peculiar quantummechanical structures. These "strong-potential" structures appear in 
the dynamics of our wave equations (2.25a-b) through two-body potentials that treat each particle as though it were minimally coupled to an external potential (or potentials) generated by the other particle and in the hyperbolic structure of our equation through the occurrence of simple forms for the corresponding quantum mechanical norm ${ }^{36}$ of the wave function. These strong-potential structures $(2.25 \mathrm{a}-\mathrm{b})$ induce two difierent sorts of terms beyond those that appear in Sazdjian's (3.20ab). First, the nonlinear $\Delta$ terms in $\operatorname{ch} \Delta$ and $\operatorname{sh} \Delta$ produce additional spin dependences. Second, the quantity $\Delta$, through its dependence on the invariant potentials $L, J$, and $\mathcal{G}$, differs from $\Delta^{(1)}$ calculated in first order perturbation theory using (3.31). The invariants that appear in $\Delta^{(1)}$ are perturbative approximations of those that appear in $\Delta$. When one attempts to extrapolate the perturbative invariants above the order of approximation justified through comparison with the perturbative Bethe-Salpeter equation, those extrapolations are merely provisional subject to change when higher order field theoretic corrections in $\Delta$ are included (see (A.23) in Appendix A). However, nonperturbative principles like gauge invariance and our related demands of both constituent and collective forms of minimal interaction will constrain the forms of $\Delta$ that can appear in (2.25a-b). We shall show this for QED below.

C. The Case of Quantum Electrodynamics

For the electromagnetic interaction, the $T$ matrix in momentum space in the Feynman gauge is

$$
T_{w}^{(1)}(p, q)=\frac{e_{1} e_{2}}{(p-q)^{2}-i 0} \gamma_{1}^{\mu} \gamma_{2 \mu}
$$

where $p$ and $q$ are relative momenta. The simple form of the result- 
ing constraint equations eliminates the practical necessity in other approaches (for example the formalism of Caswell and Lepage ${ }^{35}$ ) of working in the Coulomb gauge. Because of the constraint (3.21), the coordinate space form of (3.29) becomes

$$
\left(p_{\perp}^{2} \cdots b^{2}-2 \mathcal{S}_{10} \mathcal{S}_{20} \theta_{51} \theta_{52} \frac{\gamma_{1}^{\mu} \gamma_{2 \mu} e_{1} e_{2}}{4 \pi w\left|x_{\perp}\right|}\right) \Psi=0
$$

Direct comparison of this form of the Bethe-Salpeter equation with the Schrödinger-like constraint form given in (3.23-24) yields

$$
\Delta^{(1)}=\theta_{51} \theta_{52} \gamma_{1}^{\mu} \gamma_{2 \mu} \frac{e_{1} e_{2}}{4 \pi w\left|x_{\perp}\right|}=\frac{e_{1} e_{2}}{4 \pi w\left|x_{\perp}\right|} \theta_{1} \cdot \theta_{2}
$$

Comparison of (3.34) with the definition of $\Delta$ in (2.22) shows: 1) $L^{(1)}\left(x_{\perp}\right)=$ 0 as expected, since a vector field theory cannot generate scalar potentials in lowest order, 2) $J^{(1)}=-\mathcal{G}^{(1)}$, which just tells us how the spaceand time-like vector portions are related for electromagnetic interactions, and finally, 3)

$$
\mathcal{G}^{(1)}\left(x_{\perp}\right)=\frac{e_{1} e_{2}}{4 \pi w\left|x_{\perp}\right|}
$$

Eqs.(2.11c) and (2.16e) of Sec. II imply that $\mathcal{A}^{(1)}=w \mathcal{G}^{(1)}$ so that

$$
\mathcal{A}^{(1)}\left(x_{\perp}\right)=\frac{e_{1} e_{2}}{4 \pi\left|x_{\perp}\right|}
$$

The nonperturbative extension of $\mathcal{G}$, or equivalently of the invariant function

$$
\mathcal{A}=\frac{w}{2}(1-\exp (-2 \mathcal{G}))
$$

(see (2.11c) and (2.16e)) is not determined by this comparison at orders beyond Eq.(3.35) or (3.36). However, $\mathcal{G}$ is restricted through gauge 
invariance as realized through the introduction of interactions through minimal substitution as done in the two-body Dirac equations Eqs.(2.1ab) of Sec. II. When restricted to electromagnetic-like interactions $(\mathcal{V}=$ $S=0$ ), these equations become the electromagnetic two-body Dirac equations

$$
\begin{aligned}
& \mathcal{S}_{1} \psi=\left(\pi_{1} \cdot \theta_{1}+m_{1} \theta_{51}\right) \psi=0 \\
& \mathcal{S}_{2} \psi=\left(\pi_{2} \cdot \theta_{2}+m_{2} \theta_{52}\right) \psi=0
\end{aligned}
$$

previously derived by two of $\mathrm{us}^{5,16}$ and solved analytically for the equal mass singlet case of positronium. In these equations the constituent vector potentials appear through the minimal substitutions

$$
\begin{gathered}
p_{1}^{\mu} \rightarrow \pi_{1}^{\mu} \equiv p_{1}^{\mu}-A_{1}^{\mu}=G\left(p^{\mu}+\left(\epsilon_{1}-\mathcal{A}\right) \hat{P}^{\mu}+i \theta_{2} \cdot \partial \mathcal{G} \theta_{2}^{\mu}\right) \\
p_{1}^{\mu} \rightarrow \pi_{1}^{\mu} \equiv p_{2}^{\mu}-A_{2}^{\mu}=G\left(-p^{\mu}+\left(\epsilon_{2}-\mathcal{A}\right) \hat{P}^{\mu}-i \theta_{1} \cdot \partial \mathcal{G} \theta_{1}^{\mu}\right)
\end{gathered}
$$

(see Eqs.(2.3a-b) and Eqs.(2.10a-c)) so that the squared forms of the constraints take the simple one-body electromagnetic-like form

$$
\begin{aligned}
& \mathcal{H}_{1} \psi \equiv-\frac{1}{2}\left[\mathcal{S}_{1}, \mathcal{S}_{1}\right]_{+} \psi=\left(\pi_{1}^{2}-\frac{1}{2} \sigma_{1}^{\mu \nu} F_{1 \mu \nu}+m_{1}^{2}\right) \psi=0 \\
& \mathcal{H}_{2} \psi \equiv-\frac{1}{2}\left[\mathcal{S}_{2}, \mathcal{S}_{2}\right]_{+} \psi=\left(\pi_{2}^{2}-\frac{1}{2} \sigma_{2}^{\mu \nu} F_{2 \mu \nu}+m_{2}^{2}\right) \psi=0
\end{aligned}
$$

where $F_{a \mu \nu} \equiv \frac{1}{i}\left[\pi_{\mu a}, \pi_{\nu a}\right]$. The difference of these two equations is $\left(\mathcal{H}_{1}-\right.$ $\left.\mathcal{H}_{2}\right) \psi=2 P \cdot p \psi=0$, just as in the spinless case. If we identify

$$
\mathcal{U}^{\mu} \equiv \frac{1}{i} \partial^{\mu} \mathcal{G}
$$

and write out only the spinless part of $\pi_{i}$, we find (see details in Sect. IV) that the weighted $\operatorname{sum} \mathcal{K} \psi=\left(\frac{\epsilon_{2}}{w} \mathcal{H}_{1}+\frac{\epsilon_{1}}{w} \mathcal{H}_{2}\right) \psi=0$ yields $\left(-\left(\epsilon_{w}-\mathcal{A}\right)^{2}+(p-\mathcal{U})^{2}+m_{w}^{2}+\right.$ spin-dependent + Darwin corrections $) \psi=0$. 
The spin-independent terms at the first part of the equation display Todorov's interpretation of system potentials as minimal extensions of the four momentum of relative motion $\left(\epsilon_{w}, \vec{p}\right)$. In fact, if we define $e^{44}$

$$
\mathcal{P}^{\mu}=\epsilon_{w} \hat{P}+p^{\mu}, A^{\mu}=\mathcal{A} \hat{P}^{\mu}+\mathcal{U}^{\mu},
$$

then the first part can be compactly written in the collective minimal (Todorov) interaction form $\left(\mathcal{P}^{\mu}-A^{\mu}\right)^{2}$ for the effective particle of relative motion.

What are the additional restrictions on $\mathcal{G}$ or $\mathcal{A}$ that arise from this collective minimal interaction form? The first restriction follows from the fact that the portion $2 \epsilon_{w} \mathcal{A}-\mathcal{A}^{2}$ of the quasipotential must be quantum mechanically well defined for a Schrödinger-like equation. This restricts $\mathcal{A}$ so that $-\mathcal{A}^{2}$ must not be singular $\left(<-\frac{1}{4 r^{2}}\right)$ as $r \rightarrow 0$. For example, the simple choice $\mathcal{G}=\mathcal{G}^{(1)}=e_{1} e_{2} / w r$ (corresponding to $\mathcal{A}=(w / 2)(1-$ $\left.\exp \left(-2 e_{1} e_{2} / w r\right)\right)$ would produce a $-\mathcal{A}^{2}$ term that grows exponentially as $r \rightarrow 0$ for $e_{1} e_{2}<0$ yielding an unacceptable singular behavior in the effective Schrödinger equation. The second restriction is that

$$
2 \epsilon_{w} \mathcal{A}-\mathcal{A}^{2}=2 \epsilon_{w} \mathcal{A}^{(1)}-\left(\mathcal{A}^{(1)}\right)^{2}+O\left(\mathcal{A}^{(1)}\right)^{3} .
$$

must be satisfied when $\mathcal{A}$ is expanded in powers of $\mathcal{A}^{(1)}$. Classically this restriction implies that when one carries out an expansion through order $1 / c^{2}$ by solving the minimal Todorov equation $p^{2}-\left(\epsilon_{w}-\mathcal{A}^{(1)}\right)^{2}+m_{w}^{2}=0$ for $w$, then one obtains an expansion that includes not only the standard relativistic corrections to the non-relativistic kinetic energy but also relativistic corrections ${ }^{13,34}$ to the non-relativistic potential that are canonically equivalent to the Darwin interaction. Thus this collective 
minimal interaction structure incorporates in a covariant way the Darwin interaction corrections to the non-relativistic potential $-\alpha / r$ without a complicated momentum dependence. These simple structures occur in Todorov's closely related quasipotential equation ${ }^{14}$ and in quantum constraint dynamics for spinless particles under vector interactions ${ }^{13}$. Using a scale transformation developed by Schwinger ${ }^{45}$, two of us have shown that ${ }^{5,13}$ this collective minimal structure yields an $O\left(1 / c^{2}\right)$ expansion that is canonically equivalent to the standard $O\left(1 / c^{2}\right)$ momentum dependent Darwin interaction

One solution to these two perturbative conditions on $\mathcal{A}$ is the naive identification $\mathcal{A}=\mathcal{A}^{(1)}$. This particular $\mathcal{A}$ provides a considerable simplification of the calculation of the semirelativistic (order $\alpha^{4}$ ) corrections to the QED bound state spectrum ${ }^{13-14}$ for spinless particles over the standard Breit related approaches. For this $\mathcal{A}$, Todorov et $\mathrm{al}^{30}$ have also shown how the $-\mathcal{A}^{2}$ terms correspond to higher order ladder, crossladder, and iterated exchange contributions to $\Phi_{w}$ (corresponding to segments of $\mathcal{G}^{(2)}$ in the notation of this paper). These constituent and collective minimal interaction requirements are nonperturbative ones beyond the strictly perturbative field theoretic restriction of (3.35) on $\mathcal{G}$. With the naive choice,

$$
\mathcal{A}=\mathcal{A}^{(1)}
$$

the nonperturbative extension of $\mathcal{G}$ implied by Eq.(3.37) is

$$
\mathcal{G}=-\frac{1}{2} \ln \left(1-\frac{2 e_{2} e_{2}}{w r}\right) \text {. }
$$

(Note again that the naive choice $\mathcal{G}=\mathcal{G}^{(1)}$ gives the correct lowest order $\mathcal{G}$ but would lead through Eq.(3.37) to an $\mathcal{A}$ that would produce a singular quasipotential.) 
In summary we have found three restrictions that $\mathcal{A}$ must satisfy. First, it must generate the correct lowest order interaction $\mathcal{G}^{(1)}$. Second it must generate the minimal Todorov form Eq. (3.44) in order to give the correct $O\left(1 / c^{2}\right)$ dynamics. Third, it must satisfy the nonperturbative restriction that $\mathcal{A}^{2}$ must not be too singular at $r=0$. As we anticipate, (see Sec. V) the above restrictions will guarantee that these nonperturbative potential forms will yield the correct results if the equations are treated perturbatively. A more crucial test of these nonperturbative or strong potential structures will be to determine if a nonperturbative (numerical) treatment of these equations will yield the correct spectrum to the appropriate order (see section $V I$ ).

D. Phenomenological Scalar and Vector Interactions.

To carry out semi-phenomenological applications of the constraint equations such as to the quark models of mesons, one does not perturbatively determine $\Delta$ from field theory as we did in (3.32-34). Two of us in Ref. 2 divided up the nonrelativistic static quark potential $U(r)$ in terms of the three invariants $\mathcal{A}(r), \mathcal{V}(r)$, and $S(r)$ of Eqs.(2.10-2.11) chosen so that

$$
\mathcal{A}(r)+\frac{1}{2}(\mathcal{V}(r)+S(r))=U(r) .
$$

This division of $U(r)$ was arbitrary, guided primarily by phenomenological considerations. However, this choice has consequences far beyond its nonrelativistic roots since the matrix structure to which it is attached dictates different relativistic Darwin and spin-dependent corrections depending on the corresponding matrix Lorentz invariant structures that 
appear in $\Delta$. In Ref. 2 we used

$$
\begin{gathered}
\Delta=\Delta_{J}+\Delta_{L}+\Delta_{\mathcal{G}} \\
=\frac{1}{2}\left(\mathcal{O}_{2} J\left(x_{\perp}\right)-\mathcal{O}_{1} L\left(x_{\perp}\right)+\mathcal{O}_{3} \mathcal{G}\left(x_{\perp}\right)\right) .
\end{gathered}
$$

in which from (2.16)

$$
\begin{gathered}
L=L(\mathcal{A}, S)=\ln \frac{\left(M_{1}+M_{2}\right)}{m_{1}+m_{2}} \\
J=J(\mathcal{A}, \mathcal{V})=\ln \frac{\left(E_{1}+E_{2}\right)}{\epsilon_{1}+\epsilon_{2}} \\
\mathcal{G}=\mathcal{G}(\mathcal{A})=\ln (G) .
\end{gathered}
$$

(We remind the reader that the "minimal interaction" form (2.18a-b) (depending on $\mathcal{A}, \mathcal{V}$, and $S$ ) of the two-body Dirac equations is equivalent to the hyperbolic form (2.25a-b) (depending on $L, J$, and $\mathcal{G}$ ), related by $(2.24 \mathrm{a}-\mathrm{b})$.) Note that for models with $\mathcal{V}=0$, our vector interaction is that used for the abelian interactions of lowest order QED. In the quark model applications, we used directly the "external potential" form (2.18a-b) with $\mathcal{A}, \mathcal{V}$, and $S$ identified as in Eq.(3.47). In contrast, for QED the form of $\mathcal{A}$ was dictated by the match between the $\dot{\Delta}$ of the weak potential form Eq. $(3.20 \mathrm{a}-\mathrm{b})$ on the one hand and the field theoretically derived quasipotential equation on the other. 


\section{Reduction of Two-Body Dirac Equations to Second- Order Relativistic Schrödinger-like Equations}

We wish to determine the total energy eigenvalues for the stationary states of two interacting spin - one half particles using the "external potential" or "minimal interaction" form of the two coupled Dirac equations Eqs.(2.18a-b). For this purpose, we have at our disposal the analogs of all of the decoupling procedures and simplifications resulting from special choices of Dirac matrix representations that one uses to solve the one-body Dirac equation. For example two of us $^{16}$ used the fact that $\sigma_{\mu \nu}$ is diagonal in the chiral representation to obtain exact solutions for bound electromagnetic equal-mass singlet states. In meson work $^{2}$, two of us decomposed the second-order equations corresponding to Eqs.(2.18a-b) (with gamma matrices in the Dirac representation) into four decoupled four-component second-order equations. Subsequently, Sazdjian pointed out ${ }^{45}$ to us that our reduction in the Dirac representation could only be carried out for singlet and $j=l$ triplet states, not for $j=l \pm 1$ triplet states (we review this development in appendix D of $\operatorname{Ref}(46)$.$) . Since the validity of that reduction turned out to be state-$ dependent, we replace it in this paper by a generally valid reduction of the coupled 16-component first order Eqs.(2.18a,b) to two decoupled eight-component second order Schrödinger-like equations

Since we shall work in a general frame, for convenir-nce we define the covariant versions of the standard Dirac $\vec{\alpha}$ and $\beta$ and $\vec{\Sigma}$ matrices for the two particles:

$$
\beta_{i} \equiv-\gamma_{i} \cdot \hat{P}=2 \theta_{5 i} \theta_{i} \cdot \hat{P}
$$




$$
\alpha_{i}^{\mu} \equiv \beta_{i} \gamma_{i \perp}^{\mu}=2 \theta_{i \perp}^{\mu} \theta_{i} \cdot \hat{P}
$$

and

$$
\Sigma_{i}^{\mu} \equiv \theta_{\perp i}^{\mu}
$$

in which the subscript $i$ is the particle label. In the c.m. system, $\alpha$ and $\Sigma$ have no time component. In a general frame their components parallel to the total four-momentum $P$ are zero. Using these, we obtain

$$
\begin{aligned}
\theta_{\perp i}^{\mu} & =-i \sqrt{\frac{1}{2}} \beta_{i} \Sigma_{i}^{\mu} \\
\theta_{i} \cdot \hat{P} & =i \sqrt{\frac{1}{2}} \beta_{i} \gamma_{5 i} \\
\theta_{5 i} & =i \sqrt{\frac{1}{2}} \gamma_{5 i} .
\end{aligned}
$$

The two-body Dirac equations Eqs. (2.18a-b) then take the form

$\mathcal{S}_{1} \psi=\left(-G \beta_{1} \Sigma_{1} \cdot \mathcal{P}_{2}+E_{1} \beta_{1} \gamma_{51}+M_{1} \gamma_{51}+G \frac{i}{2} \Sigma_{2} \cdot \partial\left(J \beta_{1}-L \beta_{2}\right) \gamma_{51} \gamma_{52}\right) \psi=0$ $\mathcal{S}_{2} \psi=\left(G \beta_{2} \Sigma_{2} \cdot \mathcal{P}_{1}+E_{2} \beta_{2} \gamma_{52}+M_{2} \gamma_{52}-G \frac{i}{2} \Sigma_{1} \cdot \partial\left(J \beta_{2}-L \beta_{1}\right) \gamma_{51^{\prime}} \gamma_{52}\right) \psi=0$ in which

$$
\mathcal{P}_{i} \equiv p-\frac{i}{2} \Sigma_{i} \cdot \partial \ln G \Sigma_{i}
$$

Thus, $\mathcal{S}_{1}^{2} \psi=0$ becomes

$\left\{\left(G \Sigma_{1} \cdot \mathcal{P}_{2}\right)^{2}-\left[G \beta_{1} \Sigma_{1} \cdot \mathcal{P}_{2}, E_{1} \beta_{1}+M_{1}\right] \gamma_{51}-E_{1}^{2}+M_{1}^{2}+G^{2} \frac{1}{4}(\partial(J-L))^{2}\right.$
$\left.-\left[G \beta_{1} \Sigma_{1} \cdot \mathcal{P}_{2}, G \frac{i}{2} \Sigma_{2} \cdot \partial\left(J \beta_{1}-L \beta_{2}\right)\right] \gamma_{51} \gamma_{52}-i G\left(E_{1} \partial J+\beta_{1} \beta_{2} M_{1} \partial L\right) \cdot \check{\Sigma}_{2} \gamma_{52}\right\} \psi=0$. 
Now we substitute the Dirac equations (4.7a-b) in the forms

$$
\begin{aligned}
& \gamma_{51} \psi=\frac{1}{\left(E_{1} \beta_{1}+M_{1}\right)} G\left[\beta_{1} \Sigma_{1} \cdot \mathcal{P}_{2}-\frac{i}{2} \partial\left(J \beta_{1}-L \beta_{2}\right) \cdot \Sigma_{2} \gamma_{51} \gamma_{52}\right] \psi, \\
& \gamma_{52} \psi=-\frac{1}{\left(E_{2} \beta_{2}+M_{2}\right)} G\left[\beta_{2} \Sigma_{2} \cdot \mathcal{P}_{1}-\frac{i}{2} \partial\left(J \beta_{2}-L \beta_{1}\right) \cdot \Sigma_{1} \gamma_{51} \gamma_{52}\right] \psi,
\end{aligned}
$$

into the respective singly odd parts of (4.10) (those parts that contain only one $\gamma_{5 i}$ factor) and evaluate the commutator appearing in one of the singly odd terms. This yields a single second order Schrödinger-like equation ${ }^{4-5}$

$$
\begin{gathered}
\left(\left(G \Sigma_{1} \cdot \mathcal{P}_{2}\right)^{2}-E_{1}^{2}+M_{1}^{2}+G^{2} \frac{1}{4}(\partial(J-L))^{2}\right. \\
+i G \Sigma_{1} \cdot \partial \ln \left(E_{1} \beta_{1}+M_{1}\right)\left(G \Sigma_{1} \cdot \mathcal{P}_{2}\right)+i G \Sigma_{2} \cdot \partial \ln \left(E_{2} \beta_{2}+M_{2}\right)\left(G \Sigma_{2} \cdot \mathcal{P}_{1}\right) \\
+\left[-\left[G \Sigma_{1} \cdot \mathcal{P}_{2}, G \frac{i}{2} \Sigma_{2} \cdot \partial\left(J-L \beta_{1} \beta_{2}\right)\right]+\frac{1}{2} G^{2} \Sigma_{1} \cdot \partial \ln \left(E_{1} \beta_{1}+M_{1}\right) \partial\left(J-L \beta_{1} \beta_{2}\right) \cdot \Sigma_{2}\right. \\
\left.\left.+\frac{1}{2} G^{2} \Sigma_{2} \cdot \partial \ln \left(E_{2} \beta_{2}+M_{2}\right) \partial\left(J-L \beta_{1} \beta_{2}\right) \cdot \Sigma_{1}\right] \gamma_{51} \gamma_{52}\right) \psi=0 .
\end{gathered}
$$

The sixteen component Dirac spinor in (4.12) we write as

$$
\begin{gathered}
\psi=\psi_{1}\left(\begin{array}{l}
1 \\
0
\end{array}\right)_{1}\left(\begin{array}{l}
1 \\
0
\end{array}\right)_{2}+\psi_{2}\left(\begin{array}{l}
0 \\
1
\end{array}\right)_{1}\left(\begin{array}{l}
1 \\
0
\end{array}\right)_{2}+\psi_{3}\left(\begin{array}{l}
1 \\
0
\end{array}\right)_{1}\left(\begin{array}{l}
0 \\
1
\end{array}\right)_{2}+\psi_{4}\left(\begin{array}{l}
0 \\
1
\end{array}\right)_{1}\left(\begin{array}{l}
0 \\
1
\end{array}\right)_{2} \\
\equiv \sum_{\kappa \lambda} \psi_{\kappa \lambda} \eta_{\kappa}^{(1)} \eta_{\lambda}^{(2)}
\end{gathered}
$$

or simply

$$
\psi^{\prime}=\left[\begin{array}{l}
\psi_{1} \\
\psi_{2} \\
\psi_{3} \\
\psi_{4}
\end{array}\right]
$$

where $\psi_{i}$ are four-component spinors (see Appendix B for the convention we use in defining the $16 \times 16$ gamma matrices for the product space). 
Note that since there are no terms in Eq.(4.12) that contain an odd number of $\gamma_{5}$ matrices, the upper-upper components couple only to the lower-lower components. Eq.(4.12) also couples the lower-upper to the upper-lower components but we will not need the resulting equations since Eqs.(4.11a-b) determine these components in terms of the upperupper and lower-lower components.

Even though in principle the squaring procedure used to construct these Schrödinger-like equations could introduce spurious solutions, it turns out that the equations we obtain by this procedure are identical to those Schrödinger-like equations obtained by simply manipulating the Dirac equations without squaring them ${ }^{47}$. Consider as an illustration the case of a single particle under combined scalar and time-like vector interactions. The one-body Dirac equation can be written in the form

$$
\mathcal{S} \psi=\left(-\beta \vec{\Sigma} \cdot \vec{p}+E \beta \gamma_{5}+M \gamma_{5}\right) \psi=0
$$

in which $E=\epsilon-\mathcal{V}, M=m+S$. In the Dirac representation, in which $\vec{\Sigma}$ and $\beta$ are block diagonal, $\gamma_{5}=\left(\begin{array}{ll}0 & 1 \\ 1 & 0\end{array}\right)$, and

$$
\psi=\left[\begin{array}{l}
\psi_{1} \\
\psi_{2}
\end{array}\right]
$$

Eq.(4.14) becomes

$$
(-\vec{\sigma} \cdot \vec{p}) \psi_{1}+(E+M) \psi_{2}=0
$$

and

$$
(\vec{\sigma} \cdot \vec{p}) \psi_{2}-(E-M) \psi_{1}=0
$$


By substituting $\left.\psi_{2}=(E+M)^{-1}(\vec{\sigma} \cdot \vec{p})\right) \psi_{1}$ into the first equation one obtains

$$
\left(\vec{p}^{2}-E^{2}+M^{2}+i \vec{\sigma} \cdot \vec{\nabla} \ln (E+M) \vec{\sigma} \cdot \vec{p}\right) \psi_{1}=0
$$

and similarly

$$
\left(\vec{p}^{2}-E^{2}+M^{2}-i \vec{\sigma} \cdot \vec{\nabla} \ln (E-M) \vec{\sigma} \cdot \vec{p}\right) \psi_{2}=0
$$

When one solves Eq.(4.17a) for $\psi_{1}$ and uses Eq.(4.16a) to determine the corresponding $\psi_{2}$, one obtains the same four-component wave function that arises from direct solution of Eqs.(4.16a-b). Of course to complete the solution we must start from the solution $\psi_{2}$ of Eq. $(4.17 \mathrm{~b})$ and use Eq.(4.16b) to determine the corresponding $\psi_{1}$. An alternative derivation of $(4.17 \mathrm{a}-\mathrm{b})$ that is analogous to that which we gave for our two-body Dirac equations starts with $\mathcal{S}^{2} \psi=0$, i.e.

$$
\left((\vec{\Sigma} \cdot \vec{p})^{2}-[\beta \vec{\Sigma} \cdot \vec{p}, E \beta+M] \gamma_{5}-E^{2}+M^{2}\right) \psi=0
$$

By using the Dirac equation (4.14) in the form

$$
\gamma_{5} \psi=\frac{1}{(E \beta+M)}(\beta \vec{\Sigma} \cdot \vec{p}) \psi
$$

we can substitute for the Dirac wave function in the $\gamma_{5}$ term in (4.18). Evaluation of the commutator leads to

$$
\left(\vec{p}^{2}-E^{2}+M^{2}+i \vec{\Sigma} \cdot \partial \ln (E \beta+M) \vec{\Sigma} \cdot \vec{p}\right) \psi=0
$$

In the Dirac representation, this procedure does lead to the two uncoupled, two-component equations (4.17a-b). Thus, in spite of the squaring 
of the Dirac operator, this procedure leads to the same set of equations as obtained through decoupling the two component Dirac equations by substituting one into the other. It is gratifying that the two approaches lead to same equations. If they were not equivalent, then the difference between the equations produced by the two approaches would lead to a new constraint not appearing in the original one-body Dirac equation.

In appendix $\mathrm{B}$, where we perform similar manipulations for the twobody Dirac equations, we obtain (without squaring) the upper-upper component of (4.12) (the other components could be obtained by an analogous procedure), thus showing the equivalence of the two approaches (not demonstrated in Refs.(4-5)). Comparison of the two procedures leads to no further constraints beyond the original two-body Dirac equations. Just as the solutions of the one-body equation Eq.(4.14) are obtained from the $\psi_{1}$ (or $\psi_{2}$ ) of Eq.(4.17) and $\psi_{2}$ (or $\psi_{1}$ ) of Eq.(4.19), so one could construct the full sixteen component solutions of Eqs.(4.7ab) by solving the upper-upper and lower-lower portions of Eq.(4.12) for $\psi_{1}$ and $\psi_{4}$ and then using Eq.(4.11a-b) to obtain $\psi_{2}$ and $\psi_{3}$. Having constructed the full solutions to the coupled sixteen component Dirac equations we could in principle use them in conjunction with the inner product $^{36}$ derived from these Dirac equations for the sixteen component wave functions and apply them to the computation of decay and other current matrix elements.

In appendix B we also perform simplifying Pauli matrix algebra on the coupled upper-upper and lower-lower components of Eq.(4.12). We find that the upper-upper component of (4.12) becomes

$$
\left(p^{2}+2 m_{w} S+S^{2}+2 \epsilon_{w} \mathcal{A}-\mathcal{A}^{2}+2 \epsilon_{w} \mathcal{V}-\mathcal{V}^{2}\right.
$$




$$
\begin{gathered}
\left.+\frac{1}{4}(\partial J-\partial L)^{2}+i \ln ^{\prime} \chi_{1} \chi_{2} \hat{r} \cdot p-\frac{1}{2} \partial^{2} \mathcal{G}+\frac{3}{4}\left(\mathcal{G}^{\prime}\right)^{2}+\frac{1}{2} \ln ^{\prime} \chi_{1} \chi_{2} \mathcal{G}^{\prime}\right) \\
-\frac{\ln ^{\prime} \chi_{1}}{r} L \cdot \sigma_{1}-\frac{l^{\prime} \chi_{2}}{r} L \cdot \sigma_{2} \\
+\left[\frac{1}{3} \partial^{2} \mathcal{G}-\frac{1}{2}\left(\mathcal{G}^{\prime}\right)^{2}-\frac{1}{3} l^{\prime} \chi_{1} \chi_{2} \mathcal{G}^{\prime}\right] \sigma_{1} \cdot \sigma_{2} \\
\left.\left[-\frac{1}{6}\left(\mathcal{G}^{\prime \prime}-\frac{\mathcal{G}^{\prime}}{r}\right)+\frac{1}{6} l^{\prime} \chi_{1} \chi_{2} \mathcal{G}^{\prime}\right] S_{T}\right) \psi_{1} \\
+\left(\left[+\frac{1}{6} l^{\prime} \chi_{1} \chi_{2}(J-L)^{\prime}+\frac{1}{2} \mathcal{G}^{\prime}(J-L)^{\prime}-\frac{1}{6} \partial^{2}(J-L)\right] \sigma_{1} \cdot \sigma_{2}\right. \\
\left.\left[+\frac{1}{6} \ln ^{\prime} \chi_{1} \chi_{2}(J-L)^{\prime}-\frac{1}{6}\left((J-L)^{\prime \prime}-\frac{(J-L)^{\prime}}{r}\right)\right] S_{T}\right) \psi_{4}=b^{2}(w) \psi_{1}
\end{gathered}
$$

in which $\chi_{i}=\left(E_{i}+M_{i}\right) / G$. This couples to the lower-lower component of (4.12) which becomes

$$
\begin{gathered}
\left(p^{2}+2 m_{w} S+S^{2}+2 \epsilon_{w} \mathcal{A}-\mathcal{A}^{2}+2 \epsilon_{w} \mathcal{V}-\mathcal{V}^{2}\right. \\
\left.+\frac{1}{4}(\partial J-\partial L)^{2}+i \ln ^{\prime} \bar{\chi}_{1} \bar{\chi}_{2} \hat{r} \cdot p-\frac{1}{2} \partial^{2} \mathcal{G}+\frac{3}{4}\left(\mathcal{G}^{\prime}\right)^{2}+\frac{1}{2} \ln ^{\prime} \bar{\chi}_{1} \bar{\chi}_{2} \mathcal{G}^{\prime}\right) \\
-\frac{\ln ^{\prime} \bar{\chi}_{1}}{r} L \cdot \sigma_{1}-\frac{\ln ^{\prime} \bar{\chi}_{2}}{r} L \cdot \sigma_{2} \\
+\left[\frac{1}{3} \partial^{2} \mathcal{G}-\frac{1}{2}\left(\mathcal{G}^{\prime}\right)^{2}-\frac{1}{3} \ln ^{\prime} \bar{\chi}_{1} \bar{\chi}_{2} \mathcal{G}^{\prime}\right] \sigma_{1} \cdot \sigma_{2} \\
\left.\left[-\frac{1}{6}\left(\mathcal{G}^{\prime \prime}-\frac{\mathcal{G}^{\prime}}{r}\right)+\frac{1}{6} \ln ^{\prime} \bar{\chi}_{1} \bar{\chi}_{2} \mathcal{G}^{\prime}\right] S_{T}\right) \psi_{4} \\
+\left(\left[+\frac{1}{6} \ln ^{\prime} \bar{\chi}_{1} \bar{\chi}_{2}(J-L)^{\prime}+\frac{1}{2} \mathcal{G}^{\prime}(J-L)^{\prime}-\frac{1}{6} \partial^{2}(J-L)\right] \sigma_{1} \cdot \sigma_{2}\right. \\
\left.\left[+\frac{1}{6} \ln ^{\prime} \bar{\chi}_{1} \bar{\chi}_{2}(J-L)^{\prime}-\frac{1}{6}\left((J-L)^{\prime \prime}-\frac{(J-L)^{\prime}}{r}\right)\right] S_{T}\right) \psi_{1}=b^{2}(w) \psi_{4}(4.21 b
\end{gathered}
$$

in which $\bar{\chi}_{i}=\left(E_{i}-M_{i}\right) / G$. Eqs.(4.21a-b) are the two coupled eightcomponent Schrödinger-like equations that we shall use in Secs. V and 
VI for our bound state calculations. The same two equations would have been obtained if we had started with $\mathcal{S}_{2}^{2}$ instead of $\mathcal{S}_{1}^{2}$ since their difference is $\sim \hat{P} \cdot p \psi=0$. Although not given here, the corresponding equations that couple the upper-lower and lower-upper components can be combined with Eqs.(4.21a-b) into the general form

$$
\begin{gathered}
\left(p^{2}+\Phi_{w}\right) \psi \\
=\left(p^{2}+\Phi_{\text {S.I. }}+\Phi_{D .}+\Phi_{\text {S.O. }}+\Phi_{\text {S.S. }}+\Phi_{T .}+\Phi_{D . O .} \gamma_{51} \gamma_{52}\right) \psi=b^{2}(w) \psi
\end{gathered}
$$

in which $\psi$ is the full sixteen component wave function. The forms of the parts of the quasipotential are given as in Eq.(4.21a) but with $\chi_{i}=E_{i}+\beta_{i} M_{i} . \Phi_{\text {S.I. }}$ is the spin-independent minimal (Todorov) portion of the quasipotential, $\Phi_{D}$. denotes the Darwin interactions (the second line of Eq.(4.21a-b)), $\Phi_{S . O}$. the spin-orbit, $\Phi_{S . S .}$ the spin-spin, and $\Phi_{T}$. the tensor portions of the quasipotential. $\Phi_{D . O}$. is the doubly odd part which couples the upper-upper and lower-lower, or the upper-lower and lower-upper portions of the wave function ${ }^{48}$. As discussed in appendix $\mathrm{D}$ a further decoupling of these equations can occur for special angular momentum states. 
V Perturbative Treatment of the Two-Body Dirac Equations

Here we examine the bound state energies produced by our constraint equations $(4.21 \mathrm{a}-\mathrm{b})$ in the weak-potential limit in order ro obtain the energy spectra of our relativistic two-body system analytically through order $\alpha^{4}$ by means of perturbation theory. For the case of the purely electromagnetic interaction $(\mathcal{A}=-\alpha / r, \mathcal{V}=S=0)$, we compare the spectra with those obtained in perturbative QED from the FermiBreit reduction of the Bethe-Salpeter equation ${ }^{31}$. Since scalar and purely time-like interactions were also important in the meson spectroscopy work of Refs.1-2, we will also compute the energy spectra analytically by using perturbation theory for the scalar and time-like four vector interactions $(S=-\alpha / r, \mathcal{V}=0=\mathcal{A}$ for the scalar and $\mathcal{V}=-\alpha / r, S=0=\mathcal{A}$ for the time-like four vector interaction) through order $\alpha^{4}$. In section VI we compare the perturbative eigenvalues with those obtained from a nonperturbative numerical solution of the unapproximated constraint equations, Eqs.(4.21a-b). The comparison with the standard spectral results from the Bethe-Salpeter equation for the case of the purely electromagneticlike interactions will provide a critical test at both the perturbative and nonperturbative levels of the capability of the two-body Dirac equations of constraint dynamics to generate accurate spin-dependent as well as spin- independent relativistic recoil corrections for QED, from a static input potential $\mathcal{A}(r)$. The comparisons between the perturbative and nonperturbative solutions of the two body Dirac equations for the scalar and time-like vector interactions are also important since they will reveal whether or not the unapproximated equations yield the perturbative rel- 
ativistic (primarily short-distance) corrections of lowest order for those interactions. For example, in the case of a purely scalar interaction, there should be no hyperfine splitting through order $\alpha^{4}$. A glance at Eqs. (4.21a-b) shows that these equations contain terms (e.g. $\left.\partial^{2} L \sigma_{1} \cdot \sigma_{2}\right)$ that could contribute to such a splitting. If a numerical treatment of the unapproximated equations with these interactions showed a larger hyperfine splitting than that predicted from a perturbative treatment of the equations, then these equations would not be trustworthy relativistic equations for meson spectroscopy calculations in which more general effective scalar and time-like four vector interactions are used.

\section{A. General Equations}

In order to perturbatively evaluate the energy eigenvalues we first obtain the weak-potential form that we will use from Eq.(4.21a) for the upper-upper component $\psi_{1}$ of the wave function, coupled to $\psi_{4}$. In the weak-potential limit we ignore the coupling to the lower-lower wave function $\psi_{4}$. Furthermore in the weak potential limit, in terms of the total mass $M=m_{1}+m_{2}$ we make the replacements (see Eqs.(2.10-2.11) and (3.48))

$$
\begin{gathered}
\mathcal{G} \approx \frac{\mathcal{A}}{M} \\
L \approx \frac{S}{M} \\
J \approx-\frac{\mathcal{A}+\mathcal{V}}{M}, \\
\chi_{1} \approx 2 m_{1}\left(1-\frac{\mathcal{A}}{M}\right)+m_{2} \frac{S-\mathcal{A}-\mathcal{V}}{M}
\end{gathered}
$$


and

$$
\chi_{2} \approx 2 m_{2}\left(1-\frac{\mathcal{A}}{M}\right)+m_{1} \frac{S-\mathcal{A}-\mathcal{V}}{M}
$$

In this approximation Eq.(4.21a) simplifies to

$$
\begin{gathered}
\left\{p^{2}+2 m_{w} S+S^{2}+2 \epsilon_{w} \mathcal{A}-\mathcal{A}^{2}+2 \epsilon_{w} \mathcal{V}-\mathcal{V}^{2}\right. \\
-\frac{1}{2} \partial_{\perp}^{2} \frac{\mathcal{A}}{M}+i \frac{\mathcal{A}^{\prime}(-1-M / 2 \mu)+\left(S^{\prime}-\mathcal{V}^{\prime}\right)(-1+M / 2 \mu)}{M} \hat{r} \cdot p \\
+\left[\frac{\mathcal{A}^{\prime}}{M}-\frac{1}{2} \frac{m_{2}}{m_{1}} \frac{(S-\mathcal{A}-\mathcal{V})^{\prime}}{M}\right] \frac{L \cdot \sigma_{1}}{r}+\left[\frac{\mathcal{A}^{\prime}}{M}-\frac{1}{2} \frac{m_{1}}{m_{2}} \frac{(S-\mathcal{A}-\mathcal{V})^{\prime}}{M}\right] \frac{L \cdot \sigma_{2}}{r} \\
\left.+\frac{1}{3} \frac{\partial_{\perp}^{2} \mathcal{A}}{M} \sigma_{1} \cdot \sigma_{2}+\left[-\frac{1}{6} \frac{\left(\mathcal{A}^{\prime \prime}-\frac{\mathcal{A}^{\prime}}{r}\right)}{M}\right] S_{T}\right\} \psi_{1} \\
=b^{2}(w) \psi_{1}
\end{gathered}
$$

in which $\mu=m_{1} m_{2} / M$.

We now specialize to three cases.

(1.) $\mathcal{V}=S=0$ leads to the weak-potential form of the equation containing only an electromagnetic interaction generated by the invariant $\mathcal{A}$,

$$
\begin{gathered}
\left\{p^{2}+2 \epsilon_{w} \mathcal{A}-\mathcal{A}^{2}-\frac{1}{2} \partial_{\perp}^{2} \frac{\mathcal{A}}{M}+i \frac{\mathcal{A}^{\prime}(-1-M / 2 \mu)}{M} \hat{r} \cdot p\right. \\
+\frac{\mathcal{A}^{\prime}}{M}\left(1+\frac{1}{2} \frac{m_{2}}{m_{1}}\right) \frac{L \cdot \sigma_{1}}{r}+\frac{\mathcal{A}^{\prime}}{M}\left(1+\frac{1}{2} \frac{m_{1}}{m_{2}}\right) \frac{L \cdot \sigma_{2}}{r} \\
\left.+\frac{1}{3} \frac{\partial_{\perp}^{2} \mathcal{A}}{M} \sigma_{1} \cdot \sigma_{2}-\frac{1}{6} \frac{\left(\mathcal{A}^{\prime \prime}-\frac{\mathcal{A}^{\prime}}{r}\right)}{M} S_{T}\right\} \psi_{1} \\
=b^{2}(w) \psi_{1} .
\end{gathered}
$$

For lowest order electrodynamics we found in section III that the quasipotential reduction of $\mathrm{QED}$ led to

$$
\Delta^{(1)}=\theta_{51} \theta_{52} \gamma_{1}^{\mu} \gamma_{2 \mu} \frac{e_{1} e_{2}}{4 \pi w\left|x_{\perp}\right|}=\frac{e_{1} e_{2}}{4 \pi w\left|x_{\perp}\right|} \theta_{1} \cdot \theta_{2}
$$


This corresponds to $\mathcal{G}^{(1)}=-J^{(1)}=-\frac{\alpha}{w r}$, and $L^{(1)}=0$ where $\alpha=$ $-e_{1} e_{2} / 4 \pi$. To the order we are considering, we can set $w=M$ so that (3.48c) implies $\mathcal{A}=-\alpha / r$. In terms of the dimensionless Coulomb variable $\vec{x}={ }_{w} \alpha \vec{r}$, Eq. (5.7) becomes ${ }^{49}$

$$
\begin{gathered}
\left\{\left(-\frac{1}{x} \frac{d^{2}}{d x^{2}} x+\frac{\vec{L}^{2}}{x^{2}}-\frac{2}{x}\right)+\alpha^{2}\left\{-\frac{1}{x^{2}}-\left(\frac{\mu}{M}+\frac{1}{2}\right) \frac{1}{x^{2}} \frac{d}{d x}+\pi \delta^{3}(\vec{x}) \frac{\mu}{M}\left(-2+\frac{4}{3} \vec{\sigma}_{1} \cdot \vec{\sigma}_{2}\right)\right.\right. \\
+\frac{1}{x^{3}} \vec{L} \cdot\left(\vec{\sigma}_{1}+\vec{\sigma}_{2}\right)\left(\frac{\mu}{2 M}+\frac{1}{4}\right)+\frac{1}{x^{3}} \vec{L} \cdot\left(\vec{\sigma}_{1}-\vec{\sigma}_{2}\right) \frac{m_{2}-m_{1}}{4 M} \\
\left.+\frac{\mu}{2 M x^{3}} S_{T}\right\} \psi_{1}=-\lambda^{2} \psi_{1}
\end{gathered}
$$

in which $\lambda^{2}=-b^{2}(w) /\left(\epsilon_{w} \alpha\right)^{2}$.

(2.) $\mathcal{A}=\mathcal{V}=0$ leads to the weak-potential form of the equation, containing only a scalar interaction generated by the invariant $S$

$$
\begin{gathered}
\left\{p^{2}+2 m_{w} S+S^{2}+\frac{S^{\prime}(-1+M / 2 \mu)}{M} \hat{r} \cdot p\right. \\
\left.-\frac{1}{2} \frac{m_{2}}{m_{1}} \frac{S^{\prime}}{M} \frac{L \cdot \sigma_{1}}{r}-\frac{1}{2} \frac{m_{1}}{m_{2}} \frac{S^{\prime}}{M} \frac{L \cdot \sigma_{2}}{r}\right\} \psi_{1}=b^{2}(w) \psi_{1} .
\end{gathered}
$$

For our model scalar interaction, the analog for scalar field theory of the quasipotential reduction of section III leads to

$$
\Delta^{(1)}=\theta_{51} \theta_{52} \frac{g_{1} g_{2}}{4 \pi w\left|x_{\perp}\right|}
$$

This corresponds to $\mathcal{G}^{(1)}=J^{(1)}=0$, and $L^{(1)}=-\frac{\alpha}{w r}$, where $\alpha=$ $g_{1} g_{2} / 4 \pi$. To the order we are considering we can set $w=M$, so that Eq.(3.48a) implies $S=-\alpha / r$. In terms of the dimensionless variable $\vec{x}=m_{w} \alpha \vec{r}$, Eq.(5.10) becomes

$$
\left\{\left(-\frac{1}{x} \frac{d^{2}}{d x^{2}} x+\frac{\vec{L}^{2}}{x^{2}}-\frac{2}{x}\right)+\alpha^{2}\left\{\frac{1}{x^{2}}-\left(\frac{\mu}{M}-\frac{1}{2}\right) \frac{1}{x^{2}} \frac{d}{d x}\right.\right.
$$




$$
\left.-\frac{1}{4 x^{3}} \vec{L} \cdot\left(\vec{\sigma}_{1}+\vec{\sigma}_{2}\right)-\frac{1}{x^{3}} \vec{L} \cdot\left(\vec{\sigma}_{1}-\vec{\sigma}_{2}\right) \frac{m_{2}-m_{1}}{4 M}\right\} \psi_{1}=-\lambda^{2} \psi_{1},
$$

in which $\lambda^{2}=-b^{2}(w) /\left(m_{w} \alpha\right)^{2}$.

3. $\mathcal{A}=S=0$ leads to the weak-potential form of the equation containing only time-like vector interactions generated by the invariant $\mathcal{V}$

$$
\begin{gathered}
\left\{p^{2}+2 \epsilon_{w} \mathcal{V}-\mathcal{V}^{2}-i \frac{\mathcal{V}^{\prime}(-1+M / 2 \mu)}{r} \hat{r} \cdot p\right. \\
\left.+\frac{1}{2} \frac{m_{2}}{m_{1}} \frac{\mathcal{V}^{\prime}}{M} \frac{L \cdot \sigma_{1}}{r}+\frac{1}{2} \frac{m_{1}}{m_{2}} \frac{\mathcal{V}^{\prime}}{M} \frac{L \cdot \sigma_{2}}{r}\right\} \psi_{1}=b^{2}(w) \psi_{1}
\end{gathered}
$$

If we had separated the time-like and space-like parts of the electromagnetic interaction Eq.(5.8) and retained only the time-like part we would have obtained

$$
\Delta^{(1)}=\theta_{1} \cdot \hat{P} \theta_{2} \cdot \hat{P} \frac{e_{1} e_{2}}{4 \pi w\left|x_{\perp}\right|}
$$

This choice for our model time-like vector interaction corresponds to $\mathcal{G}^{(1)}=L^{(1)}=0$ and $J^{(1)}=-\frac{\alpha}{w r}$, where $\alpha=-e_{1} e_{2} / 4 \pi$. Again we can set $w=M$ so that Eq.(3.48b) implies $\mathcal{V}=-\alpha / r$. In terms of the dimensionless Coulomb variable $\vec{x}=\epsilon_{w} \alpha \vec{r}$, Eq.(5.13) becomes

$$
\begin{array}{r}
\left\{\left(-\frac{1}{x} \frac{d^{2}}{d x^{2}} x+\frac{\vec{L}^{2}}{x^{2}}-\frac{2}{x}\right)+\alpha^{2}\left\{-\frac{1}{x^{2}}+\left(\frac{\mu}{M}-\frac{1}{2}\right) \frac{1}{x^{2}} \frac{d}{d x}\right.\right. \\
\left.+\frac{1}{4 x^{3}} \vec{L} \cdot\left(\vec{\sigma}_{1}+\vec{\sigma}_{2}\right)+\frac{1}{x^{3}} \vec{L} \cdot\left(\vec{\sigma}_{1}-\vec{\sigma}_{2}\right) \frac{m_{2}-m_{1}}{4 M}\right\} \psi_{1}=-\lambda^{2} \psi_{1},
\end{array}
$$

in which $\lambda^{2}=-b^{2}(w) /\left(\epsilon_{w} \alpha\right)^{2}$.

In all three cases we have, to the lowest order, $-\lambda^{2}=-1 / n^{2}$ with the unperturbed wave function given by

$$
\psi_{n l s j m}=R_{n l} \mathcal{Y}_{l s j m}
$$


in which

$$
R_{n l}=\frac{u_{n l}}{x}=\left[\left(\frac{2}{n}\right)^{3} \frac{(n-l-1) !}{2 n(n+l) !}\right]^{\frac{1}{2}} e^{-x / n}\left(\frac{2 x}{n}\right)^{l} L_{n-l-1}^{2 l+1}\left(\frac{2 x}{n}\right)
$$

and $\mathcal{Y}_{l s j m}$ is the total angular momentum eigenfunction. The quantum numbers $j l s m$ given here refer to those of the upper-upper component of the wave function. In general, $j, m$, and parity are the only good quantum numbers for the wave function as a whole. (For the equal mass case, charge parity is also a valid quantum number). The $\alpha^{2}$ terms will be treated as first order perturbations; they eliminate some of the $2 n^{2}$ fold degeneracy of the unperturbed state. For the $n=1$ states, the twofold degeneracy between the ${ }^{1} S_{0}$ and ${ }^{3} S_{1}$ states is removed only by the electromagnetic interaction since the scalar and time-like vector interactions do not have any spin-spin terms to this order. We note also that the tensor term does not produce any first-order shift between singlet and triplet ground states, since $l=0$.

For the $n=2$ level, every $\alpha^{2}$ term contributes to the removal of the degeneracy between the $l=0$ and $l=1$ states $\left({ }^{1} S_{0},{ }^{3} S_{1},{ }^{1} P_{1},{ }^{3} P_{0},{ }^{3} P_{1},{ }^{3} P_{2}\right)$. Again, only the spin-spin term in the electromagnetic interaction can remove the spin degeneracy in the $l=0$ states to this order. For the $l=1$ states, the spin-orbit interaction $\left(\vec{L} \cdot\left(\vec{\sigma}_{1}+\vec{\sigma}_{2}\right)\right)$ and also the tensor term split them into four levels. Furthermore, for unequal masses the spin degeneracy is removed between the ${ }^{1} P_{1}$ and ${ }^{3} P_{1}$ states by a diagonalization of the spin-orbit difference term $\left(\vec{L} \cdot\left(\ddot{\sigma}_{1}-\vec{\sigma}_{2}\right)\right)$. This spin-mixing term is crucial in merging the four $P$ states into two $P$ states in the limit that one of the particles becomes very heavy (see spectral results below). The tensor term does not mix spin, but mixes the $l=0$ and 
the $l=2$ states. However, as in the case of the ground state, this mixing will not produce any first order perturbative shifts in the spectrum, since the lowest $l=2$ state has $n=3$ and is not degenerate with the $n=2$ (or 1 ), $l=0$ state. The same comment applies to the $l=1$ and $l=3$ mixing.

For the levels with $n \geq 3$ one might expect that the tensor force would provide an additional first order splitting beyond that appearing for the $n=2$ level. However, there is no such additional splitting from the $l$-mixing since the radial matrix element $\left\langle n l\left|\frac{1}{x^{3}}\right| n l^{\prime}\right\rangle$ vanishes for $\left|l-l^{\prime}\right|=2$ (see Appendix C).

\section{B. General Spectra}

The results of the perturbative calculations are summarized below. We present details in Appendix C.

(1.) For the electromagnetic interactions, the binding energy through order $\alpha^{4}$ is

$$
\epsilon_{B} \equiv w-M=-\frac{\mu \alpha^{2}}{2 n^{2}}+\frac{\mu \alpha^{4}}{2 n^{3}} \eta+\frac{\mu \alpha^{4}}{8 n^{4}}\left(3-\frac{\mu}{M}\right),
$$

in which

$$
\begin{aligned}
\eta=-\frac{2}{2 l+1}+\delta_{l 0}+ & \frac{(j(j+1)-l(l+1)-2)\left(\frac{\mu}{M}+\frac{1}{2}\right)+\kappa_{l j} \frac{\mu}{M}}{(2 l+1) l(l+1)}\left(1-\delta_{l 0}\right)\left(1-\delta_{s 0}\right) \\
& +\frac{8 \mu}{3 M} \delta_{l 0}\left[s(s+1)-\frac{3}{2}\right], j \neq l \geq 1
\end{aligned}
$$

with

$$
\kappa_{l j} \equiv-\frac{2 l}{2 l+3} \delta_{j l+1}+2 \delta_{j l}-\frac{2(l+1)}{(2 l-1)} \delta_{j l-1},
$$


for all states except $j=l \geq 1$. For the $j=l \geq 1$ states, the spin mixing term produces the split spectrum

$$
\epsilon_{B}=w-M=-\frac{\mu \alpha^{2}}{2 n^{2}}+\frac{\mu \alpha^{4}}{2 n^{3}} \eta_{ \pm}+\frac{\mu \alpha^{4}}{8 n^{4}}\left(3-\frac{\mu}{M}\right), j=l \geq 1
$$

in which

$$
\eta_{ \pm}=a+c \pm \sqrt{(a-c)^{2}+4 b^{2}}
$$

with

$$
\begin{gathered}
a=-\frac{2}{2 l+1}, \\
b=\frac{1}{(2 l+1) \sqrt{(l(l+1)}} \frac{m_{2}-m_{1}}{M},
\end{gathered}
$$

and

$$
c=-\frac{2}{2 l+1}-\frac{1}{(2 l+1) l(l+1)} .
$$

These are the standard results ${ }^{50}$ of Ref.(31).

(2.) For the scalar interaction, the binding energy through order $\alpha^{4}$ is

$$
\epsilon_{B} \equiv w-M=-\frac{\mu \alpha^{2}}{2 n^{2}}+\frac{\mu \alpha^{4}}{2 n^{3}} \eta+\frac{\mu \alpha^{4}}{8 n^{4}}\left(-1-\frac{\mu}{M}\right),
$$

in which

$$
\begin{gathered}
\eta=\frac{2}{2 l+1}-\delta_{l 0}\left(1-\frac{2 \mu}{M}\right) \\
+\frac{(j(j+1)-l(l+1)-2)\left(\frac{\mu}{M}-\frac{1}{2}\right)}{(2 l+1) l(l+1)}\left(1-\delta_{l 0}\right)\left(1-\delta_{s 0}\right), j \neq l \geq 1,
\end{gathered}
$$

for all states except the $j=l \geq 1$. For the $j=l \geq 1$ states, the spin mixing term produces the split spectrum

$$
\epsilon_{B}=w-M=-\frac{\mu \alpha^{2}}{2 n^{2}}+\frac{\mu \alpha^{4}}{2 n^{3}} \eta_{ \pm}+\frac{\mu \alpha^{4}}{8 n^{4}}\left(-1-\frac{\mu}{M}\right), j=l \geq 1,
$$


in which

$$
\begin{gathered}
\eta_{ \pm}=a+c \pm \sqrt{(a-c)^{2}+4 b^{2}}, \\
a=\frac{2}{2 l+1}, \\
b=-\frac{1}{(2 l+1) \sqrt{(l(l+1)}} \frac{m_{2}-m_{1}}{M},
\end{gathered}
$$

and

$$
c=\frac{2}{2 l+1}+\frac{1}{(2 l+1) l(l+1)}\left(1-\frac{2 \mu}{M}\right) .
$$

This is the spectrum that would come from the Breit equation if the exchanged photon had spin zero ${ }^{51}$.

(3.) For the time-like vector interaction, the binding energy through order $\alpha^{4}$ is

$$
\epsilon_{B} \equiv w-M=-\frac{\mu \alpha^{2}}{2 n^{2}}+\frac{\mu \alpha^{4}}{2 r^{3}} \eta+\frac{\mu \alpha^{4}}{8 n^{4}}\left(3-\frac{\mu}{M}\right)
$$

in which

$$
\begin{gathered}
\eta=-\frac{2}{2 l+1}+\delta_{l 0}\left(1-\frac{2 \mu}{M}\right) \\
+\frac{(-l(l+1)-2)\left(\frac{\mu}{M}-\frac{1}{2}\right)}{(2 l+1) l(l+1)}\left(1-\delta_{l 0}\right)\left(1-\delta_{s 0}\right), j \neq l \geq 1
\end{gathered}
$$

for all states except the $j=l \geq 1$. For the $j=l \geq 1$ states, the spin mixing term produces the split spectrum

$$
\epsilon_{B}=w-M=-\frac{\mu \alpha^{2}}{2 n^{2}}+\frac{\mu \alpha^{4}}{2 n^{3}} \eta_{ \pm}+\frac{\mu \alpha^{4}}{8 n^{4}}\left(3-\frac{\mu}{M}\right), j=l \geq 1
$$

in which

$$
\begin{gathered}
\eta_{ \pm}=a+c \pm \sqrt{(a-c)^{2}+4 b^{2}} \\
a=-\frac{2}{2 l+1}
\end{gathered}
$$




$$
b=\frac{1}{(2 l+1) \sqrt{(l(l+1)}} \frac{m_{2}-m_{1}}{M}
$$

and

$$
c=-\frac{2}{2 l+1}-\frac{1}{(2 l+1) l(l+1)}\left(1-\frac{2 \mu}{M}\right) .
$$

These spectral results agree with those of QED to lowest order if the effects of the transverse photon are omitted.

\section{One-Body Limit}

In the limit $m_{2} \rightarrow \infty$, all these results should reduce to the corresponding one-body Dirac spectra. In that limit, the electromagnetic and time-like vector results reduce to the common form

$$
\epsilon_{B}=-\frac{m \alpha^{2}}{2 n^{2}}+\frac{m \alpha^{4}}{2 n^{3}} \eta+\frac{3 m \alpha^{4}}{8 n^{4}}
$$

in which

$$
\eta=-\frac{2}{2 l+1}+\delta_{l 0}+\frac{(j(j+1)-l(l+1)-2)}{2(2 l+1) l(l+1)}\left(1-\delta_{l 0}\right),
$$

for $j=0$ and $j=l \pm 1$ states. The binding energies of the $j=l \geq 1$ states, split by the spin mixing term, reduce as $m_{2} \rightarrow \infty$ to

$$
\epsilon_{B}=-\frac{m \alpha^{2}}{2 n^{2}}+\frac{m \alpha^{4}}{2 n^{3}} \eta_{ \pm}+\frac{3 m \alpha^{4}}{8 n^{4}}, \text { for } j=l \geq 1
$$

in which

$$
\begin{gathered}
\eta_{ \pm}=\left(a+c \pm \sqrt{\left.(a-c)^{2}+4 b^{2}\right)}\right. \\
a=-\frac{2}{2 l+1} \\
b=\frac{1}{(2 l+1) \sqrt{(l(l+1)}}
\end{gathered}
$$


and

$$
c=-\frac{2}{2 l+1}-\frac{1}{(2 l+1) l(l+1)} .
$$

For the singlet and triplet $l=0$ states, the binding energies go into a single expression given by

$$
\epsilon_{B}=-\frac{m \alpha^{2}}{2 n^{2}}-\frac{m \alpha^{4}}{n^{3}}\left(\frac{1}{2}-\frac{3}{8 n}\right), \text { for } l=0 .
$$

The binding energies of the $l=j \pm 1 \geq 1$ states become

$$
\epsilon_{B}=-\frac{m \alpha^{2}}{2 n^{2}}-\frac{m \alpha^{4}}{n^{3}}\left(\frac{1}{2 l}-\frac{3}{8 n}\right), \text { for } l=j+1,
$$

and

$$
\epsilon_{B}=-\frac{m \alpha^{2}}{2 n^{2}}-\frac{m \alpha^{4}}{n^{3}}\left(\frac{1}{2 l+2}-\frac{3}{8 n}\right), \text { for } l=j-1,
$$

respectively, and coincide with the spin mixed $l=j \geq 1$ energies.

The exact one-body Dirac spectrum for hydrogen with an infinitely heavy pointlike proton, when expanded out through terms of order $\alpha^{4}$, is

$$
\epsilon_{B}=-\frac{m \alpha^{2}}{2 n^{2}}-\frac{m \alpha^{4}}{n^{3}}\left(\frac{1}{2|k|}-\frac{3}{8 n}\right),
$$

in which $k=l+1$ for $j=l+\frac{1}{2}$ and $k=-l$ for $j=l-\frac{1}{2}$, with $l$ the angular momentum of the large component wave function. In this case Eqs.(5.40)-(5.49), which are the two-body constraint results in the limit $m_{2} \rightarrow \infty$, produce exactly this one-body result.

For scalar interactions in the static limit, our perturbative treatment gives

$$
\epsilon_{B}=-\frac{m \alpha^{2}}{2 n^{2}}+\frac{m \alpha^{4}}{2 n^{3}} \eta-\frac{m \alpha^{4}}{8 n^{4}}
$$


in which

$$
\eta=\frac{2}{2 l+1}-\delta_{l 0}-\frac{(j(j+1)-l(l+1)-2)}{2(2 l+1) l(l+1)}\left(1-\delta_{l 0}\right),
$$

for $j=0$ and $j=l \pm 1$ states. The binding energies of the $j=l \geq 1$ states, which are split by the spin mixing term, are

$$
\epsilon_{B}=-\frac{m \alpha^{2}}{2 n^{2}}+\frac{m \alpha^{4}}{2 n^{3}} \eta_{ \pm}-\frac{m \alpha^{4}}{8 n^{4}}, \text { for } j=l \geq 1,
$$

in which

$$
\begin{gathered}
\eta_{ \pm}=a+c \pm \sqrt{(a-c)^{2}+4 b^{2}} \\
a=\frac{2}{2 l+1} \\
b=-\frac{1}{(2 l+1) \sqrt{(l(l+1)}}
\end{gathered}
$$

and

$$
c=\frac{2}{2 l+1}+\frac{1}{(2 l+1) l(l+1)} .
$$

In this case, the binding energies for the singlet and triplet $l=0$ states become

$$
\epsilon_{B}=-\frac{m \alpha^{2}}{2 n^{2}}-\frac{m \alpha^{4}}{n^{3}}\left(-\frac{1}{2}+\frac{1}{8 n}\right), \text { for } l=0,
$$

while the binding energies for the $l=j \pm 1 \geq 1$ states become

$$
\begin{gathered}
\epsilon_{B}=-\frac{m \alpha^{2}}{2 n^{2}}-\frac{m \alpha^{4}}{n^{3}}\left(-\frac{1}{2 l}+\frac{1}{8 n}\right), \text { for } l=j+1, \\
\epsilon_{B}=-\frac{m \alpha^{2}}{2 n^{2}}-\frac{m \alpha^{4}}{n^{3}}\left(-\frac{1}{2 l+2}+\frac{1}{8 n}\right), \text { for } l=j-1 \geq 1 .
\end{gathered}
$$

These expressions coincide with those for the spin-mixed $l=j \geq 1$ states. The exact one body Dirac spectrum for "hydrogen" with an 
infinitely heavy point "proton" bound by only a scalar interaction, when expanded out through terms of order $\alpha^{4}$, is

$$
\epsilon_{B}=-\frac{m \alpha^{2}}{2 n^{2}}-\frac{m \alpha^{4}}{n^{3}}\left(-\frac{1}{2|k|}+\frac{1}{8 n}\right),
$$

in which $k=l+1$ for $j=l+\frac{1}{2}$ and $k=-l$ for $j=l-\frac{1}{2}$. In the case of scalar interaction, in the limit $m_{2} \rightarrow \infty$, the two-body constraint results (5.52)-(5.60) produce exactly this one-body result.

\section{Summary}

In this section we have used a perturbative treatment of the Schrödinger form of the twi)-body Dirac equations to derive the energy spectra through order $\alpha^{4}$ for relativistic two-body fermion-antifermion bound states in which the two masses are not necessarily the same, for electromagnetic, scalar, or time-like interactions arising from the corresponding Born amplitudes. Historically, the electromagnetic result was first derived with the use of the Breit equation. We have seen in this section that our perturbative treatment of the weak potential form of our Eq.(4.21a) yields results for $Q E D$ at this order that agree with the standard results given in Ref.(31). In the next section we show that a nonperturbative treatment of the general (unapproximated) equations leads to the same perturbative spectral results within an error on the order of $\mu 10^{-4,-5} \alpha^{4}$ or, roughly, of order $\mu \alpha^{6}$. Thus, the results of section $\mathrm{V}$ in combination with those of VI will show the agreement of our nonperturbative treatment of Eq.(4.21a-b) with the standard field theoretic results through this order. 
VI Nonperturbative Numerical Solution of the Two-Body Dirac Equations

We obtain the radial forms of the coupled constraint equations (4.21a-b) needed for our numerical solution for the general fermionantifermion system by using the matrix elements of the spin-dependent operators given in Appendix C. We take the general wave function to be of the form

$$
\psi_{i j m}=\sum_{l, s} c_{i l s} R_{i l s j} \mathcal{Y}_{l s j m} ; i=1,2,3,4
$$

in which $R_{i l s j}=\frac{u_{i l s j}}{r}$ is the associated radial wave function and $\mathcal{Y}_{l s j m}$ is the total angular momentum eigenfunction. The resultant Schrödingerlike equation (4.21a) for the singlet states $(j=l, s=0) u_{1 j 0 j}$ which couples this upper-upper component to $u_{1 j 1 j}$ and $u_{4 j 0 j}$ is given by

$$
\begin{aligned}
& \left\{-\frac{d^{2}}{d r^{2}}+\frac{j(j+1)}{r^{2}}+2 m_{w} S+S^{2}+2 \epsilon_{w} \mathcal{A}-\mathcal{A}^{2}+2 \epsilon_{w} \mathcal{V}-\mathcal{V}^{2}\right. \\
& \left.\left.+\ln ^{\prime} \chi_{1} \chi_{2} \frac{d}{d r}-\frac{3}{2} \partial^{2} \mathcal{G}+\frac{9}{4}\left(\mathcal{G}^{\prime}\right)^{2}+\frac{3}{2} \ln ^{\prime} \chi_{1} \chi_{2} \mathcal{G}^{\prime}\right)+\frac{1}{4}\left(J^{\prime}-L^{\prime}\right)^{2}-\frac{\ln ^{\prime} \chi_{1} \chi_{2}}{r}\right\} u_{1 j 0 j} \\
& -\frac{\ln ^{\prime}\left(\chi_{1} / \chi_{2}\right)}{r} \sqrt{\xi(1+1)} u_{1 j 1 j} \\
& +\left\{\left[-\frac{1}{2} l n^{\prime} \chi_{1} \chi_{2}(J-L)^{\prime}-\frac{3}{2} \mathcal{G}^{\prime}(J-L)^{\prime}+\frac{1}{2} \partial^{2}(J-L)\right]\right\} u_{4 j 0 j}=b^{2}(w) u_{1 j 0 j} \text {. }
\end{aligned}
$$

The corresponding equation (4.21b) for the lower-lower component $u_{4 j 0 j}$ which couples it to $u_{4 j 1 j}$ and $u_{1 j 0 j}$ takes the form

$$
\begin{gathered}
\left\{-\frac{d^{2}}{d r^{2}}+\frac{j(j+1)}{r^{2}}+2 m_{w} S+S^{2}+2 \epsilon_{w} \mathcal{A}-\mathcal{A}^{2}+2 \epsilon_{w} \mathcal{V}-\mathcal{V}^{2}\right. \\
\left.\left.+\ln ^{\prime} \bar{\chi}_{1} \bar{\chi}_{2} \frac{d}{d r}-\frac{3}{2} \partial^{2} \mathcal{G}+\frac{9}{4}\left(\mathcal{G}^{\prime}\right)^{2}+\frac{3}{2} \ln ^{\prime} \bar{\chi}_{1} \bar{\chi}_{2} \mathcal{G}^{\prime}\right)+\frac{1}{4}\left(J^{\prime}-L^{\prime}\right)^{2}-\frac{\ln ^{\prime} \bar{\chi}_{1} \bar{\chi}_{2}}{r}\right\} u_{4 j 0 j}
\end{gathered}
$$




$$
\begin{gathered}
-\frac{\ln \left(\bar{\chi}_{1} / \bar{\chi}_{2}\right)}{r} \sqrt{(\mathcal{G}(\mathcal{Y}+1)} u_{4 j 1 j} \\
+\left\{\left[-\frac{1}{2} \ln ^{\prime} \bar{\chi}_{1} \bar{\chi}_{2}(J-L)^{\prime}-\frac{3}{2} \mathcal{G}^{\prime}(J-L)^{\prime}+\frac{1}{2} \partial^{2}(J-L)\right]\right\} u_{1 j 0 j}=b^{2}(w) u_{4 j 0 j} .
\end{gathered}
$$

For $S$ states or equal mass systems, these equations decouple from those for $u_{1 j 1 j}$ and $u_{4 j 1 j}$ since $\sqrt{f(j+1)} \ln \left(\chi_{1} / \chi_{2}\right)=\sqrt{1(j+1)} \ln \left(\bar{\chi}_{1} / \bar{\chi}_{2}\right)=0$. However, for the general unequal mass case, these equations are coupled to those for the $j=l, s=1$ components $u_{1 j 1 j}$ and $u_{4 j 1 j}$. For those triplet states the coupled Schrödinger-like equations are

$$
\begin{gathered}
\left\{-\frac{d^{2}}{d r^{2}}+\frac{j(j+1)}{r^{2}}+2 m_{w} S+S^{2}+2 \epsilon_{w} \mathcal{A}-\mathcal{A}^{2}+2 \epsilon_{w} \mathcal{V}-\mathcal{V}^{2}\right. \\
\left.+\ln ^{\prime} \chi_{1} \chi_{2} \frac{d}{d r}-\frac{1}{2} \partial^{2} \mathcal{G}+\frac{1}{4}\left(\mathcal{G}^{\prime}\right)^{2}+\frac{1}{2} \ln ^{\prime} \chi_{1} \chi_{2} \mathcal{G}^{\prime}\right)+\frac{1}{4}\left(J^{\prime}-L^{\prime}\right)^{2}-\frac{\ln ^{\prime} \chi_{1} \chi_{2}}{r} \\
\left.+\frac{\ln ^{\prime} \chi_{1} \chi_{2}+\mathcal{G}^{\prime}}{r}\right\} u_{1 j 1 j}-\frac{\ln ^{\prime}\left(\chi_{1} / \chi_{2}\right)}{r} \sqrt{(\mathcal{H}+1)} u_{1 j 0 j} \\
+\left\{\left[+\frac{1}{2} \ln ^{\prime} \chi_{1} \chi_{2}(J-L)^{\prime}+\frac{1}{2} \mathcal{G}^{\prime}(J-L)^{\prime}-\frac{1}{2} \partial^{2}(J-L)\right]\right. \\
\left.+\frac{(J-L)^{\prime}}{r}\right\}{ }_{i_{4 j} 1 j}=b^{2}(w) u_{1 j 1 j}
\end{gathered}
$$

and

$$
\begin{gathered}
\left\{-\frac{d^{2}}{d r^{2}}+\frac{j(j+1)}{r^{2}}+2 m_{w} S+S^{2}+2 \epsilon_{w} \mathcal{A}-\mathcal{A}^{2}+2 \epsilon_{w} \mathcal{V}-\mathcal{V}^{2}\right. \\
\left.+\ln ^{\prime} \bar{\chi}_{1} \bar{\chi}_{2} \frac{d}{d r}-\frac{1}{2} \partial^{2} \mathcal{G}+\frac{1}{4}\left(\mathcal{G}^{\prime}\right)^{2}+\frac{1}{2} \ln ^{\prime} \bar{\chi}_{1} \bar{\chi}_{2} \mathcal{G}^{\prime}\right)+\frac{1}{4}\left(J^{\prime}-L^{\prime}\right)^{2}-\frac{\ln ^{\prime} \bar{\chi}_{1} \bar{\chi}_{2}}{r} \\
\left.+\frac{\ln ^{\prime} \bar{\chi}_{1} \bar{\chi}_{2}+\mathcal{G}^{\prime}}{r}\right\} u_{4 j 1 j}-\frac{\ln ^{\prime}\left(\bar{\chi}_{1} / \bar{\chi}_{2}\right)}{r} \sqrt{(\mathcal{J}(f+1)} u_{4 j 0 j} \\
+\left\{\left[+\frac{1}{2} \ln ^{\prime} \bar{\chi}_{1} \bar{\chi}_{2}(J-L)^{\prime}+\frac{1}{2} \mathcal{G}^{\prime}(J-L)^{\prime}-\frac{1}{2} \partial^{2}(J-L)\right]\right.
\end{gathered}
$$




$$
\left.+\frac{(J-L)^{\prime}}{r}\right\} u_{1 j 1 j}=b^{2}(w) u_{4 j 1 j}
$$

Next we write out the four coupled equations for the two triplet states $j=l \pm 1$. Eq.(4.14a) for the triplet states $(s=1, l=j-1) u_{1 j-11 j}$, which couples this upper-upper component wave function to $u_{1 j+11 j}, u_{4 j+11 j}$, and $u_{4 j-11 j}$, becomes

$$
\begin{aligned}
& \left\{\cdots \frac{d^{2}}{d r^{2}}+\frac{j(j-1)}{r^{2}}+2 m_{w} S+S^{2}+2 \epsilon_{w} \mathcal{A}-\mathcal{A}^{2}+2 \epsilon_{w} \mathcal{V}-\mathcal{V}^{2}\right. \\
& +\ln ^{\prime} \chi_{1} \chi_{2} \frac{d}{d r}-\frac{\partial^{2} \mathcal{G}}{2(2 j+1)}+\frac{1}{4}\left(\mathcal{G}^{\prime}\right)^{2}+\frac{\mathcal{G}^{\prime} l n^{\prime} \chi_{1} \chi_{2}}{2(2 j+1)}+\frac{1}{4}\left(J^{\prime}-L^{\prime}\right)^{2}-\frac{\ln ^{\prime} \chi_{1} \chi_{2}}{r} \\
& \left.-(j-1) \frac{\ln ^{\prime} \chi_{1} \chi_{2}+\mathcal{G}^{\prime} /(2 j+1)}{r}\right\} u_{1 j-11 j} \\
& +\frac{\sqrt{j(j+1)}}{2 j+1}\left[-\left(\mathcal{G}^{\prime \prime}-\frac{\mathcal{G}^{\prime}}{r}\right)+\ln ^{\prime} \chi_{1} \chi_{2} \mathcal{G}^{\prime}\right] u_{1 j+11 j} \\
& +\left\{\left(\frac{\ln ^{\prime} \chi_{1} \chi_{2}}{(2 j+1)}+\mathcal{G}^{\prime}\right) \frac{(J-L)^{\prime}}{2}-\frac{\partial^{2}(J-L)}{2(2 j+1)}-\frac{(j-1)}{(2 j+1)} \frac{(J-L)^{\prime}}{r}\right\} u_{4 j-11 j} \\
& \left.+\frac{\sqrt{j(j+1)}}{2 j+1}\left[(J-L)^{\prime}\left(\ln ^{\prime} \chi_{1} \chi_{2}+\frac{1}{r}\right)-(J-L)^{\prime \prime}\right]\right\} u_{4 j+11 j}=b^{2}(w) u_{1 j-11 j}
\end{aligned}
$$

The corresponding equation (4.14b) for the lower-lower component becomes

$$
\begin{gathered}
\left\{-\frac{d^{2}}{d r^{2}}+\frac{j(j-1)}{r^{2}}+2 m_{w} S+S^{2}+2 \epsilon_{w} \mathcal{A}-\mathcal{A}^{2}+2 \epsilon_{w} \mathcal{V}-\mathcal{V}^{2}\right. \\
+\ln ^{\prime} \bar{\chi}_{1} \bar{\chi}_{2} \frac{d}{d r}-\frac{\partial^{2} \mathcal{G}}{2(2 j+1)}+\frac{1}{4}\left(\mathcal{G}^{\prime}\right)^{2}+\frac{\mathcal{G}^{\prime} \ln ^{\prime} \bar{\chi}_{1} \bar{\chi}_{2}}{2(2 j+1)}+\frac{1}{4}\left(J^{\prime}-L^{\prime}\right)^{2}-\frac{\ln ^{\prime} \bar{\chi}_{1} \bar{\chi}_{2}}{r} \\
\left.-(j-1) \frac{\ln ^{\prime} \bar{\chi}_{1} \bar{\chi}_{2}+\mathcal{G}^{\prime} /(2 j+1)}{r}\right\} u_{4 j-11 j}
\end{gathered}
$$




$$
\begin{gathered}
+\frac{\sqrt{j(j+1)}}{2 j+1}\left[-\left(\mathcal{G}^{\prime \prime}-\frac{\mathcal{G}^{\prime}}{r}\right)+\ln ^{\prime} \bar{\chi}_{1} \bar{\chi}_{2} \mathcal{G}^{\prime}\right] u_{4 j+11 j} \\
+\left\{\left(\frac{\ln ^{\prime} \bar{\chi}_{1} \bar{\chi}_{2}}{(2 j+1)}+\mathcal{G}^{\prime}\right) \frac{(J-L)^{\prime}}{2}-\frac{\partial^{2}(J-L)}{2(2 j+1)}-\frac{(j-1)}{(2 j+1)} \frac{(J-L)^{\prime}}{r}\right\} u_{1 j-11 j} \\
\left.+\frac{\sqrt{j(j+1)}}{2 j+1}\left[(J-L)^{\prime}\left(\ln ^{\prime} \bar{\chi}_{1} \bar{\chi}_{2}+\frac{1}{r}\right)-(J-L)^{\prime \prime}\right]\right\} u_{1 j+11 j}=b^{2}(w) u_{4 j-11 j} .
\end{gathered}
$$

Eqs.(6.3a-b) are coupled to the corresponding two equations for the triplet $s=1, l=j+1$ states given by

$$
\begin{gathered}
\left\{-\frac{d^{2}}{d r^{2}}+\frac{(j+1)(j+2)}{r^{2}}+2 m_{w} S+S^{2}+2 \epsilon_{w} \mathcal{A}-\mathcal{A}^{2}+2 \epsilon_{w} \mathcal{V}-\mathcal{V}^{2}\right. \\
+\ln ^{\prime} \chi_{1} \chi_{2} \frac{d}{d r}+\frac{\partial^{2} \mathcal{G}}{2(2 j+1)}+\frac{1}{4}\left(\mathcal{G}^{\prime}\right)^{2}-\frac{\mathcal{G}^{\prime} l n^{\prime} \chi_{1} \chi_{2}}{2(2 j+1)}+\frac{1}{4}\left(J^{\prime}-L^{\prime}\right)^{2}-\frac{\ln ^{\prime} \chi_{1} \chi_{2}}{r} \\
\left.+(j+2) \frac{l^{\prime} \chi_{1} \chi_{2}-\mathcal{G}^{\prime} /(2 j+1)}{r}\right\} u_{1 j+11 j} \\
+\frac{\sqrt{j(j+1)}}{2 j+1}\left[-\left(\mathcal{G}^{\prime \prime}-\frac{\mathcal{G}^{\prime}}{r}\right)+\ln ^{\prime} \chi_{1} \chi_{2} \mathcal{G}^{\prime}\right] u_{1 j-11 j} \\
+\left\{\left(-\frac{\ln { }^{\prime} \chi_{1} \chi_{2}}{(2 j+1)}+\mathcal{G}^{\prime}\right) \frac{(J-L)^{\prime}}{2}+\frac{\partial^{2}(J-L)}{2(2 j+1)}-\frac{(j+2)}{(2 j+1)} \frac{(J-L)^{\prime}}{r}\right\} u_{4 j+11 j} \\
\left.+\frac{\sqrt{j(j+1)}}{2 j+1}\left[(J-L)^{\prime}\left(\ln ^{\prime} \chi_{1} \chi_{2}+\frac{1}{r}\right)-(J-L)^{\prime \prime}\right]\right\} u_{4 j-11 j}=b^{2}(w) u_{1 j+11 j}
\end{gathered}
$$

and

$$
\begin{gathered}
\left\{-\frac{d^{2}}{d r^{2}}+\frac{(j+1)(j+2)}{r^{2}}+2 m_{w} S+S^{2}+2 \epsilon_{w} \mathcal{A}-\mathcal{A}^{2}+2 \epsilon_{w} \mathcal{V}-\mathcal{V}^{2}\right. \\
+\ln ^{\prime} \bar{\chi}_{1} \bar{\chi}_{2} \frac{d}{d r}+\frac{\partial^{2} \mathcal{G}}{2(2 j+1)}+\frac{1}{4}\left(\mathcal{G}^{\prime}\right)^{2}-\frac{\mathcal{G}^{\prime} l^{\prime} \bar{\chi}_{1} \bar{\chi}_{2}}{2(2 j+1)}+\frac{1}{4}\left(J^{\prime}-L^{\prime}\right)^{2}-\frac{\ln ^{\prime} \bar{\chi}_{1} \bar{\chi}_{2}}{r}
\end{gathered}
$$




$$
\begin{gathered}
\left.+(j+2) \frac{\ln ^{\prime} \bar{\chi}_{1} \bar{\chi}_{2}-\mathcal{G}^{\prime} /(2 j+1)}{r}\right\} u_{4 j+11 j} \\
+\frac{\sqrt{j(j+1)}}{2 j+1}\left[-\left(\mathcal{G}^{\prime \prime}-\frac{\mathcal{G}^{\prime}}{r}\right)+\ln ^{\prime} \bar{\chi}_{1} \bar{\chi}_{2} \mathcal{G}^{\prime}\right] u_{4 j-11 j} \\
+\left\{\left(-\frac{\ln ^{\prime} \bar{\chi}_{1} \bar{\chi}_{2}}{(2 j+1)}+\mathcal{G}^{\prime}\right) \frac{(J-L)^{\prime}}{2}+\frac{\partial^{2}(J-L)}{2(2 j+1)}-\frac{(j+2)}{(2 j+1)} \frac{(J-L)^{\prime}}{r}\right\} u_{1 j+11 j} \\
\left.+\frac{\sqrt{j(j+1)}}{2 j+1}\left[(J-L)^{\prime}\left(\ln ^{\prime} \bar{\chi}_{1} \bar{\chi}_{2}+\frac{1}{r}\right)-(J-L)^{\prime \prime}\right]\right\} u_{1 j-11 j}=b^{2}(w) u_{4 j+11 j} .
\end{gathered}
$$

For the ${ }^{3} P_{0}$ states there are only two coupled equations:

$$
\begin{gathered}
\left\{-\frac{d^{2}}{d r^{2}}-\frac{2}{r} \frac{d}{d r}+\frac{2}{r^{2}}+2 m_{w} S+S^{2}+2 \epsilon_{w} \mathcal{A}-\mathcal{A}^{2}+2 \epsilon_{w} \mathcal{V}-\mathcal{V}^{2}\right. \\
+\ln ^{\prime} \chi_{1} \chi_{2} \frac{d}{d r}+\frac{1}{2} \partial^{2} \mathcal{G}+ \\
\frac{1}{4}\left(\mathcal{G}^{\prime}\right)^{2}-\frac{1}{2} \ln ^{\prime} \chi_{1} \chi_{2} \mathcal{G}^{\prime}+\frac{1}{4}\left(J^{\prime}-L^{\prime}\right)^{2}-\frac{\ln ^{\prime} \chi_{1} \chi_{2}}{r} \\
\left.+2 \frac{\ln ^{\prime} \chi_{1} \chi_{2}-\mathcal{G}^{\prime}}{r}\right\} u_{1101} \\
-\left\{\frac{1}{2} \ln ^{\prime} \chi_{1} \chi_{2}(J-L)^{\prime}+\frac{1}{2} \mathcal{G}^{\prime}(J-L)^{\prime}+\frac{1}{2} \partial^{2}(J-L)-2 \frac{(J-L)^{\prime}}{r}\right\} u_{4101} \\
=b^{2}(w) u_{1101}
\end{gathered}
$$

and

$$
\begin{gathered}
\left\{-\frac{d^{2}}{d r^{2}}-\frac{2}{r} \frac{d}{d r}+\frac{2}{r^{2}}+2 m_{w} S+S^{2}+2 \epsilon_{w} \mathcal{A}-\mathcal{A}^{2}+2 \epsilon_{w} \mathcal{V}-\mathcal{V}^{2}\right. \\
+\ln ^{\prime} \bar{\chi}_{1} \bar{\chi}_{2} \frac{d}{d r}+\frac{1}{2} \partial^{2} \mathcal{G}+\frac{1}{4}\left(\mathcal{G}^{\prime}\right)^{2}-\frac{1}{2} l^{\prime} \bar{\chi}_{1} \bar{\chi}_{2} \mathcal{G}^{\prime}+\frac{1}{4}\left(J^{\prime}-L^{\prime}\right)^{2}-\frac{\ln ^{\prime} \bar{\chi}_{1} \bar{\chi}_{2}}{r} \\
\left.+2 \frac{\ln ^{\prime} \bar{\chi}_{1} \bar{\chi}_{2}-\mathcal{G}^{\prime}}{r}\right\} u_{4101} \\
-\left\{\frac{1}{2} \ln ^{\prime} \bar{\chi}_{1} \bar{\chi}_{2}(J-L)^{\prime}+\frac{1}{2} \mathcal{G}^{\prime}(J-L)^{\prime}+\frac{1}{2} \partial^{2}(J-L)-2 \frac{(J-L)^{\prime}}{r}\right\} u_{1101} \\
=b^{2}(w) u_{4101} .
\end{gathered}
$$


Note that in each of the equations (6.2-6.4), the quasipotential couples the upper-upper component to the lower-lower component.

These sets of coupled equations, for the ${ }^{3} P_{0}$ case and the equal mass (or $l=0$ ) $j=l$ case, all take the general form of two coupled Schrödinger-like equations:

$$
\begin{gathered}
{\left[-\frac{d^{2}}{d r^{2}}+f(r) \frac{d}{d r}+q(r)\right] u(r)+g(r) v(r)=b^{2} u(r),} \\
{\left[-\frac{d^{2}}{d r^{2}}+e(r) \frac{d}{d r}+s(r)\right] v(r)+a(r) u(r)=b^{2} v(r),}
\end{gathered}
$$

Likewise the four coupled equations for all other cases take the general form

$$
\begin{aligned}
& {\left[-\frac{d^{2}}{d r^{2}}+f(r) \frac{d}{d r}+q(r)\right] u(r)+g(r) v(r)+c(r) y(r)+p(r) z(r)=b^{2} u(r),} \\
& {\left[-\frac{d^{2}}{d r^{2}}+f(r) \frac{d}{d r}+s(r)\right] v(r)+a(r) u(r)+h(r) y(r)+\beta(r) z(r)=b^{2} v(r),} \\
& {\left[-\frac{d^{2}}{d r^{2}}+e(r) \frac{d}{d r}+\sigma(r)\right] y(r)+k(r) u(r)+l(r) v(r)+j(r) z(r)=b^{2} y(r),} \\
& \left.\left[-\frac{d^{2}}{d r^{2}}+e(r) \frac{d}{d r}+t(r)\right] z(r)+m(r) u(r)+n(r) v(r)+o(r) y(r)=b^{2} z(r) .6 c\right)
\end{aligned}
$$

In the final version of the equations that we will use in the numerical work we transform the independent variable to $x=\ln \left(r / r_{0}\right)$ in which $r_{0}$ is a numerical constant times the Compton wave length. As a result our general set of equations takes the form

$$
\left[-\frac{1}{r^{2}} \frac{d^{2}}{d x^{2}}+\left(\frac{2}{r^{2}}+\frac{f}{r}\right) \frac{d}{d x}-\frac{f}{2 r}+q-\frac{3}{4 r^{2}}\right] u(x)+g v(x)=b^{2} u(x),
$$




$$
\left[-\frac{1}{r^{2}} \frac{d^{2}}{d x^{2}}+\left(\frac{2}{r^{2}}+\frac{e}{r}\right) \frac{d}{d x}-\frac{e}{2 r}+s-\frac{3}{4 r^{2}}\right] v(r)+a u(x)=b^{2} v(x) .
$$

for the case of two coupled equations, and

$$
\begin{aligned}
& {\left[-\frac{1}{r^{2}} \frac{d^{2}}{d x^{2}}+\left(\frac{2}{r^{2}}+\frac{f}{r}\right) \frac{d}{d x}-\frac{f}{2 r}+q-\frac{3}{4 r^{2}}\right] u(r)+g v(x)+c y(x)+p z(x)=b^{2} u(x),} \\
& {\left[-\frac{1}{r^{2}} \frac{d^{2}}{d x^{2}}+\left(\frac{2}{r^{2}}+\frac{f}{r}\right) \frac{d}{d x}-\frac{f}{2 r}+s-\frac{3}{4 r^{2}}\right] v(r)+a u(x)+h y(x)+\beta z(x)=b^{2} v(x),} \\
& {\left[-\frac{1}{r^{2}} \frac{d^{2}}{d x^{2}}+\left(\frac{2}{r^{2}}+\frac{e}{r}\right) \frac{d}{d x}-\frac{e}{2 r}+\sigma-\frac{3}{4 r^{2}}\right] y(x)+k u(x)+l v(x)+j z(x)=b^{2} y(x),} \\
& {\left[-\frac{1}{r^{2}} \frac{d^{2}}{d x^{2}}+\left(\frac{2}{r^{2}}+\frac{e}{r}\right) \frac{d}{d x}-\frac{e}{2 r}+t-\frac{3}{4 r^{2}}\right] z(x)+m u(x)+n v(x)+o y(x)=b^{2} z(x) .}
\end{aligned}
$$

for the case of four coupled equations. To obtain this form of these equations we have also scaled the dependent variables. In order to determine the eigenvalue we use a numerical technique that employs an adaptation of the central difference approximation ${ }^{52}$ combined with the inverse power method. Although its application to uncoupled Schrödinger-like equations requires the inversion of a large banded matrix, the procedure is straightforward $d^{53}$. Extension of this technique to two and four coupled Schrödinger-like equations requires the inversion of a large blocked matrix with banded diagonal blocks and diagonal off diagonal blocks. The details of that procedure will be presented in a separate publication.

In the tables below we give the numerical results for electromagnetic interactions (QED) obtained from (6.2-4) with $\mathcal{A}=-\alpha / r$, and $S=\mathcal{V}=$ 0 so that $\mathcal{G}=-J=\ln (1+\alpha /(w r)), L=0$ where $\alpha$ is $1 / 137.0359895(61)$. We present results for numerical calculations for muonium $\left(e^{-} \mu^{+}\right)$and positronium $\left(e^{-} e^{+}\right)$. We do not, however, include the effects of the 
annihilation diagram for the $e^{-} e^{+}$system in the tests presented below. A Fierz transformation of the annihilation diagram to quasipotential form would include pseudoscalar and pseudovector couplings in addition to scalar and vector couplings which would require a generalization of the interactions contained in the two-body Dirac equations considered in this paper ${ }^{54}$.

In table I we present the binding energies in electron volts for the $n=1,2,3$ levels for the $e^{+} e^{-}$system $^{55}$. The quantum numbers are those of the upper-upper component of the system wave function $\psi_{i j m}$. In the first four columns are the values of the quinium numbers $l, s, j, n$. In the fifth column is the number of coupled equations $N_{c}$ that are included in the numerical test. When $N_{c}=1$, we use just one equation, the one for the upper-upper component, with the couplings to the lower-lower component and $l$-mixing neglected. The case $N_{c}=2$ corresponds to the fully coupled system (upper-upper and lower-lower) of equations for the singlet states, the ${ }^{3} P_{0}$ states and the triplet $j=l$ states. For the other triplet states in the $N_{c}=2$ case we neglect either the coupling due to angular momentum mixing or that due to the coupling between $\psi_{1}$ and $\psi_{4}$. To distinguish between these $N_{c}=2$ cases for the nonperturbative (numerical) test, we let $M$ stand for the neglect of the $l=j+1, j-1$ coupling while $C$ stands for the neglect of the coupling between the upper-upper and lower-lower components. The $N_{c}=4$ case corresponds to the fully coupled triplet states for $l=j+1, j-1, j \neq 0$ in which couplings between the upper-upper and lower-lower component as well as the $l$ mixing are not neglected. In the next column are the energy levels obtained from the perturbative expansions given in section $\mathrm{V}$ by 
Eqs.(5.18-25) (which involve only a single, uncoupled equation (5.9)). In the next to last column are the nonperturbative numerical results from Eqs.(6.2-4), the most important results of the paper. The last column gives the differences between the perturbative and numerical calculations divided by $\mu \alpha^{4} / n^{3}$. Since we are not including radiative corrections, these differences should be on the order of $\mu \alpha^{6}$ (as opposed to radiative corrections on the order of $\mu \alpha^{5}$ or $\mu \alpha^{5} \ln \alpha$ ) when all of the couplings in Eqs.(6.2-4) are included. Thus the entries in this difference column for the full coupled equations (two or four depending on the quantum numbers) should be on the order of $\alpha^{2}$ or $10^{-4,-5}$.

Table II gives the binding energies in electron voits for $n=1,2,3$ levels for muonium $\left(e^{-} \mu^{+}\right)$. The columns are labeled as before except that $M$ stands for the neglect of $l$ mixing ( for $l=j+1, j-1$ ) or $s$ mixing $(l=j \geq 1)$ in the triplet $N_{c}=2$ cases. Note that for muonium the $N_{c}=2$ case corresponds to the fully coupled system only for the $j=0$ states (singlet or triplet). For all other states the fully coupled constraint equations correspond to $N_{c}=4$ with the combined coupling for upperupper and lower-lower components, and for $l$ mixing ( for $l=j+1, j-1$ ) or $s$ mixing $(l=j)$,

For the cases of scalar and pur sly time-like interactions, we present just the ground state results for equal masses. In Table III we give the numerical results for scalar interactions obtained from (6.2-4) with $S=-\alpha / r$, and $\mathcal{V}=\mathcal{A}=0$ so that $\mathcal{G}=-J=0$ with $L$ given by (3.48a). The results in the perturbative column are the energy levels obtained from the perturbative expansions given in section $\mathrm{V}$ by Eqs.(5.26-32) (which employ only a single, uncoupled equation (5.12)). 
In table IV we give the numerical results for time-like interactions obtained from (6.2-4) with $\mathcal{V}=-\alpha / r$, and $S=\mathcal{A}=0$ so that $\mathcal{G}=L=0$ with $J$ given by (3.48b). The results in the perturbative column are the energy levels obtained from the perturbative expansions given in section $\mathrm{V}$ by Eqs.(5.33-39) (which employ only a single, uncoupled equation $(5.15))$.

Let us examine these results more closely. Note that in all cases for given values of $l, s, j$, and $n$, the agreement between the nonperturbative numerical result for the fully coupled system of equations (highest value of $N_{c}$ ) and the perturbative result is excellent. Typically, the differences are on the order of $\mu \alpha^{6}$. As we neglect couplings in the strong potential form of the equations, Eqs.(6.2-4), this agreement is spoiled to one degree or another, except in cases when the agreement between the single uncoupled equation and the perturbative result is already excellent. Thus, when effects due to these couplings are significant, they conspire in a complex fashion to produce agreement with the perturbative result. In the case of the electromagnetic interaction for the ${ }^{1} S_{0}$ positronium states, the coupling between the upper-upper and lowerlower wave functions is crucial in order to obtain agreement through order $\alpha^{4}$ with the perturbatively computed spectral results (or for that matter with the exact solution obtained by other methods ${ }^{16}$ ). Without them, the error is on the order of $5 \%$ of $\mu \alpha^{4}$, much larger than the order $\mu \alpha^{6}$ error expected. The same phenomenon occurs for the ${ }^{3} S_{1}$ states in positronium except that in this case the (off-diagonal) tensor coupling is needed in conjunction with the coupling between the upper-upper and lower-lower components. (Note that in the perturbative treatment of the 
equation, the latter coupling to the lower-lower component played no role whatsoever). Neither coupling by itself is sufficient to produce a result accurate enough so that the errors are on the order of $\alpha^{6}$. For the equal mass $l>0$ states however, it is not necessary to include the effects of either the (off diagonal) tensor coupling or that between the upper-upper and lower-lower components to obtain good agreement. The uncoupled upper-upper equation by itself is sufficient.

For muonium, the coupling between the upper-upper and lowerlower components is crucial for the $l=0$ states just as it was in the equal mass case. Again the agreement improves by two or three orders of magnitude when the coupling to the lower-lower component is included. This improvement might at first not seem significant since the relative error starts at an already respectable $10^{-5} \sim \alpha^{2}$ so that further improvement to $\sim 10^{-7}$ may appear meaningless. However, a glance at the perturbative spectrum reveals that the smallest corrections are the recoil corrections $\sim \mu^{2} \alpha^{4} / M$. Relative to these the correction due to the coupling to the lower-lower component becomes as significant as in the equal mass case. Note also that the spin-mixing coupling between the ${ }^{1} L_{l}$ and ${ }^{3} L_{l}$ states is crucial to obtain agreement between the nonperturbative and perturbative treatments of our equations. This coupling was also important to obtain agreement between our perturbative results and standard treatments. Further, in both the equal and unequal mass cases, we have ignored the coupling to the lower-lower component in the perturbative calculations. Its nonperturbative importance for $S$ states thus comes as a surprise. We find that couplings to different components of the wave equation that are numerically important for the nonpertur- 
bative calculation are not important for the perturbative calculation ${ }^{56}$.

The perturbative treatment of our equations for the scalar and purely time-like interactions generates no hyperfine splitting through or$\operatorname{der} \alpha^{4}$. The nonperturbative numerical results are consistent with this but again only if all couplings are included. (The lack of hyperfine splitting holds as well for the nonperturbative treatment of the unequal mass cases.)

Besides the coupling, what other relativistic strong-potential structures are crucial for the excellent agreement we have obtained? We have referred earlier to strong potential terms as those in Eqs.(4.21a-b) beyond the collective minimal (Todorov) part (e.g. $+2 \epsilon_{w} \mathcal{A}-\mathcal{A}^{2}$ for electromagnetic interaction). "Relativistic strong-potential structures" refers collectively to these terms, in particular to the potential energy dependences in denominators appearing in those terms of the form $E_{i}+M_{i}, G^{2}$. In the weak-potential limit (Eqs.(5.1-5.6)), for $\mathcal{A}=-\alpha / r$, these terms become singular potentials (ones more attractive than $-1 / 4 r^{2}$ near the origin) but are themselves non-singular in the constraint equation Eq.(4.21ab). Singular potentials appear in our formalism only as a result of perturbative approximation, when the strong-potential terms such as $-\frac{\ln ^{\prime} \chi_{1}}{r}, \ln ^{\prime} \chi_{1} \frac{d}{d r},-\partial^{2} \mathcal{G}$ that appear in $(4.21 \mathrm{a}-\mathrm{b})$ are treated as weak. In such a perturbative approximation, attractive potential energy terms with a radial dependence of the form $\frac{1}{r^{3}}, \frac{1}{r^{2}} \frac{d}{d r}$ and $\delta(\vec{r})$ arise as typical relativistic weak-potential limits of the relativistic strong potential terms. Those limits can only be treated perturbatively, using well behaved unperturbed wave functions. Otherwise a nonperturbative treatment of these singular potentials would lead to nonnormalizable singular 
wave functions. However, the unapproximated strong-potential terms $-\frac{\ln ^{\prime} \chi_{1}}{r}, \ln ^{\prime} \chi_{1} \frac{d}{d r},-\partial^{2} \mathcal{G}$, etc. in our equation, from which the singular potentials originate, are well behaved for small $r$, since the logarithmic derivatives generate denominators that moderate the small distance be havior. Thus the unapproximated terms do not lead to singular wave functions. For example in the case of $Q E D$, consider terms with a radial dependence of the form

$$
-\frac{\ln ^{\prime} \chi_{1}}{r}=\frac{\mathcal{A}^{\prime}}{r} \frac{\left(1+m_{1} /\left(w G^{3}\right)\right)}{\epsilon_{1}-\mathcal{A}+m_{1} / G} .
$$

For $\mathcal{A}=-\alpha / r$ and $G^{2}$ defined in (2.10c), this becomes $1 / r^{2}$ for small $r$ instead of $\alpha /\left(2 m_{1} r^{3}\right)$ as it does in the weak-potential approximation in which the potential dependence in the denominator is left out. The $1 / r^{2}$ behavior gives acceptable nonperturbative numerical solutions when combined with the centrifugal barrier term, whether the sign of this term is positive or negative. On the other hand the $1 / r^{3}$ dependence would not give any convergent nonperturbative numerical solution when the coefficient is negative, as can happen for QED interactions in the ${ }^{3} P_{0}$ case. So the nonsingular short distance behavior ${ }^{57}$ is crucial for every term in the quasipotential that appears in Eqs.(4.21a-b) since using the weak potential approximation in any one of the terms could render the equation as a whole ill defined quantum mechanically. Those terms, which include Darwin and spin-dependent and relativistic recoil terms (ones that vanish when one of the massis $\rightarrow \infty$ ), yield important contributions to the calculated spectra.

Just what are the distance scales at which the potentials in the various denominator terms become important? For the equal mass case the 
invariant $\mathcal{A}$ becomes comparable to the electron mass and the energy terms in the denominator at distance scales of the order of the classical electron radius, well inside the Compton wavelength. Such short distance behavior of the quasipotential is important since without this radial dependence in the denominator the correct spectral results could not be reproduced in a nonperturbative treatment. For example, if one artificially replaces $\mathcal{A}$ by a constant in the non-coulombic part of the quasipotential at a distance $r$ less than a Compton wavelength, then the $S$ state nonperturbative spectral results will no longer agree with their perturbative counterparts to the required accuracy, even though the $P$ state results will. Thus the minimal interaction constraint equations provide a natural cutoff mechanism that is essential for a nonperturbative treatment of the equation.

Before proceeding to the next section on the ${ }^{3} P_{0}$ scattering states we discuss further the fact that different parts of the two-body wave equation appear to be important for the nonperturbative calculation that are not important for the perturbative calculation. Even though this is an unexpected feature, something analogous does occur in the case of the one-body Dirac equation. For vector interactions alone (generated by either $\mathcal{A}$ or $\mathcal{V}$ ), the Pauli-form of the Dirac equation for the upper component is (Eq.(4.21a) with $m_{2} \rightarrow \infty$ )

$$
\left\{p^{2}+2 \epsilon_{1} \mathcal{A}-\mathcal{A}^{2}+i \ln ^{\prime} \chi_{1} \hat{r} \cdot p-\frac{\ln ^{\prime} \chi_{1}}{r} L \cdot \sigma_{1}\right\} \psi=\left(\epsilon_{1}^{2}-m_{1}^{2}\right) \psi
$$

where $\chi_{1}=\epsilon_{1}-\mathcal{A}+m_{1}$. If we make a scale change to eliminate the $i \hat{r} \cdot p$ term and specialize to $\mathcal{V}=-Z \alpha / r$ (corresponding to hydrogen-like 
systems without hyperfine structure) Eq. (6.10) becomes

$$
\begin{aligned}
{\left[\vec{p}^{2}-2 \epsilon_{1} \frac{Z \alpha}{r}-\right.} & \frac{Z^{2} \alpha^{2}}{r^{2}}+\frac{2 \pi Z \alpha \delta^{3}(\vec{r})}{\epsilon_{1}+m_{1}+Z \alpha / r}+\frac{3}{4} \frac{Z^{2} \alpha^{2}}{r^{4}} \frac{1}{\left(\epsilon_{1}+m_{1}+Z \alpha / r\right)^{2}} \\
& \left.+\frac{\vec{L} \cdot \vec{\sigma}_{1} Z \alpha}{r^{3}\left(\epsilon_{1}+m_{1}+Z \alpha / r\right)}\right] \psi=\left(\epsilon_{1}^{2}-m_{1}^{2}\right) \psi
\end{aligned}
$$

The term involving $\delta^{3}(\vec{r})$ gives no contribution when the $Z \alpha / r$ term in the denominator is kept. This equation can be solved exactly. The resulting spectrum yields the standard fine structure effects in hydrogenlike atoms through order $\alpha^{4}$. The weak-potential approximation of this equation, obtained by setting $\epsilon_{1}+m_{1}+Z \alpha / r$ equal to $2 m_{1}$ and neglecting the term proportional to $Z^{2} \alpha^{2} / r^{4}$, is

$$
\left(\vec{p}^{2}-2 \frac{\epsilon_{1} Z \alpha}{r}-\frac{Z^{2} \alpha^{2}}{r^{2}}+\frac{\pi Z \alpha \delta^{3}(\vec{r})}{m_{1}}+\frac{\vec{L} \cdot \vec{\sigma}_{1} Z \alpha}{2 m_{1} r^{3}}\right) \psi=\left(\epsilon_{1}^{2}-m_{1}^{2}\right) \psi
$$

Eq.(6.12) also reproduces the standard fine-structure effects in hydrogenlike atoms, but only when the three terms after the Coulomb term are treated (in fact the second and third terms can only be treated) perturbatively. Note that the weak-potential delta function term is sufficient for perturbative bound state calculations but is not adequate for nonperturbative (e.g. numerical) calculations. In particular, note that a comparison of (6.12) with (6.11) shows that, for $S$ states, where the spin-orbit term vanishes, the perturbative effect of the delta function term in (6.12) is reproduced nonperturbatively by the $3 Z^{2} \alpha^{2} /\left(4 r^{4}\left(\epsilon_{1}+m_{1}+Z \alpha / r\right)^{2}\right)$ term in (6.11). This term cannot be approximated by $\frac{3 Z^{2} \alpha^{2}}{16 m_{1}^{2} r^{4}}$ and then treated as a perturbation because the perturbative contribution of $r^{-4}$ is undefined for S-states. It must be treated nonperturbatively. So even for the ordinary one-body Dirac equation we have an instance for which 
terms that do not contribute to the spectrum in a perturbative treatment are crucial for producing the same results when the equation is treated nonperturbatively ${ }^{58}$. 


\section{Theoretical Predictions for the ${ }^{3} P_{0}$ Scattering States}

A few years ago, several groups ${ }^{19-24}$ observed anomalous positron peaks at positron kinetic energies of $250-400 \mathrm{keV}$ in heavy-ion collisions with a united-atom charge $Z>163$ for collision energies near the Coulomb barrier. The energies of the peaks seem to be nearly independent of the projectile and target combinations. Electrons were found in coincidence with the positrons with about the same energy as the positrons at the anomalous peak ${ }^{19}$. These observations were interpreted as resulting from the formation of a neutral particle or composite with subsequent decay into a positron and an electron ${ }^{21,59-61}$. Such an intermediate state could either be the product of new (non-electromagnetic) forces or hidden features of old (electromagnetic) forces. The authors of Refs. (59-61) attributed the anomaly to the nonelectromagnetic production and decay of a pseudoscalar axion. Other authors ${ }^{62-63}$ proposed the participation of new phases of QED with a larger coupling constant. Not wishing to invoke new forces, Wong and Becker ${ }^{18}$ speculated that short distance, strong potential, relativistic effects in QED might generate a resonant composite state of the $e^{+} e^{-}$system and investigated the possible origin of such resonances using an assumed electromagnetic mechanism.

Recently, measurements of Bhabha scattering ${ }^{26}$ have failed to show the presence of such resonances with lifetimes in the range from $10^{-13}$ $-10^{-9}$ sec. That is, the Bhabha scattering results so far are consistent with the results of perturbative QED. To reconcile the electromagnetic part of these results theoretically what is needed, then, is a relativistic calculation of the phase shift at c.m. energies in the neighborhood of 
peaks seen in the heavy ion collisions. Recently, Spence and $\operatorname{Vary}^{28}$ have carried out just such a calculation using several truncated versions of the Bethe-Salpeter equation. They find a family of resonances "of zero width" in the region between 1.4 and $2.2 \mathrm{MeV}$. On the other hand we shall show that our relativistic two-body Dirac equations, despite all of their short distance, strong-potential, relativistic structures, predict no resonances in the ${ }^{3} P_{0}$ state in the relevant energy range - no deviation from ordinary Bhabha scattering.

We must now decide on the form of the equations that will best display the origins of the physics in the relativistic quasipotential. In principle we could use Eqs.(6.4a-b) (as they stand) for the ${ }^{3} P_{0}$ state in order to compute the phase shift. However, further manipulation of these equations using the first order form of the two-body Dirac equations (see Appendix D) leads to an equation for the $j=l$ states, as well as the ${ }^{3} P_{0}$ states in which the upper-upper components are completely decoupled from the lower-lower components ${ }^{64}$. This allows us to see graphically whether the effective potential develops a pocket or other structure that could produce a resonance. The equation for the ${ }^{3} P_{0}$ upper-upper component wave function found in appendix $D$ is

$$
\begin{gathered}
\left\{-\frac{d^{2}}{d r^{2}}+\frac{2}{r^{2}}-\right. \\
\frac{2 \epsilon_{w} \alpha}{r}-\left(\frac{\alpha}{r}\right)^{2}-\frac{\alpha}{r^{3} w} \frac{8}{1+2 \alpha /(r w)}+\left(\frac{\alpha}{r^{2} w}\right)^{2} \frac{8}{(1+2 \alpha / r w)^{2}} \\
\left.+16 \pi \frac{\delta^{3}(\vec{r})}{w} \frac{1}{1+2 \alpha /(r w)}\right\} u_{110}=b^{2}(w) u_{110}
\end{gathered}
$$

(Note that the delta function term will not contribute because its coefficient vanishes at $r=0$.) This is the equation from which we will obtain our numerical results. We remind the reader that this equation is dictated by the combination of two-body relativistic quantum mechanics of 
the constraint formalism with a field theoretic $\Delta$ of the Bethe-Salpeter equation. This equation has the following special features. First, this covariant Schrödinger-like form displays exact relativistic kinematics. Second, the local potential structure of Eq.(7.1) generated by $\mathcal{A}=-\alpha / r$ is determined by perturbative QED in concert with the minimal interaction form that follows from quantum mechanical gauge invariance. Third, we have shown that the short distance strong-potential structure of these equations (albeit in an equivalent form) was crucial for the accurate numerical determination of the bound state spectrum demonstrating the validity of the equations down to distances of the order of $\alpha / \mathrm{m}$. Fourth, because the kinetic and Darwin terms in Eq.(7.1) are local (unlike the three-dimensional Salpeter equation or its $O\left(1 / c^{2}\right)$ Fermi-Breit reduction), our approach provides a graphical as well as covariant way of examining the short distance behavior directly. Fifth, the effective potential (including the centrifugal potential barrier) in $\mathrm{Eq}(7.1)$ is attractive and nonsingular near the origin, having the limiting behavior $-\alpha / r^{2}$ as $r \rightarrow 0$, in contrast to the more singular terms appearing in the standard $O\left(1 / c^{2}\right)$ Fermi-Breit reduction of the Salpeter equation.

The corresponding decoupled equation (see appendix D) for the upper-upper component for the ${ }^{1} J_{j}$ states of the $e^{+} e^{-}$system is

$$
\left\{-\frac{d^{2}}{d r^{2}}+\frac{j(j+1)}{r^{2}}-\frac{2 \epsilon_{w} \alpha}{r}-\left(\frac{\alpha}{r}\right)^{2}\right\} u_{j 0 j}=b^{2}(w) u_{j 0 j}
$$

In both Eqs.(7.1) and (7.2), the effective potential is non-singular near the origin.

In order to determine whether or not there are any purely electromagnetic resonances or other nonperturbative effects in the ${ }^{3} P_{0}$ states as 
described by our equation (7.1) for the $e^{+} e^{-}$system, we need to compute the phase shifts as a function of energy and compare them with the perturbatively computed phase shifts. In sections $V$ and VI we performed a successful test of our formalism which found agreement between the ${ }^{3} P_{0}$ bound state spectral results computed perturbatively and numerically. Do we obtain agreement here between the two types of computations of the phase shifts? The general form of our equation (7.1) is

$$
\left(-\frac{d^{2}}{d r^{2}}+\frac{l(l+1)}{r^{2}}-\frac{2 \epsilon_{w} \alpha}{r}+\Delta \Phi\right) u(r)=b^{2} u(r)
$$

where $\Delta \Phi$ consists of the short range parts of the effective potential. Due to the long range nature of the effective potential in Eq.(7.3), the asymptotic form of its wave function is

$$
u(r \rightarrow \infty) \rightarrow \text { const } \times \sin (b r-\eta \ln 2 b r+\Delta)
$$

in which

$$
\Delta=\delta_{l}+\sigma_{l}-l \pi / 2
$$

while $\sigma_{l}=\arg \Gamma(l+1+i \eta)$ is the Coulomb phase shift (with $\eta=-\frac{\alpha \epsilon_{w}}{b}$ ). For the ${ }^{3} P_{0}$ state, the phase shift $\delta_{l}$ is due to $\Delta \Phi$ for the ${ }^{3} P_{0}$ state:

$$
\Delta \Phi=-\frac{\alpha^{2}}{r^{2}}-\frac{8 \alpha}{w r^{3}(1+2 \alpha / w r)}+8\left(\frac{\alpha}{r^{2} w(1+2 \alpha / w r)}\right)^{2}
$$

Before computing this nonperturbatively, we evaluate $\delta_{l}$ in perturbation theory for a few representative values of the c.m. energy $w$. In analogy to the perturbative expression for the phase shift for short range $(\alpha=0)$ potentials

$$
\delta_{l}=-b \int_{0}^{\infty} j_{l}^{2}(b r) \Delta \Phi(r) r^{2} d r
$$


when we treat $\Delta \Phi$ as a perturbation we find

$$
\delta_{l}=-b \int_{0}^{\infty} \frac{F_{l}^{2}(\eta, b r)}{b^{2} r^{2}} \Delta \Phi(r) d r
$$

when $\alpha \neq 0$. In this distorted Born approximation, $F_{l}(\eta, \rho)$ is the radial Coulomb wave function. For perturbative purposes we use the unperturbed solution which can be expanded as

$$
F_{l}(\eta, \rho)=(2 l+1) ! ! \rho C_{l}(\eta) \sum_{k=l}^{\infty} b_{k} j_{k}(\rho)
$$

where

$$
\begin{gathered}
b_{l}=1, b_{l+1}=\frac{2 l+3}{l+1} \eta \\
b_{k}=\frac{2 k+1}{k(k+1)-l(l+1)}\left\{2 \eta b_{k-1}-\frac{(k-1)(k-2)-l(l+1)}{2 k-3} b_{k-2}\right\}
\end{gathered}
$$

$j_{k}(\rho)$ is a spherical Bessel function, and

$$
C_{l}(\eta)=\frac{2^{l} \sqrt{\Gamma(l+1+\eta) e^{-\pi \eta \Gamma(l+1-i \eta)}}}{(2 l+1) !} .
$$

In our case, we approximate $\Delta \Phi$ as the weak potential form $\Delta \Phi_{0}$ given by

$$
\Delta \Phi_{0} \equiv-\frac{\alpha^{2}}{r^{2}}-\frac{8 \alpha}{w r^{3}}
$$

Substitution into Eq.(7.7b) followed by the indicated integration yields

$$
\begin{gathered}
\delta_{l}=\alpha^{2}[(2 l+1) ! !]^{2}\left[C_{l}(\eta)\right]^{2} \sum_{n=2 l}^{\infty} \sum_{k=l}^{n-l} \frac{b_{k} b_{n-k}}{n+1} \frac{\sin (2 k-n+\epsilon) \frac{\pi}{2}}{2 k-n+\epsilon} \\
+\frac{\delta \alpha b}{w}[(2 l+1) ! !]^{2}\left[C_{l}(\eta)\right]^{2} \sum_{n=2 l}^{\infty} \sum_{k=l}^{n-l} \frac{b_{k} b_{n-k}}{n(n+2)} \frac{\sin (2 k-n+1+\epsilon) \frac{\pi}{2}}{2 k-n+1+\epsilon} \frac{2}{n-2 k+1+\epsilon} .
\end{gathered}
$$


in which $\epsilon$ is a positive infinitesimal.

For our nonperturbative calculations ( with unapproximated $\Delta \Phi$ ), we use the variable phase method ${ }^{65}$ generalized here to include long range interactions. Consider the two differential equations

$$
u^{\prime \prime}+\left(b^{2}-W-\bar{W}\right) u=0
$$

and

$$
\bar{u}_{i}^{\prime \prime}+\left(b^{2}-\bar{W}\right) \bar{u}_{i}=0, i=1,2
$$

in which $u(0)=\bar{u}_{1}(0)=0$. Choose

$$
\begin{gathered}
\bar{W}(r)=-2 \epsilon_{\boldsymbol{w}} / r \\
W(r)=l(l+1) / r^{2}+\Delta \Phi
\end{gathered}
$$

so that

$$
\begin{aligned}
& \bar{u}_{1}(r \rightarrow \infty) \rightarrow \text { const } \times \sin (b r-\eta \ln 2 b r+\bar{\Delta}) \\
& \bar{u}_{2}(r \rightarrow \infty) \rightarrow \text { const } \times \cos (b r-\eta \ln 2 b r+\bar{\Delta})
\end{aligned}
$$

in which

$$
\bar{\Delta}=\sigma_{0}
$$

and

$$
\begin{gathered}
u(r \rightarrow \infty) \rightarrow \text { const } \times \sin (b r-\eta \ln 2 b r+\Delta) \\
\Delta=\delta_{l}+\sigma_{l}-l \pi / 2 .
\end{gathered}
$$

In the variable phase method, one obtains a nonlinear first order differential equation for the phase shift function $\delta_{l}(r)$ such that

$$
\delta_{l}(\infty)=\delta_{l}
$$


and $\delta_{l}(0)=0$. This is done by rewriting $u(r)$ as

$$
u(r)=\alpha(r)\left[\cos \gamma(r) \bar{u}_{1}(r)+\sin \gamma(r) \bar{u}_{2}(r)\right]
$$

so that

$$
\Delta=\bar{\Delta}+\gamma(\infty)
$$

Since we have written $u(r)$ in terms of two arbitrary functions we are free to impose a condition relating them:

$$
u^{\prime}(r)=\alpha(r)^{\prime}\left[\cos \gamma(r) \bar{u}_{1}^{\prime}(r)+\sin \gamma(r) \bar{u}_{2}^{\prime}(r)\right]
$$

Combination of these two equations leads to

$$
\gamma(r)=-\tan ^{-1}\left[\frac{u(r) \bar{u}_{1}^{\prime}(r)-u^{\prime}(r) \bar{u}_{1}(r)}{u(r) \bar{u}_{2}^{\prime}(r)-u^{\prime}(r) \bar{u}_{2}(r)}\right]
$$

in which $\gamma(0)=0$. In our case

$$
\begin{gathered}
u_{1}(r)=F_{0}(\eta, b r) \\
u_{2}(r)=G_{0}(\eta, b r) .
\end{gathered}
$$

From Eqs.(7.27-28), and the Wronskian $F_{0} G_{0}^{\prime}-F_{0}^{\prime} G_{0}=b$ we obtain by simple differentiation the differential equation

$$
\gamma^{\prime}(r)=-W(r)\left[\cos \gamma(r) F_{0}(\eta, b r)+\sin \gamma(r) G_{0}(\eta, b r)\right]^{2} / b
$$

Note that for $W(r \rightarrow 0) \rightarrow \lambda(\lambda+1) / r^{2}$ (since $F_{0}(\eta, b r \rightarrow 0) \rightarrow C_{0} b r$ and $\left.G_{0}(\eta, b r \rightarrow 0) \rightarrow 1 / C_{0}\right)$, we obtain the relation

$$
\gamma^{\prime}(0)=-C_{0}^{2} b \lambda /(\lambda+1)
$$


Note that if we were to exclude the angular momentum barrier term $l(l+1) / r^{2}$ from $W(r)$ and include it in $\bar{W}(r)$ instead, $\lambda$ would become complex in the case of Eq.(7.3,7.6). With our division however, we must integrate the wave function to very large distances because of the long tail of the barrier term. However, we can overcome this difficulty by letting

$$
\gamma(r)=\beta(r)+\epsilon(r)
$$

with $\beta(r)$ defined so that

$$
\beta^{\prime}(r)=-l(l+1) / r^{2}\left[\cos \beta(r) F_{0}(r)+\sin \beta(r) G_{0}(r)\right]^{2} / b
$$

This equation has the exact solution

$$
\beta(r)=-\tan ^{-1}\left[\frac{F_{l}(r) F_{0}^{\prime}(r)-F_{l}^{\prime}(r) F_{0}(r)}{F_{l}(r) G_{0}^{\prime}(r)-F_{l}^{\prime}(r) G_{0}(r)}\right]
$$

with $\beta(0)=0$ and $\beta^{\prime}(0)=-C_{0}^{2} b l /(l+1)$ and

$$
\beta(\infty)=\sigma_{l}-l \pi / 2-\sigma_{0}
$$

Combining $(7.20,7.22,7.25,7.31,7.34)$ then leads to

$$
\delta_{l}=\epsilon(\infty)
$$

Thus, if we solve

$$
\begin{gathered}
\epsilon^{\prime}(r)=-\left(l(l+1) / r^{2}+\Delta \Phi\right) \\
\times\left[\cos \left(\beta(r)+\epsilon(r) F_{0}(\eta, b r)+\sin (\beta(r)+\epsilon(r)) G_{0}(\eta, b r)\right]^{2} / b\right. \\
+l(l+1) / r^{2}\left[\cos \beta(r) F_{0}(r)+\sin \beta(r) G_{0}(r)\right]^{2} / b
\end{gathered}
$$


subject to the boundary condition $\epsilon(0)=0$ and the condition Eq.(7.30), transcribed to the form $\epsilon^{\prime}(0)=-C_{0}^{2} b \lambda /(\lambda+1)+C_{0}^{2} b l /(l+1)$, we obtain the additional phase shift (above the Coulomb phase shift) by integration to $\epsilon(\infty)$.

As a first application of Eq.(7.36) we compute $\delta_{l}$ for the spin singlet equation in which

$$
\Delta \Phi=-\frac{\alpha^{2}}{r^{2}}
$$

This provides a particularly strong test of our procedure since $\delta$ can be computed analytically by incorporating the term $\frac{-\alpha^{2}}{r^{2}}$ with $\frac{l(l+1)}{r^{2}}$ into $\frac{\lambda(\lambda+1)}{r^{2}}$ and using

$\sigma_{l}-l \pi / 2=\arg \Gamma(l+1+i \eta)-l \pi / 2 \rightarrow \sigma_{\lambda}-\lambda \pi / 2=\arg \Gamma(\lambda+1+i \eta)-\lambda \pi / 2$

where $\lambda(\lambda+1)=l(l+1)-\alpha^{2}$. We are interested in the phase shift $\delta_{l}$ produced by $\Delta \Phi$ beyond the Coulomb phase shift $\sigma_{l}$ which in this case is given by

$$
\delta_{l}=\sigma_{\lambda}-\sigma_{l}
$$

Hence, with $\eta=-\frac{\alpha \epsilon_{w}}{b}$ we find

$$
\delta_{l}=\arg \frac{\Gamma(\lambda+1+1 \eta)}{\Gamma(l+1+i \eta)}-(\lambda-l) \pi / 2
$$

For $l=0,1$ and $w=1.6 \mathrm{MeV}$ we obtain $\delta_{l}=8.391 \cdot 10^{-5}, 2.794 \cdot 10^{-5}$. Using the first term in the perturbative expression (7.13) for comparison we obtain $\delta_{l}=8.431 \cdot 10^{-5}, 2.797 \cdot 10^{-5}$. The corresponding numerical results are $\delta_{l}=8.396 \cdot 10^{-5}, 2.770 \cdot 10^{-5}$. Thus we find agreement between the perturbative and numerically calculated values of the phase shifts for the singlet states. 
We are now ready to perform the same calculations and comparisons for the ${ }^{3} P_{0}$ states in order to test our Eq.(7.1) and to see whether it predicts any resonant states. We compute the results for the ${ }^{3} P_{0}$ states at several energies. At $w=1.4,1.6 .1 .8,2.0 \mathrm{MeV}$ we find that the perturbatively computed values using the full Eq.(7.13) are 2.556.10-3, 2.876 . $10^{-3}, 3.075 \cdot 10^{-3}$ and $3.210 \cdot 10^{-3}$. These agree well with the nonperturbatively computed numerical values $2.529 \cdot 10^{-3}, 2.847 \cdot 10^{-3}, 3.038 \cdot 10^{-3}$ and $3.175 \cdot 10^{-3}$. Thus our two-body Dirac equations predict no resonances in the ${ }^{3} P_{0}$ states in the above energy range, and no significant deviation from ordinary Bhabba scattering.

Let us examine now how the various parts of the quasipotential in Eq. (7.1) conspire to produce the turnover of the total effective potential (including the angular momentum barrier) for the ${ }^{3} P_{0}$ state while keeping the potential narrow and shallow enough to forbid a resonance (see Figs. 1a-b). At very long distances the Coulomb term dominates. As the interparticle separation goes to zero, the angular momentum barrier $\frac{l(l+1)}{r^{2}}\left(=\frac{2}{r^{2}}\right)$ becomes dominant at about an Angstrom. At this distance the spin-orbit and tensor terms (combining to give the next to last term in the first line) have an attractive $\frac{1}{r^{3}}$ behavior, whereas the spin-spin and Darwin terms (combining to give the last term in the first line) yield a repulsive $\frac{1}{r^{4}}$ behavior. The attractive $\frac{1}{r^{3}}$ terms counteract the angular momentum barrier reducing that barrier by about a factor of one-half for $r \sim 0.5 \mathrm{fm}^{66}$, eventually causing the potential to turn over at around $0.06 \mathrm{fm}$. But by this distance, the $G^{2}$ factor $\frac{1}{1+2 \alpha / w r}$ approaches $\frac{w r}{2 \alpha}$ so that it moderates the attractive $\frac{1}{r^{3}}$ spin-orbit part, leading to $=-\frac{4}{r^{2}}$. At about the same distance, the spin-spin and Darwin terms of the poten- 
tial have their repulsive $\frac{1}{r^{4}}$ behavior modified by the factor $G^{4}\left(\rightarrow \frac{w^{2} r^{2}}{4 \alpha^{2}}\right)$ to the form $\frac{2}{r^{2}}$. The net result is the $-\frac{\alpha^{2}}{r^{2}}$ behavior given by (7.1) for the ${ }^{3} P_{0}$ state. This behavior and the attendant phase shift are a direct consequence of the matrix $\Delta$ we obtained from QED and the minimal interaction structure for incorporating QED into our two-body Dirac equations $^{67}$. 


\section{Conclusions and Relationships to Other Approaches}

In this paper, we have solved a system of coupled Dirac equations previously formulated by two of us for electrodynamic and related two-body systems ${ }^{5,16}$. These equations, which are spin-dependent strong-potential versions of an equation originally developed for QED by Todorov ${ }^{14}$, contain local but non-singular potentials and so may be solved nonperturbatively for bona-fide relativistic wave functions. Yet, they contain effects in their wave functions that are traditionally obtained from perturbation theory. Two of us had previously found sixteen component exact analytic solutions for singlet states of positronium with energies agreeing with the field theoretic spectrum through order $\alpha^{4}$. In this paper, we have shown for a representative set of radial, orbital and spin states that nonperturbative numerical solutions for the wave function yield the correct field theoretic spectrum through order $\alpha^{4}$. As far as we know, this sort of spectral agreement has never been obtained before from numerical solution of a relativistic wave equation. Even though we had originally applied these equations (with appropriate potentials $\mathcal{A}, \mathcal{V}$ and $S$ ) to calculations of the meson spectrum, their suitability for electrodynamics is not a total surprise since two of us had originally abstracted the form of the vector interactions appearing in them from (the field theoretic) Wheeler-Feynman electrodynamics ${ }^{13}$.

Comparison of the structures of our equations with those of selected traditional approaches to QED and with those of recent alternatives and applications will help to clear up the origins and possible physical significance of our results. All of the equations that we will consider share the property that when treated perturbatively they reproduce the 
correct QED spectral results through order $\alpha^{4}$ that arise from the field theoretic Born diagram alone.

Relativistic wave equations have been used in electrodynamics primarily in three ways. First, such equations have been solved both numerically and analytically as wave equations when absence of singularities and non-local terms permitted leading to "nonperturbative" spectra (Balmer formulae). (However, such solutions are not guaranteed to agree with the results of quantum field theory - witness the erroneous results for parapositronium produced by nonperturbative treatment of the local Breit equation ${ }^{31-32}$.) Second, such equations, as they stand, have been used as perturbative forms that are divided into a nonrelativistic wave equation with well behaved solutions and a singular remainder to be used only in low order perturbation theory. Third, such equations have been used purely as spring boards for field theoretic perturbative treatments. Typically, one selects a relativistic wave equation with simple wave functions that generate the correct lowest order ( $\alpha^{2}$ and parts of the order $\alpha^{4}$ and higher order) spectrum directly through the wave function and then systematically treats the remaining order $\alpha^{4}$ and higher effects dictated by the Bethe-Salpeter equation as field theoretic perturbations built around the analytic solutions of the wave equation.

A. "Nonperturbative" Features of Wave Equations and Solution Where Possible.

Properly our numerical wave functions and spectra ought to be compared directly with their counterparts from numerical solution of the Bethe-Salpeter equation. However, as far as we know there have been no numerical tests (nonperturbative solutions) of any of the tradi- 
tional three-dimensional rearrangements of the four-dimensional BetheSalpeter equation of QED. (This situation has occurred because perturbative treatment of the weak potential forms of those equations (see B. below) are sufficient for QED and because treatment of non-local bound state equations has been technically difficult.) To see why this is so consider the most widely used rearrangement: the Salpeter equation ${ }^{17}$. That equation for single photon exchange in the instantaneous approximation is

$$
\left[E-H_{1}-H_{2}\right] \phi_{12}(\vec{p})=\Lambda(\vec{p}) \frac{e^{2}}{(2 \pi)^{3}} \int d^{3} k \frac{\gamma_{01} \gamma_{\mu 1} \gamma_{02} \gamma_{2}^{\mu}}{|\vec{k}|^{2}} \phi_{12}(\vec{p}+\vec{k})
$$

where $H_{1}(\vec{p})=m_{1} \beta_{1}+\vec{p} \cdot \vec{\alpha}_{1}, H_{2}(\vec{p})=m_{2} \beta_{2}-\vec{p} \cdot \vec{\alpha}_{2}, \Lambda=\left[\Lambda_{+}^{1}(\vec{p}) \Lambda_{+}^{2}(\vec{p})-\right.$ $\left.\Lambda_{-}^{1}(\vec{p}) \Lambda_{-}^{2}(\vec{p})\right], \Lambda_{ \pm}^{i}(\vec{p})=\left[E_{i}(\vec{p}) \pm H_{i}(\vec{p})\right] / 2 E_{i}(\vec{p})$, and $E_{i}(\vec{p})=\sqrt{m_{i}^{2}+\vec{p}^{2}}$ in which the three dimensional Salpeter wave function $\phi$ is given in terms of the four-dimensional Bethe-Salpeter wave function $\chi$ by $\phi_{12}(\vec{p})=$ $\int d p^{0} \chi_{12}\left(p^{0}, \vec{p}\right)$ and $\phi_{12}^{+-}(\vec{p})=\phi_{12}^{-+}(\vec{p})=0$ where $\phi_{12}^{\kappa \lambda}=\Lambda_{\kappa}^{1} \Lambda_{\lambda}^{2} \phi_{12}$, for $\kappa, \lambda:= \pm$. The particular (but ad hoc) elimination of the relative time and the relative energy ${ }^{68}$ in the derivation of Eq.(9.1) forces on the user non-local (in coordinate space) free-particle energies $E_{i}(\vec{p})$. In contrast, the corresponding role is played in our equations by the local but c.m. energy dependent $\epsilon_{i}$ of Todorov. Furthermore, the compatibility of our two sixteen component Dirac equations automatically restricts their dependence on the relative time in such a way as to permit an exact reduction (with no truncations) to two coupled (and in some angular momentum cases, one), Schrödinger-like equations with a total c.m. energy dependent (but not necessarily) momentum-dependent effective potential, each involving two four-component wave functions (see Eqs.(4.21a-b)). Not 
only are our local minimal interaction constraint equations much easier to handle numerically, but also, (for momentum-independent interactions), they permit a direct covariant examination of the short distance behavior (see Figs. 1a-b). Such an examination cannot be performed as directly on the momentum space form or on the necessarily non-local coordinate space form of the three-dinensional Salpeter equation without an $O\left(1 / c^{2}\right)$ expansion (which we shall examine below).

Recently, Mandelzweig and Wallace ${ }^{60}$ have presented a new covariant approach to the two-body protlem. Instead of the two two-body Dirac equations of $(2.1 \mathrm{a}-\mathrm{b})$ or $(3.38 \mathrm{a}-\mathrm{b})$ they employ a single sixteen component "sum" Dirac equation of the form

$$
\left[\left(\gamma_{1} \cdot p_{1}+m_{1}\right) \lambda_{1}+\left(\gamma_{2} \cdot p_{2}+m_{2}\right) \lambda_{2}-V\right] \psi=0
$$

(in our metric) in which

$$
\lambda_{i}=\frac{\left(\gamma_{i} \cdot p_{i \perp}+m_{i}\right)}{\sqrt{m_{i}^{2}+p_{i \perp}^{2}}}
$$

accompanied by a spin-independent constraint

$$
\left(p_{1}^{2}+m_{1}^{2}\right) \psi=\left(p_{2}^{2}+m_{2}^{2}\right) \psi
$$

on the relative energy. (In our approach Eq.(9.4) is a consequence of our two Dirac equations (see (3.21) and also Refs.(4-5)).)

Like our equations, the Mandelzweig-Wallace equations yield the correct single particle Dirac equation with an external potential when one particle becomes infinitely heavy. However, each set of equations achieves this result in different way. Mandelzweig and Wallace noted that in the Bethe-Salpeter formalism both the single particle Dirac limit and the 
high energy Eikonal limit depend on cancellations between crossed and uncrossed Feynman graphs when the kernel in the Bethe-Salpeter equation is truncated. Consequently, in deriving their equation, they included crossed graphs using a form of the Eikonal approximation in such a way that the high energy limit and the heavy particle limits are preserved despite truncation of the kernel. In contrast our spin-dependent equations and the closely related spinless Todorov quasipotential equation (in the form (3.1)) achieve the heavy particle limits automatically through their classical relativistic kinetic and potential structures without further manipulation of the potential. (In fact starting from only the Born term, the Todorov equation sums up all cross ladder and ladder diagrams in the limit of small exchanged mass and momentum transfer ${ }^{14,70}$.)

The two-body Dirac equations Eqs.(2.1a-b) and the MandelzweigWallace equation differ substantially in their spin-dependent structures through the full $16 \times 16$ matrix potentials. Just like the free particle sub-energies $E_{i}(\vec{p})$ of the Salpeter equation, the Mandelzweig-Wallace equation contains the free particle Dirac projectors $\lambda_{i}$ (which contain the $\left.E_{i}(\vec{p})\right)$ as coefficients in the "sum" form Eq.(9.2). These render the Mandelzweig-Wallace equation non-local in the Born approximation, for which the coupled Dirac equations (Eqs.(2.1a-b) or Eqs.(4.21a-b)) are local. Nonetheless Wallace and Thayyullathil (Ref.33) have been able to solve the Mandelzweig-Wallace equations numerically for the ground state hyperfine splittings in QED. Thus, some results given by both sets of equations are available for comparison. These results show that one consequence of the difference in spin-dependent structures in the two approaches is their different dependences on and sensitivities to the four 
four-component pieces of the sixteen component wave function. For example, for the hyperfine splitting of muonium, the Mandelzweig-Wallace results with the lower-lower parts of the wave function excluded are comparable to our results through order $\alpha^{4}$ when our equations are fully coupled. However, inclusion of the lower-lower portion of the wave function in the Mandelzweig-Wallace equation produces large deviations from the field theoretic values through order $\alpha^{4}$. This contrasts sharply with our results of section VI for which inclusion of the coupling to the lowerlower portion of the wave function was crucial for agreement with the field theoretic values through order $\alpha^{4}$. A complete comparison of the two approaches awaits calculations in the Mandelzweig-Wallace approach of the counterparts to the fine-structure splitting and radial excitations given by our equations in section VI.

\section{B.) Weak-Potential Perturbative Form.}

Traditionally relativistic wave equations which cannot be or have not been solved numerically or analytically have been rearranged as corrections to the non-relativistic Schrödinger equation with Coulomb potential. For example the $O\left(1 / c^{2}\right)$ Fermi-Breit expansion $^{71}$ of the Salpeter equation, yields

$$
H \psi=w \psi
$$

in which $w$ is the total c.m. energy and

$$
\begin{aligned}
& H=\left(m_{1} c^{2}+\frac{\vec{p}^{2}}{2 m_{1}}-\frac{\left(\vec{p}^{2}\right)^{2}}{8 m_{1}^{3} c^{2}}\right)+\left(m_{2} c^{2}+\frac{\vec{p}^{2}}{2 m_{2}}-\frac{\left(\vec{p}^{2}\right)}{8 m_{2}^{3} c^{2}}\right)+ \\
& e_{1} e_{2}\left(\left[\left(1-\frac{p^{2}}{m_{1} m_{2} c^{2}}\right) \frac{1}{r}-\frac{1}{2 m_{2} m_{2} c^{2} r} \vec{p} \cdot(1-\hat{r} \hat{r}) \cdot \vec{p}\right]_{\text {ordered }}\right.
\end{aligned}
$$




$$
\begin{aligned}
& -\frac{\hbar^{2}}{2 c^{2}}\left(\frac{1}{m_{1}^{2}}+\frac{1}{m_{2}^{2}}\right) \delta(\vec{r})-\frac{\hbar}{4 c^{2}} \frac{\vec{L}}{r^{3}} \cdot\left[\left(\frac{1}{m_{1}^{2}}+\frac{2}{m_{1} m_{2}}\right) \vec{\sigma}_{1}+\left(\frac{1}{m_{2}^{2}}+\frac{2}{m_{1} m_{2}}\right) \vec{\sigma}_{2}\right] \\
& \left.\quad+\frac{\hbar^{2}}{4 m_{1} m_{2} c^{2}}\left(-\frac{8 \pi}{3} \vec{\sigma}_{1} \cdot \vec{\sigma}_{2} \delta(\vec{r})+\frac{\vec{\sigma}_{1} \cdot \vec{\sigma}_{2}}{r^{3}}-\frac{3 \vec{\sigma}_{1} \cdot \vec{r} \vec{\sigma}_{2} \cdot \vec{r}}{r^{5}}\right)\right)
\end{aligned}
$$

This equation (to which our equation is canonically equivalent in order of $O\left(1 / c^{2}\right)$ for weak potentials $\left.{ }^{72}\right)$ contains terms after the Coulomb term that are too singular at the origin to be treated non-perturbatively. On the other hand, the unapproximated counterparts of these terms in the covariant, Schrödinger-like form Eqs.(4.21a-b) of the two-body Dirac equations are quantum mechanically well defined for all tested angular momentum states. This means that in contrast to the Fermi-Breit equation (and Eq.(5.9)), the wave functions for all angular momentum states are affected by all terms. Despite this fact, our solution of the unapproximated covariant minimal interaction constraint form, (4.21a-b), reproduces the correct perturbative spectral results for fine and hyperfine splittings. Because we are able to solve our unapproxinated equation numerically, we are able to carry out a double cross check of its nonperturbative spectral results with its own perturbative spectral results (from Eq.(5.9)) and with the corresponding results of percurbative quantum field theory (from Eq.(9.5a-b)). The fact that they all agree shows that: i.) The weak potential form Eq.(5.9) yields an accurate perturbative evaluation of the exact equation Eqs.(4.21a-b) (mathematical property). ii.) The unusual (though local) short distance structure of the exact equation Eqs.(4.21a-b) (and hence of the wave function) does not disrupt the perturbative spectrum (mathematical property). iii.) The unapproximated coupled two-body Dirac equations Eqs.(2.1a-b) and their Schrödingerlike rearrangement Eqs.(4.21a-b) yield a spectrum from single photon 
exchange in agreement with that of perturbative QED for all tested angular momentum states through order $\alpha^{4}$ (physical property). Although this agreement exists in the one-body Dirac equation, as far as we know because of difficulty of nonperturbative solution, such agreement has never been demonstrated for any other two-body equation with spin. ${ }^{73-74}$

C. Relativistic Wave Equations as Anchors for Field Theoretic Perturbation Theury

In order to carry out any perturbative solution of the Bethe-Salpeter equation of QED, one must first specify a lowest order equation. As noted by Barbieri and Remiddi ${ }^{75}$, any such equation must contain a kinetic term, a Coulomb-like interaction term, be able to produce bound states, and contain the largest part of the full Bethe-Salpeter kernel. It must reproduce the correct nonrelativistic dynamics with corrections of second order in momenta so that the usual Balmer formula appears in lowest order $\left(\alpha^{2}\right)$ with no corrections of order $\alpha^{3}$. In addition, it must yield the relativistic propagator of two free fermions when the interaction vanishes (at high momer um). Finally, for purposes of perturbative calculation, its wave functions must be known analytically or numerically. By carrying out the numerical solution of the two-body Dirac equations Eqs.(2.1a-b) in this paper, we have completed the demonstration that they possess all these properties.

In the work of Barbieri and Remiddi ${ }^{75}$ and in the work of Caswell and Lepage ${ }^{35}$ (for fermions of comparable mass), the fact that "no equation for two fermions is known that can be solved exactly and which gives the correct $\alpha^{4}$ structure" forced those authors to confine the dynamical contributions of their lowest order equations to the relativistic Coulomb 
potential alone. This restriction does not destroy the perturbative procedure, however, as long as "the correct $\alpha^{4}$ terms, as well as all higher order corrections to the energy levels, are obtained in the systematic perturbative expansion to be built starting from the lowest order equation in question."

Why then would anyone propose to replace the basic relativistic wave equation with Coulomb potential by an equation with additional dynamical structures? The advantages are twofold. First, in most mathematical structures, increase in accuracy of the unperturbed piece pays dividends in the form of increased rate of convergence and the ability to dispense with the treatment of terms whose only function is to build up some basic structure of the unperturbed term. If one includes a basic nonperturbative structure, one gets all of the higher order perturbative terms corresponding to it as a bonus. For example, Barbieri and Remiddi, and Caswell and Lepage pass from the nonrelativistic Schrödinger equation with nonrelativistic Coulomb potential to a relativistic wave equation with relativistic potential to reap the benefit of inclusion of some of the $\alpha^{4}$ terms in their lowest order equation and to ensure correct relativistic kinematical contributions in higher order. In addition, Caswell and Lepage, in their first work on systems with one heavy particle, one light particle took as their lowest order equation the one-body Dirac equation with second particle on the mass shell in order to include fine-structure (i.e. Dirac spin structure) in their unperturbed solutions. Second, the lowest order equation of perturbative QED with its particular structures is abstracted by many authors for use elsewhere in QED, nuclear, and particle physics as a wave equation for bound state 
calculations. In those applications structures of little consequence in perturbative QED (e.g. short distance or strong potential behaviors) may play a significant role. Thus, accurate knowledge of QED structures of this sort serves as a check on the uses and abuses of such equations.

As we have mentioned, as first shown by Yaes and Gross ${ }^{17}$, there is an infinite set of "equivalent" three-dimensional reductions of the BetheSalpeter eqiation, differing in form - therefore in ease of application and interpretation. The electromagnetic constraint equation employed in this paper, with its characteristic energy-dependences and potential structures permits nonperturbative solution to higher-order in $\alpha$ than has yet been possible for others in the set. To illustrate this point, we consider in some detail the work of Caswell and Lepage ${ }^{35}$. Caswell and Lepage reformulated the Bethe-Salpeter equation in two different ways the first in terms of a one-body Dirac equation with the second particle on the mass-shell, - the second in terms of an effective Schrödinger equation (in c.m. frame). In the first approach Caswell and Lepage were able to incorporate "fine-structure of levels with differing angular momenta" in the unperturbed QED solutions. However, the unsymmetric nature of this solution restricted its application to cases in which the binding was weak or the mass-ratio large. To remedy this defect, Caswell and Lepage developed an effective Schrödinger equation to treat the case of comparable masses. The price they had to pay was loss of unperturbed solutions containing fine-structure of levels with differing angular momenta. They were able only to retain their version of a relativistic Coulomb potential in their unperturbed Schrödinger equation. They at- 
tributed the different structures of their treatments to the physical fact that "the fine-structure of atoms with constituents of equal mass is quite different in character from that of atoms with a large mass-ratio." They observed that "it is difficult to create a formalism which naturally accommodates both cases and still admits analytic solutions comparable in simplicity to those presented" (in their second paper).

However, the electromagnetic two-body Dirac equations Eqs.(4.21ab) are a solution to the problem posed by Caswell and Lepage. Since these describe the system symmetrically through two coupled Dirac equations whose potential structures do not change discontinuously from high mass-ratio to comparable masses, they can be solved nonperturbatively in both regimes using the same numerical techniques. Furthermore, for comparable masses, they can be solved numerically without truncaticn to the simple Coulomb potential of Caswell and Lepage. Thus, their solutions contain the fine-structure lost by Caswell and Lepage.

We may see this explicitly by making use of the fact that in one case - the equal-mass singlet - our equations possess an exact analytic solution (see Ref.16 and appendix D). In fact, in that case, the secondorder form of our equations reduces to the minimal Todorov equation ${ }^{76}$ on a singlet wave function:

$$
\left[\vec{p}^{2}+2 \epsilon_{w} \mathcal{A}-\mathcal{A}^{2}-\epsilon_{w}^{2}+m_{w}^{2}\right] \psi=0
$$

in which $\epsilon_{w}$ and $m_{w}$ are Todorov's reduced energy and mass of a relativistic particle of relative motion introduced so that the second order two-body equation takes the mass-shell form. On the other hand, Caswell and Lepage write their second order equation in the form of an effective 
non-relativistic Schrödinger equation:

$$
\left[\frac{\tilde{p}^{2}}{2 \tilde{m}}+\mathcal{A}\right] \psi=\tilde{\epsilon} \psi
$$

in which $\tilde{e}$ and $\tilde{m}$ are an effective binding energy and mass given by

$$
\tilde{\epsilon}=\frac{w^{2}-\left(m_{1}+m_{2}\right)^{2}}{2 w}, \quad \tilde{m}=\frac{w^{2}-\left(m_{1}-m_{2}\right)^{2}}{2 w}
$$

In fact, we see that $\tilde{\epsilon}$ is simply the difference

$$
\tilde{\epsilon}=\epsilon_{w}-m_{w}
$$

while $\tilde{m}$ is the average

$$
\tilde{m}=\frac{1}{2}\left(\epsilon_{w}+m_{w}\right)
$$

Using these facts, we may rewrite the Caswell-Lepage equation in the Todorov form:

$$
\left[\vec{p}^{2}+\left[\epsilon_{w}+m_{w}\right] \mathcal{A}-\epsilon_{w}^{2}+m_{w}^{2}\right] \psi=0
$$

We see that for the singlet state, the unperturbed equation that we solve shares its relativistic kinetic structure with that of Caswell and Lepage. On the other hand, the two equations differ in their dependence on the relativistic Coulomb potential and on the energy-dependent $\epsilon_{w}$ and $m_{w}$. Despite its more elaborate structure, the minimal Todorov equation still permits exact solution ${ }^{16}$. In each case, the unperturbed eigenvalue may be found by mapping the relativistic equation to the non-relativistic Schrödinger equation with Coulomb potential $(\mathcal{A}=-\alpha / r)$. Rewritten in 
the Todorov variables, the Caswell-Lepage "relativistic Balmer formula" takes the form:

$$
\epsilon_{w}^{2}-m_{w}^{2}=-\frac{\alpha^{2}}{n^{2}} \tilde{m}^{2}
$$

whereas the minimal Todorov equation's "relativistic Balmer formula" is:

$$
\epsilon_{w}^{2}-m_{w}^{2}=-\frac{\alpha^{2}}{\left(n-\delta_{l}\right)^{2}} \epsilon_{w}^{2}
$$

in which $\delta_{l}$ is the relativistic shift of the angular momentum given by:

$$
\delta_{l}=l+1 / 2-\left[(l+1 / 2)^{2}-\alpha^{2}\right]^{1 / 2} .
$$

Solution of each of these for the total energy $w$ followed by expansion in $\alpha$ leads to

$$
w \sim 2 m-\frac{\alpha^{2} m}{4 n^{2}}+\frac{3}{64} \frac{\alpha^{4} m}{n^{4}}
$$

for the Caswell-Lepage equation and

$$
w \sim 2 m-\frac{\alpha^{2} m}{4 n^{2}}-\frac{\alpha^{4} m}{2 n^{3}(2 l+1)}+\frac{11}{64} \frac{\alpha^{4} m}{n^{4}}
$$

for the minimal Todorov equation, respectively. Note that the singlet eigenvalue for our equation (the minimal Todorov equation) already contains the correct angular-momentum-dependent fine-structure as well as the correct angular-momentum-independent fine-structure correct through order $\alpha^{4}$. On the other hand, Caswell and Lepage's unperturbed fine-structure must be perturbatively corrected by "relativistic corrections to single Coulomb exchange" and "single transverse photon exchange" in the Coulomb gauge to yield the singlet spectrum correct to order $\alpha^{4}$. 
Now that we have a new lowest order equation, how are we to go on to higher order perturbation theory? One could use that equation for a new approach to perturbative QED calculations for bound states. We have shown in Ref.2, appendix A and in section III how one may use the projection of Sazdjian to obtain Todorov's inhomogeneous quasipotential equation from the Bethe-Salpeter equation. One could use that equation to correct the interactions that appear in the two-body Dirac equations perturbatively, and then one could solve the resulting corrected wave equation nonperturbatively just as we solved the lowest order equation in this paper. (This would avoid the necessity of using higher order quantum mechanical perturbation theory.)

D. Nonperturbative Application of Relativistic Wave Equations to $e^{+} e^{-}$and $q \bar{q}$ Composites.

In the past, many authors have transported the relativistic wave equations and relativistic correction structures of perturbative electrodynamics far from their origins in perturbation theory. In the process, strong potential structures of these equations which were of no consequence (to a given order) in perturbation theory may come to play an important role. This has several consequences. First, equations whose agreement with quantum field theory has been checked perturbatively but not when solved as wave equations may be used in the (sometimes mistaken) belief that solution works. The danger of this is illustrated by the local Breit equation whose nonperturbative treatment produces erroneous results for parapositronium and which, as has been pointed out by Childers, leads to singular potentials for other $e^{+} e^{-}$states $^{77}$. The 
agreement must be checked, as we have done for two-body Dirac equations Eqs.(4.21a-b) in section VI. Second, different wave equations that gave equivalent results when treated perturbatively (to a given order) may yield inequivalent results when solved nonperturbatively.

As we have shown, the two-body Dirac equations Eqs.(2.1a-b) and Eqs.(4.21a-b) provide an alternative treatment of the two fermion bound state problem. These equations use their own characteristic local potential structures to produce the same spectra for perturbative QED that are produced by more complicated momentum structures in standard equations. Thus, when such equations are extended to other problems, we may find disagreements of the first or second types. For their part, the two-body Dirac equations yield straightforward numerical solutions for (eventual) comparison with the other methods.

Recently, motivated by the work ${ }^{18}$ of two of us, Spence and Vary ${ }^{28}$ have nonperturbatively solved three different three dimensional truncations of the Bethe-Salpeter equation with single photon exchange and in each instance obtained "zero width $e^{+} e^{-}$resonances" (continuum bound states) at 1.351,1.498,1.659,1.830,2.009, and $2.195 \mathrm{MeV}$ in direct contradiction to our results of section VII. The reader should note that Spence and Vary restrict themselves to the same field theoretic dynamics (single photon exchange) that we do. They use the Tamm-Dancoff equation, the no-pair form of the Breit equation (the Salpeter equation), and the Blankenbecler-Sugar equation along with a nonperturbative treatment of the corresponding Lippmann-Schwinger equations. All of the equations that they employ are nonlocal; they claim that this feature is crucial for generating their continuum bound state solutions. They point out 
that one of these equations, - the no pair form of the Breit equation is known to produce a good description of the ordinary bound states of positronium. However, the bound state calculation that they refer to is actually a perturbative calculation and thus lends no support to their nonperturbative solution of that equation. Thus, if one were to trust their results, one would first have to rule out a disagreement of the first type by carrying out a nonperturbative treatment of each of these truncations to obtain the standard QED energy levels through order $\alpha^{4}$ (just as we have done for Eqs.(4.21a-b) in section VI). Interestingly, we were originally motivated to study the nonperturbative treatment of the QED bound state spectra in our equation by the possibility that the potential structures responsible for disagreements of the second type could lead to highly relativistic resonant $e^{+} e^{-}$states.

Thus far there is no direct evidence for $e^{+} e^{-}$resonances in Bhabha scattering experiments. Recent searches for both short lived and long lived low mass couplings in the $e^{+} e^{-}$system have found no evidence for deviations from the nonresonant Bhabha scattering background within statistical uncertainties of $0.2 \%(\sigma)$ in the invariant energy range from 1500 to $1850 \mathrm{keV}$. Of course if the authors of Ref.(28) calculate corrections to their zero width predictior:s, they may find lifetimes outside the range looked for in the experiments. On the other hand, if our treatment of this problem resembles the full Bethe-Salpeter solution, such states do not exist, so that one must look beyond the two particle sector of pure QED to explain the relativistic resonances seen in heavy ion collisions.

In yet another area of relativistic two-body physics, various authors have borrowed (sometimes innocently and sometimes with additional 
cutoffs $^{78}$ ) interactions from nonrelativistic and relativistic electrodynamics for use in models of quark-antiquark bound systems. In previous papers $^{1-2}$, two of us have applied two-body Dirac equations with potential structures motivated by QCD to calculate the mass spectra of mesons composed of light quarks along with those composed of heavy quarks. The goodness of the resulting fit to the full meson spectrum was due in no small part (especially for the "hyperfine" and "fine-structure" splittings), to the peculiar short distance vector interaction structure of our equations inherited from both the constituent and collective (Todorov) minimal interaction structures contained within them. As we have shown, this structure, when applied to electrodynamics itself, reproduces the two-body spectrum of QED. These results, taken together, argue that any competing approach to QCD which solves wave equations in which short distance dynamics is dominated by effective abelian replacements for the Coulomb potential inserted into elaborations of the Darwin interaction (or equivalently the Breit interaction) or which are based on truncations of the Bethe-Salpeter equation of QED should be judged on their ability to reproduce the spectra of QED when treated numerically or analytically before being applied to QCD. Measures to avoid singularities in the interactions borrowed from certain approaches to $Q E D$, such as the use of cutoffs, may invalidate the equations for QED applications, introducing spurious dynamics. 


\section{Appendices}

\section{A- Derivation of Todorov's Inhomogeneous Quasipotential Equa- tion for Spinless Particles from The Bethe Salpeter Equation}

Written in terms of the constituent c.m. energies $\epsilon_{1}, \epsilon_{2}$, the inhomogeneous Bethe-Salpeter equation in an aribitrary Lorentz frame takes the form

$T_{w}(p ; q)=K_{w}(p ; q)-\frac{i}{(2 \pi)^{4}} \int d^{4} k K_{w}(p ; k) G_{1}^{(+)}\left(\epsilon_{1} \hat{P}+k\right) G_{2}^{(+)}\left(\epsilon_{2} \hat{P}-k\right) T_{w}(k ; q)$

or symbolically,

$$
T_{w}=K_{w}+K_{w} G_{12, w}^{(+)} T_{w}, \quad G_{12, w}^{(+)}=-i(2 \pi)^{-4} G_{1}^{(+)} G_{2}^{(+)} .
$$

Eq.(A.1) relates the off mass-shell scattering amplitude $T_{w}(p ; q)$ to the two-particle-irreducible kernel $K_{w}(p ; q) .\left(G_{i}^{(+)}\right.$is the Feynman propagator.) For an incident free (on mass-shell) plane wave, given in relative momentum space by

$$
\tilde{\chi}_{w, q_{\perp}}^{(0)}(p)=\delta(\hat{P} \cdot p) \delta^{3}\left(p_{\perp}-q_{\perp}\right)
$$

we construct the Bethe-Salpeter "wave function" $\tilde{\chi}_{w, q_{\perp}}^{(+)}$

$$
T_{w}\left(p ; q_{\perp}\right)=\int d^{4} p^{\prime} T_{w}\left(p ; p^{\prime}\right) \tilde{\chi}_{w, q_{\perp}}^{(0)}\left(p^{\prime}\right) \equiv \int d^{4} p^{\prime} K_{w}\left(p ; p^{\prime}\right) \tilde{\chi}_{w, q_{\perp}}^{(+)}\left(p^{\prime}\right)
$$

in which $q=-\hat{P}(\hat{P} \cdot q)+q_{\perp}$. Then (A.1) is reproduced for $\hat{P} \cdot q=0$, if

$$
\tilde{\chi}_{w, q_{\perp}}^{(+)}\left(p^{\prime}\right)=\delta\left(\hat{P} \cdot p^{\prime}\right) \delta^{3}\left(p_{\perp}^{\prime}-q_{\perp}\right)+G_{12, w}^{(+)}\left(p^{\prime}\right) T_{w}\left(p^{\prime} ; q_{\perp}\right)
$$




$$
=\tilde{\chi}_{w, q_{\perp}}^{(0)}\left(p^{\prime}\right)+G_{12, w}^{(+)}\left(p^{\prime}\right) \int d^{4} k K_{w}\left(p^{\prime} ; k\right) \tilde{\chi}_{w, q_{\perp}}^{(+)}(k) .
$$

In this equation $K_{w}$ plays the role of the potential (the role played by $\tilde{\mathbf{V}}_{w}$ in (3.12)). We now write the two-particle, off-mass shell, Feynman propagator as a sum,

$$
G_{12, w}^{(+)}(p)=\mathcal{G}_{w, f}^{(+)}(p)+R_{w, f}^{(+)}(p)
$$

of a "minimally off the mass shell" Green function

$$
\mathcal{G}_{w, f}^{(+)}(p)=\frac{1}{(2 \pi)^{4}} \frac{\pi}{w} \frac{f\left(p_{\perp}^{2}, w\right) \delta(\hat{P} \cdot p)}{\left(p_{\perp}^{2}-b^{2}(w)-i \epsilon\right)} \equiv \delta(\hat{P} \cdot p) G_{w, f}^{(+)}\left(p_{\perp}\right)
$$

and a residual, $R$. Like the Todorov Green function, $G_{w, f}^{(+)}$satisfies elastic unitarity provided that $f\left(p_{\perp}^{2}, w\right)=1$ on mass shell. $G_{w, f}^{(+)}$reduces to the Todorov Green function when $f=1$. For equal masses we obtain the Logunov-Tavkhelidze Green function when $f=w\left(2 \sqrt{p_{\perp}^{2}+m^{2}}+\right.$ $w) /\left(8\left(p_{\perp}^{2}+m^{2}\right)\right)$ and Blankenbecler and Sugar Green function when $f=w / 2 \sqrt{p_{\perp}^{2}+m^{2}}$. Following the work of Blankenbecler and Sugar ${ }^{43}$ we write $T$ in terms of the Green function $\mathcal{G}_{w, f}^{(+)}$and an effective interaction $W$ defined by

$$
\begin{gathered}
W_{w}=K_{w}+K_{w} R_{w} W_{w}=K_{w}+W_{w} R_{w} K_{w} \\
K=\left(1-K_{w} R_{w}\right) W_{w}=W_{w}\left(1-R_{w} K_{w}\right)
\end{gathered}
$$

Then (A.2), (A.6), and (A.7) imply that

$$
(1-K R) T=K+K \mathcal{G}_{w, f}^{(+)} T
$$

which is satisfied, according to (A.8b), when

$$
T=W+W \mathcal{G}_{w, f}^{(+)} T .
$$


Eq. (A.8a) does not restrict elements of $W$ to the mass shell. We wish to determine the quasipotential $V(=2 w \Phi)$ in terms of $W$. We begin by performing a general Sazdjian projection ${ }^{40,2}$ of the Bethe-Salpeter wave function $\tilde{\chi}^{(+)}$

$$
\phi_{w, f, q_{\perp}}^{(+)}(p)=\tilde{\chi}_{w, q_{\perp}}^{(0)}(p)+\delta(\hat{P} \cdot p) G_{w, f}^{(+)}\left(p_{\perp}\right) \int d^{4} k K_{w}\left(p_{\perp} ; k\right) \tilde{\chi}_{w, q_{\perp}}^{(+)}(k)
$$

This removes the c.m. relative energy dependence of $\tilde{\chi}_{w, q_{\perp}}^{(+}(p)$, so that like the constraint wave function, $\phi$ satisfies $\hat{P} \cdot p \phi_{w, f, q_{\perp}}^{(+)}(p)=0$. Then, we define the wave function $\tilde{\Psi}$ such that

$$
\phi_{w, f, q_{\perp}}^{(+)}(p)=\delta(\hat{P} \cdot p) \tilde{\Psi}_{w, f, q_{\perp}}^{(+)}\left(p_{\perp}\right)
$$

We can eliminate the general factor $f$ by multiplying Eq.(A.11) by $f^{-1}$ and using the fact that $f^{-1} \tilde{\chi}_{w, q_{\perp}}^{(0)}(p)=\tilde{\chi}_{w, q_{\perp}}^{(0)}(p)$. This produces the Todorov wave function

$$
\phi_{w, q_{\perp}}^{(+)}(p) \equiv f\left(p_{\perp}^{2}, w\right)^{-1} \phi_{w, f,{ }_{\perp}}^{(+)}(p) \equiv \delta(\hat{P} \cdot p) \tilde{\Psi}_{w, q_{\perp}}^{(+)}\left(p_{\perp}\right)
$$

Hence we see that all the Sazdjian wave functions $\phi_{w, f, q_{\perp}}^{(+)}(p)$ (and associated Green functions) are related to the Todorov choice $\phi_{w, q_{\perp}}^{(+)}(p)$ by a scale transformation. Todorov's choice, the simplest one $(f=1)$, yields the Schrödinger-like equation of constraint dynamics; hence we use it in this paper. We use (A.5) and (A.6) to rewrite the transform as

$$
\phi_{w, q_{\perp}}^{(+)}(p)=\int d^{4} k\left[\delta(p-k)-R_{w}^{(+)}(p) K_{w}(p ; k)\right] \tilde{\chi}_{w, q_{\perp}}^{(+)}(k)
$$

From (A.8b) and (A.14) we obtain, without employing formal inverses,

$$
\int d^{4} k K_{w}(p ; k) \ddot{\chi}_{w, q_{\perp}}^{(+)}(k)=\int d^{4} k^{\prime} W_{w}\left(p ; k^{\prime}\right) \phi_{w, q_{\perp}}^{(+)}\left(k^{\prime}\right)
$$




$$
=\int d^{3} k_{\perp}^{\prime} W_{w}\left(p ; k_{\perp}^{\prime}\right) \tilde{\Psi}_{w, q_{\perp}}^{(+)}\left(k_{\perp}^{\prime}\right)
$$

so that (A.11) can be written as

$$
\tilde{\Psi}_{w, q_{\perp}}^{(+)}(p)=\delta^{3}\left(p_{\perp}-q_{\perp}\right)+G_{w}^{(+)}\left(p_{\perp}\right) \int d^{3} k_{\perp} W_{w}\left(p_{\perp} ; k_{\perp}\right) \tilde{\Psi}_{w, q_{\perp}}(k)
$$

Eq.(A.16) has the same form as the quantum mechanical momentum space integral equation (3.12) for the constraint wave function. If we rewrite Eq.(A.8a) in terms of

$$
\tilde{V}_{w}\left(p_{\perp}, k_{\perp}\right)=-W_{w}\left(p_{\perp}, k_{\perp}\right)
$$

then

$$
\tilde{V}_{w}\left(p_{\perp}, k_{\perp}\right)+K_{w}\left(p_{\perp} ; k_{\perp}\right)+\int d^{4} k^{\prime} K_{w}\left(p_{\perp} ; k^{\prime}\right) R_{w}\left(k^{\prime}\right) W_{w}\left(k^{\prime} ; k_{\perp}\right)=0
$$

so that (A.10) implies the Todorov quasipotential equation

$$
\begin{gathered}
T_{w}\left(p_{\perp}, k_{\perp}\right)+\tilde{V}_{w}\left(p_{\perp}, k_{\perp}\right) \\
+\int \frac{d^{3} k_{\perp}^{\prime}}{(2 \pi)^{3}} \tilde{V}_{w}\left(p_{\perp}, k_{\perp}^{\prime}\right) \frac{1}{2 w\left(k_{\perp}^{\prime}{ }^{2}-b^{2}(w)-i \epsilon\right)} T_{w}\left(k_{\perp}^{\prime}, k_{\perp}\right)=0 .
\end{gathered}
$$

The difference between this field-theoretic equation and the formally equivalent quantum mechanical Lippmann-Schwinger Eq.(3.16) is that Eq.(A.19) gives $\tilde{V}_{w}$ in terms of $T_{w}$ whereas (3.16) gives $\mathbf{T}_{w}$ in terms of $\tilde{\mathbf{V}}_{w}$. Since the homogeneous form of Eq.(A.19) would be identical in form to the constraint equation Eq.(3.1) (with $\Phi=V / 2 w$ ) we use the field theoretic $\tilde{V}_{w}$ as the $\tilde{\mathbf{V}}_{w}$ in our quantum constraint equation.

As a simple example, consider a scalar Yukawa field theory with a momentum space Born amplitude

$$
T_{P}^{(1)}\left(p^{\prime}, q^{\prime}\right)=\delta\left(p_{1}^{\prime}+p_{2}^{\prime}-q_{1}^{\prime}-q_{2}^{\prime}\right) T_{w}^{(1)}\left(p^{\prime}, q^{\prime}\right) .
$$


Fence, the corresponding mornentum form of our constraint potential is

$$
\tilde{\mathbf{V}}_{w}^{(1)}\left(p_{\perp}^{\prime}, q_{\perp}^{\prime}\right)=-T_{w}^{(1)}\left(p_{\perp}^{\prime}, q_{\perp}^{\prime}\right)=-\frac{g_{1} g_{2}}{\left(p_{\perp}^{\prime}-q_{\perp}^{\prime}\right)^{2}+\mu^{2}-i 0}
$$

so that the coordinate space form of $\Phi_{w}$, the quasipotential to be used in our constraint equation (3.1), would be

$$
\Phi_{w}^{(1)}\left(x_{\perp}\right)=\frac{V_{w}\left(x_{\perp}\right)}{2 w}=-\frac{g_{1} g_{2}}{8 \pi w} \frac{e^{-\mu\left|x_{\perp}\right|}}{\left|x_{\perp}\right|}=-2 m_{w} \alpha \frac{e^{-\mu\left|x_{\perp}\right|}}{\left|x_{\perp}\right|}
$$

in which $g_{1} g_{2}=16 \pi m_{1} m_{2} \alpha$ and $m_{w}=\frac{m_{1} m_{2}}{w}$. In order to determine the constraint potential to a higher order (say $\mathbf{V}^{(2)}$ ) we would first have to evaluate the corresponding single loop diagrams $T_{w}^{(2)}$ (appropriately renormalized). In that case (A.19) leads to

$$
\begin{gathered}
\tilde{\mathbf{V}}_{w}^{(2)}\left(p_{\perp}, k_{\perp}\right)=-T^{(2)} w\left(p_{\perp}, k_{\perp}\right) \\
+\int \frac{d^{3} k_{\perp}^{\prime}}{(2 \pi)^{3}} \tilde{\mathbf{V}}_{w}^{(1)}\left(p_{\perp}, k_{\perp}^{\prime}\right) \frac{1}{2 w\left(k_{\perp}^{\prime 2}-b^{2}(w)-i \epsilon\right)} \tilde{\mathbf{V}}_{w}^{(1)}\left(k_{\perp}^{\prime}, k_{\perp}\right)
\end{gathered}
$$


B Direct Derivation of Eq.(4.12) from First Order For $\mathrm{m}$ of the Two-Body Dirac Equations and Derivation of Eq.(4.21a-b)

We write the sixteen component Dirac spinor as

$$
\psi=\left[\begin{array}{l}
\psi_{1} \\
\psi_{2} \\
\psi_{3} \\
\psi_{4}
\end{array}\right]
$$

in which the $\psi_{i}$ are four component spinors. All the matrices that operate on this spinor are sixteen by sixteen. In the standard Dirac representation (the subscripts on the identity 1 gives the dimensionality of the uni matrix.)

$$
\begin{aligned}
& \beta_{1}=\left(\begin{array}{cc}
1_{8} & 0 \\
0 & -1_{8}
\end{array}\right), \quad \gamma_{51}=\left(\begin{array}{cc}
0 & 1_{8} \\
1_{8} & 0
\end{array}\right), \quad \beta_{1} \gamma_{51} \equiv \rho_{1}=\left(\begin{array}{cc}
0 & 1_{8} \\
-1_{8} & 0
\end{array}\right) \\
& \beta_{2}=\left(\begin{array}{ll}
\beta & 0 \\
0 & \beta
\end{array}\right), \beta=\left(\begin{array}{cc}
1_{4} & 0 \\
0 & -1_{4}
\end{array}\right) \\
& \gamma_{52}=\left(\begin{array}{cc}
\gamma_{5} & 0 \\
0 & \gamma_{5}
\end{array}\right), \gamma_{5}=\left(\begin{array}{cc}
0 & 1_{4} \\
1_{4} & 0
\end{array}\right) \\
& \beta_{2} \gamma_{52} \equiv \rho_{2}=\left(\begin{array}{ll}
\rho & 0 \\
0 & \rho
\end{array}\right), \rho=\left(\begin{array}{cc}
0 & 1_{4} \\
-1_{4} & 0
\end{array}\right) \\
& \beta_{1} \gamma_{51} \gamma_{52}=\left(\begin{array}{cc}
0 & \gamma_{5} \\
-\gamma_{5} & 0
\end{array}\right) \\
& \beta_{2} \gamma_{52} \gamma_{51}=\left(\begin{array}{ll}
0 & \rho \\
\rho & 0
\end{array}\right) \text {. }
\end{aligned}
$$

Thus Eqs.(2.18a-b) become the 8 coupled equations:

$$
\left(-G \sigma_{1} \cdot \mathcal{P}_{2}\right) \psi_{1}+\left(E_{1}+M_{1}\right) \psi_{3}+\frac{i G}{2} \sigma_{2} \cdot(\partial J-\partial L) \psi_{4}=0
$$




$$
\begin{aligned}
& \left(-G \sigma_{1} \cdot \mathcal{P}_{2}\right) \psi_{2}+\left(E_{1}+M_{1}\right) \psi_{4}+\frac{i G}{2} \sigma_{2} \cdot(\partial J+\partial L) \psi_{3}=0 \\
& \left(G \sigma_{1} \cdot \mathcal{P}_{2}\right) \psi_{3}-\left(E_{1}-M_{1}\right) \psi_{1}-\frac{i G}{2} \sigma_{2} \cdot(\partial J+\partial L) \psi_{2}=0 \\
& \left(G \sigma_{1} \cdot \mathcal{P}_{2}\right) \psi_{4}-\left(E_{1}-M_{1}\right) \psi_{2}-\frac{i G}{2} \sigma_{2} \cdot(\partial J-\partial L) \psi_{1}=0 \\
& \left(G \sigma_{2} \cdot \mathcal{P}_{1}\right) \psi_{1}+\left(E_{2}+M_{2}\right) \psi_{2}-\frac{i G}{2} \sigma_{1} \cdot(\partial J-\partial L) \psi_{4}=0 \\
& \left(-G \sigma_{2} \cdot \mathcal{P}_{1}\right) \psi_{2}-\left(E_{2}-M_{2}\right) \psi_{1}+\frac{i G}{2} \sigma_{1} \cdot(\partial J+\partial L) \psi_{3}=0 \\
& \left(G \sigma_{2} \cdot \mathcal{P}_{1}\right) \psi_{3}+\left(E_{2}+M_{2}\right) \psi_{4}-\frac{i G}{2} \sigma_{1} \cdot(\partial J+\partial L) \psi_{2}=0 \\
& \left(-G \sigma_{2} \cdot \mathcal{P}_{1}\right) \psi_{4}-\left(E_{2}-M_{2}\right) \psi_{3}+\frac{i G}{2} \sigma_{1} \cdot(\partial J-\partial L) \psi_{1}=0
\end{aligned}
$$

in which

$$
\mathcal{P}_{i} \equiv p-\frac{i}{2} \sigma_{i} \cdot \partial \ln G \sigma_{i}
$$

Without the effect of recoil, the terms at the end disappear, $G$ is replaced by 1 (so that $\mathcal{P} \rightarrow p$ ) so that these equations take the same form as the ordinary one-body Dirac equations. Accordingly we perform a standard reduction usually applied to the one-body Dirac equation. We rewrite Eq.(B.8) as

$$
\psi_{3}=\frac{1}{\left(E_{1}+M_{1}\right)}\left[\left(G \sigma_{1} \cdot \mathcal{P}_{2}\right) \psi_{1}-\frac{i G}{2} \sigma_{2} \cdot(\partial J-\partial L) \psi_{4}\right]
$$

We rewrite Eq.(B.12) as

$$
\psi_{2}=\frac{1}{\left(E_{2}+M_{2}\right)}\left[\left(-G \sigma_{2} \cdot \mathcal{P}_{1}\right) \psi_{1}+\frac{i G}{2} \sigma_{1} \cdot(\partial J-\partial L) \psi_{4}\right]
$$

Substitution of Eqs.(B.17,B.18) into Eq.(B.10) then yields

$$
\left(G \sigma_{1} \cdot \mathcal{P}_{2}\right) \frac{1}{\left(E_{1}+M_{1}\right)}\left[\left(G \sigma_{1} \cdot \mathcal{P}_{2}\right) \psi_{1}-\frac{i G}{2} \sigma_{2} \cdot(\partial J-\partial L) \psi_{4}\right]
$$




$$
\left.-\left(E_{1}-M_{1}\right) \psi_{1}-\frac{i G}{2} \sigma_{2} \cdot(\partial J+\partial L) \frac{1}{\left(E_{2}+M_{2}\right)}\left[-G \sigma_{2} \cdot \mathcal{P}_{1}\right) \psi_{1}+\frac{i G}{2} \sigma_{1} \cdot(\partial J-\partial L) \psi_{4}\right]=0
$$

We combine terms to obtain

$$
\begin{gathered}
\left\{\left(G \sigma_{1} \cdot \mathcal{P}_{2}\right) \frac{1}{\left(E_{1}+M_{1}\right)}\left(G \sigma_{1} \cdot \mathcal{P}_{2}\right)-\left(E_{1}-M_{1}\right)\right. \\
\left.-\frac{i G}{2} \sigma_{2} \cdot(\partial J+\partial L) \frac{1}{\left(E_{2}+M_{2}\right)}\left(-G \sigma_{2} \cdot \mathcal{P}_{1}\right)\right\} \psi_{1} \\
+\left\{-\left(G \sigma_{1} \cdot \mathcal{P}_{2}\right) \frac{1}{\left(E_{1}+M_{1}\right)} \frac{i G}{2} \sigma_{2} \cdot(\partial J-\partial L)\right. \\
\left.-\frac{i G}{2} \sigma_{2} \cdot(\partial J+\partial L) \frac{1}{\left(E_{2}+M_{2}\right)} \frac{i G}{2} \sigma_{1} \cdot(\partial J-\partial L)\right\} \psi_{4}=0
\end{gathered}
$$

Since $B A B^{-1}=A+[B, A] B^{-1}$, multiplication of Eq.(B.20) by $\left(E_{1}+M_{1}\right)$ yields

$$
\begin{gathered}
\left\{\left(G \sigma_{1} \cdot \mathcal{P}_{2}\right)^{2}+i G^{2} \partial \ln \left(E_{1}+M_{1}\right) \cdot \sigma_{1} \sigma_{1} \cdot \mathcal{P}_{2}\right. \\
\left.-\left(E_{1}^{2}-M_{1}^{2}\right)+\left(E_{1}+M_{1}\right) \frac{i G}{2} \sigma_{2} \cdot \partial(J+L) \frac{1}{E_{2}+M_{2}} G \sigma_{2} \cdot \mathcal{P}_{1}\right\} \psi_{1} \\
+\left\{-\frac{i G}{2} \sigma_{1} \cdot \mathcal{P}_{2} G \sigma_{2} \cdot \partial(J-L)+\frac{1}{2} G^{2} \partial \ln \left(E_{1}+M_{1}\right) \cdot \sigma_{1} \sigma_{2} \cdot \partial(J-L)\right. \\
\left.-\frac{i G}{2} \sigma_{2} \cdot(\partial J+\partial L) \frac{\left(E_{1}+M_{1}\right)}{\left(E_{2}+M_{2}\right)} \frac{i G}{2} \sigma_{1} \cdot(\partial J-\partial L)\right\} \psi_{4}=0 .
\end{gathered}
$$

We simplify this equation by finding another equation that relates $\psi_{1}$ and $\psi_{4}$ but which involves only first order appearances of $\sigma_{1} \cdot \mathcal{P}_{2}$ and $\sigma_{2} \cdot \mathcal{P}_{1}$. We first multiply Eq.(B.11) by $\frac{i G}{2} \partial(J-L) \cdot \sigma_{2}$ to ot tain

$$
\begin{gathered}
\frac{i G}{2} \partial(J-L) \cdot \sigma_{2}\left(G \sigma_{1} \cdot \mathcal{P}_{2}\right) \psi_{4}-\frac{i G}{2} \partial(J-L) \cdot \sigma_{2}\left(E_{1}-M_{1}\right) \psi_{2} \\
+\frac{G^{2}}{4} \sigma_{2} \cdot(\partial J-\partial L) \sigma_{2} \cdot(\partial J-\partial L) \psi_{1}=0
\end{gathered}
$$


Note that $\frac{i G}{2} \partial(J-L) \cdot \sigma_{2}$ has no nontrivial eigeuvector with a zero eigenvalue so that when multiplying by it we do not introduce any extra solutions to our Dirac equations. If we multiply Eq.(B.12) by

$$
\frac{i G}{2} \partial(J-L) \cdot \sigma_{2} \frac{\left(E_{1}-M_{1}\right)}{\left(E_{2}+M_{2}\right)}
$$

(which also has no nontrivial eigenvector with a zero eigenvalue) we obtain

$$
\begin{gathered}
\frac{i G}{2} \partial(J-L) \cdot \sigma_{2} \frac{\left(E_{1}-M_{1}\right)}{\left(E_{2}+M_{2}\right)}\left(G \sigma_{2} \cdot \mathcal{P}_{1}\right) \psi_{1}-\frac{i G}{2} \partial(J-L) \cdot \sigma_{2}\left(E_{1}-M_{1}\right) \psi_{2} \\
-\frac{i G}{2} \partial(J-L) \cdot \sigma_{2} \frac{\left(E_{1}-M_{1}\right)}{\left(E_{2}+M_{2}\right)} \frac{i G}{2} \sigma_{1} \cdot(\partial J-\partial L) \psi_{4}=0 .
\end{gathered}
$$

Addition of this to Eq.(B.22) then yields

$$
\begin{gathered}
\left\{\frac{i G}{2} \partial(J-L) \cdot \sigma_{2} \frac{\left(E_{1}-M_{1}\right)}{\left(E_{2}+M_{2}\right)}\left(G \sigma_{2} \cdot \mathcal{P}_{1}\right)+\frac{G^{2}}{4}(\partial J-\partial L)^{2}\right\} \psi_{1} \\
+\left\{\frac{i G}{2} \partial(J-L) \cdot \sigma_{2}\left(G \sigma_{1} \cdot \mathcal{P}_{2}\right)-\frac{i G}{2} \partial(J-L) \cdot \sigma_{2} \frac{\left(E_{1}-M_{1}\right)}{\left(E_{2}+M_{2}\right)} \frac{i G}{2} \sigma_{1} \cdot(\partial J-\partial L)\right\} \psi_{4}=0 .
\end{gathered}
$$

Now, note that

$$
\left(E_{1}+M_{1}\right) \partial(J+L)+\left(E_{1}-M_{1}\right) \partial(J-L)=2\left(E_{1} \partial J+M_{1} \partial L\right)=2 \partial\left(E_{2}+M_{2}\right)
$$

We then add Eq.(B.21) to Eq.(B.24) and use the previous equation to obtain

$$
\begin{gathered}
\left\{\left(G \sigma_{1} \cdot \mathcal{P}_{2}\right)^{2}-\left(E_{1}^{2}-M_{1}^{2}\right)+\frac{G^{2}}{4}(\partial J-\partial L)^{2}\right. \\
\left.+i G^{2} \partial \ln \left(E_{1}+M_{1}\right) \cdot \sigma_{1} \sigma_{1} \cdot \mathcal{P}_{2}+i G^{2} \partial \ln \left(E_{2}+M_{2}\right) \cdot \sigma_{2} \sigma_{2} \cdot \mathcal{P}_{1}\right\} \psi_{1} \\
+\left\{+\frac{1}{2} G^{2} \partial \ln \left(E_{1}+M_{1}\right) \cdot \sigma_{1} \sigma_{2} \cdot \partial(J-L)+\frac{1}{2} G^{2} \partial \ln \left(E_{2}+M_{2}\right) \cdot \sigma_{2} \sigma_{1} \cdot \partial(J-L)\right.
\end{gathered}
$$




$$
\left.-\frac{i G}{2}\left[\sigma_{1} \cdot \mathcal{P}_{2}, G \sigma_{2} \cdot \partial(J-L)\right]\right\} \psi_{4}=0
$$

In the Dirac representation of the Dirac gamma matrices, Eq.(4.12) has an upper-upper component that is equivalent to Eq.(B.26a). In a similar way one could obtain the upper-lower, lower-upper, and lower-lower components of Eq.(4.12) without squaring and substituting. Thus the result Eq.(4.12) obtained by squaring and substituting (as done in Eqs.(4.1011 ) in section IV) is equivalent to that obtained here.

Eq.(B.26a) is coupled to the lower-lower component of Eq.(4.12) below

$$
\begin{gathered}
\left\{\left(G \sigma_{1} \cdot \mathcal{P}_{2}\right)^{2}-\left(E_{1}^{2}-M_{1}^{2}\right)+\frac{G^{2}}{4}(\partial J-\partial L)^{2}\right. \\
\left.+i G^{2} \partial \ln \left(E_{1}-M_{1}\right) \cdot \sigma_{1} \sigma_{1} \cdot \mathcal{P}_{2}+i G^{2} \partial \ln \left(E_{2}-M_{2}\right) \cdot \sigma_{2} \sigma_{2} \cdot \mathcal{P}_{1}\right\} \psi_{4} \\
+\left\{+\frac{1}{2} G^{2} \partial \ln \left(E_{1}-M_{1}\right) \cdot \sigma_{1} \sigma_{2} \cdot \partial(J-L)+\frac{1}{2} G^{2} \partial \ln \left(E_{2}-M_{2}\right) \cdot \sigma_{2} \sigma_{1} \cdot \partial(J-L)\right. \\
\left.-\frac{i G}{2}\left[\sigma_{1} \cdot \mathcal{P}_{2}, G \sigma_{2} \cdot \partial(J-L)\right]\right\} \psi_{1}=0 .
\end{gathered}
$$

We now perform the indicated Pauli matrix multiplication in Eq.(B.26a) and Eq.(B.26b) to obtain

$$
\begin{gathered}
\left(G \sigma_{1} \cdot \mathcal{P}_{2}\right)^{2}=G^{2} p^{2}-2 i G \partial G \cdot p+G \partial G \times p \cdot\left(\sigma_{1}+\sigma_{2}\right)^{-} \\
-\frac{1}{2} G \partial^{2} G\left(1-\sigma_{1} \cdot \sigma_{2}\right)-\frac{1}{2} G \sigma_{1} \cdot \partial \sigma_{2} \cdot \partial G \\
\quad+\frac{1}{4}(\partial G)^{2}-\frac{1}{2} \partial G \cdot \sigma_{1} \partial G \cdot \sigma_{2} .
\end{gathered}
$$

Then Eq.(B.12), Eq.(B.13), and $\epsilon_{1}^{2}-m_{1}^{2}=b^{2}(w)$ lead to

$$
E_{1}^{2}-M_{1}^{2}=G^{2}\left(-b^{2}(w)+2 m_{w} S+S^{2}+2 \epsilon_{w} \mathcal{A}-\mathcal{A}^{2}+2 \epsilon_{w} \mathcal{V}-\mathcal{V}^{2}\right)
$$


Since $\mathcal{P}_{i}$ is as defined in Eq.(B.16) and since

$$
\begin{gathered}
i \sigma \cdot \partial() \sigma \cdot p=i \partial() \cdot p-\partial() \times p \cdot \sigma \\
A \cdot \sigma_{1} \sigma_{2} \cdot B \sigma_{1} \cdot \sigma_{2}=A \cdot B\left(1-\sigma_{1} \cdot \sigma_{2}\right)+A \cdot \sigma_{1} B \cdot \sigma_{2} \\
+i A \times B \cdot\left(\sigma_{2}-\sigma_{1}\right)
\end{gathered}
$$

we find

$$
\begin{aligned}
& +i G^{2} \partial \ln \left(E_{1}+M_{1}\right) \cdot \sigma_{1} \sigma_{1} \cdot \mathcal{P}_{2}=i G^{2} \partial \ln \left(E_{1}+M_{1}\right) \cdot p-G^{2} \partial \ln \left(E_{1}+M_{1}\right) \times p \cdot \sigma_{1} \\
& +\frac{1}{2} \partial \ln \left(E_{1}+M_{1}\right) \cdot \partial G\left(1-\sigma_{1} \cdot \sigma_{2}\right)+\frac{1}{2} \partial \ln \left(E_{2}+M_{2}\right) \cdot \sigma_{2} \partial G \cdot \sigma_{1} \cdot(B .33)
\end{aligned}
$$

(We perform a similar reduction of a corresponding term in (B.26b)).) The commutator term in Eq.(B.26a) becomes

$$
\begin{gathered}
-\frac{i G}{2}\left[\sigma_{1} \cdot \mathcal{P}_{2}, G \sigma_{2} \cdot \partial(J-L)\right]=-\frac{1}{2} G \sigma_{1} \cdot \partial G \sigma_{2} \cdot \partial(J-L) \\
-G^{2} \frac{1}{2} \sigma_{1} \sigma_{2}: \partial \partial(J-L)-G \frac{1}{2} \sigma_{1} \cdot \partial(J-L) \sigma_{2} \cdot \partial G+\frac{1}{2} G \sigma_{1} \cdot \sigma_{2} \partial G \cdot \partial(J-L) .
\end{gathered}
$$

We perform a similar manipulation on the commutator in Eq.(B.26b). We then combine the terms, divide by $G^{2}$, and use

$$
\begin{gathered}
\frac{1}{G} \partial \partial G=\partial \partial \ln G+(\partial \ln G)^{2} \\
\partial()=\hat{r}()^{\prime} \\
\frac{1}{G} \sigma_{1} \cdot \partial \sigma_{2} \cdot \partial G=\sigma_{1} \cdot \sigma_{2} \frac{\ln ^{\prime} G}{r}+\sigma_{1} \cdot \hat{r} \sigma_{2} \cdot \hat{r}\left(\ln n^{\prime \prime} G-\frac{\ln ^{\prime} G}{r}\right)+\sigma_{1} \cdot \hat{r} \sigma_{2} \cdot \hat{r}\left(\ln ^{\prime} G\right)^{2} \\
\hat{r} \cdot \sigma_{1} \hat{r} \cdot \sigma_{2}=\frac{1}{3}\left(S_{T}+\sigma_{1} \cdot \sigma_{2}\right) .
\end{gathered}
$$

In this way we obtain Eq.(4.21a-b) in section IV from Eq.(B.26a-b) without squaring. 
C- Derivation of the Perturbative Spectrum from the Constraint Equations

We use the general wave function

$$
\psi_{n j}=c_{1} \psi_{n j 0 j}+c_{2} \psi_{n j 1 j}, j \geq 1
$$

to diagonalize the perturbation for the $j=l \geq 1$ states or the general wave function

$$
\psi_{n j}=c_{3} \psi_{n j-11 j}+c_{4} \psi_{n j+11 j}, j \geq 1
$$

for the $j=l \pm 1$ states. The corresponding identifications for the $j=0$ states are

$$
\psi_{n 0}=\psi_{n 000}
$$

or

$$
\psi_{n 0}=\psi_{n 110}
$$

For these wave functions, the relevent matrix elements become

$$
\begin{gathered}
<n l s j m\left|\frac{1}{x^{2}}\right| n l s j m>=\frac{2}{n^{3}(2 l+1)} \\
<n l s j m\left|\frac{1}{x^{2}} \frac{d}{d x}\right| n l s j m>=-\delta_{l 0} \frac{2}{n^{3}} \\
<n l s j m\left|\delta^{3}(\vec{x})\right| n l s j m>=\delta_{l 0} \frac{1}{\pi n^{3}} \\
<n l s j m\left|\frac{1}{x^{3}}\right| n l s j m>\left(1-\delta_{l 0}\right)=\frac{2}{n^{3}(2 l+1) l(l+1)}\left(1-\delta_{l 0}\right) \\
<n l s j m\left|\vec{L} \cdot\left(\vec{\sigma}_{1}+\vec{\sigma}_{2}\right)\right| n l s j m>=\left(1-\delta_{l 0}\right)\left(1-\delta_{s 0}\right)(j(j+1)-l(l+1)-s(s+1))
\end{gathered}
$$




$$
\begin{gathered}
<n l s j m\left|S_{T}\right| n l s j m>=\left(-\frac{2 l}{2 l+3} \delta_{j l+1}+2 \delta_{j l}-\frac{2(l+1)}{(2 l-1)} \delta_{j l-1}\right)\left(1-\delta_{s 0}\right) \\
\equiv \kappa_{l j}\left(1-\delta_{l 0}\right)\left(1-\delta_{s 0}\right) \\
<n j-1 s j m\left|S_{T}\right| n j+1 s j m>=\frac{6 \sqrt{(j(j+1)}}{2 j+1}\left(1-\delta_{s 0}\right)=<n j+1 s j m\left|S_{T}\right| n j-1 s j m>
\end{gathered}
$$
and

$$
<n l s j m\left|\vec{L} \cdot\left(\vec{\sigma}_{1}-\vec{\sigma}_{2}\right)\right| n l s^{\prime} j m>=\delta_{l j}\left(1-\delta_{l 0}\right)\left(1-\delta_{s s^{\prime}}\right) 2 \sqrt{(l(l+1)}
$$

For the $j=0$ states Eq.(5.9),Eq.(5.12), and Eq.(5.15) yield the dimensionless eigenvalue (defined in Sec. V)

$$
-\lambda=-\frac{1}{n^{2}}+\frac{\alpha^{4}}{n^{3}} \eta
$$

in which

$$
\begin{gathered}
\eta=-\frac{2}{2 l+1}+\delta_{l 0}+\frac{(-l(l+1)-2)\left(\frac{\mu}{M}+\frac{1}{2}\right)+\kappa_{l j} \frac{\mu}{M}}{(2 l+1) l(l+1)}\left(1-\delta_{l 0}\right)\left(1-\delta_{s 0}\right) \\
+\frac{8 \mu}{3 M} \delta_{l 0}\left[s(s+1)-\frac{3}{2}\right]
\end{gathered}
$$

for electromagnetic interactions,

$$
\begin{gathered}
\eta=\frac{2}{2 l+1}-\delta_{l 0}\left(1-\frac{2 \mu}{M}\right) \\
+\frac{(-l(l+1)-2)\left(\frac{\mu}{M}-\frac{1}{2}\right)}{(2 l+1) l(l+1)}\left(1-\delta_{l 0}\right)\left(1-\delta_{s 0}\right)
\end{gathered}
$$

for scalar interactions and

$$
\eta=-\frac{2}{2 l+1}+\delta_{l 0}\left(1-\frac{2 \mu}{M}\right)
$$




$$
+\frac{(-l(l+1)-2)\left(\frac{\mu}{M}-\frac{1}{2}\right)}{(2 l+1) l(l+1)}\left(1-\delta_{l 0}\right)\left(1-\delta_{s 0}\right)
$$

for time-like vector interactions. We then use the definition of $\lambda$ given in Sec. V for electromagnetic and time-like interactions to obtain

$$
\frac{\epsilon_{w}}{m_{w}}=\left[1+\frac{\alpha^{2}}{n^{2}}-\frac{\alpha^{4} \eta_{ \pm}}{n^{3}}\right]^{-\frac{1}{2}}
$$

which leads to the binding energies

$$
\epsilon_{B}=w-M=-\frac{\mu \alpha^{2}}{2 n^{2}}+\frac{\mu \alpha^{4}}{2 n^{3}} \eta+\frac{\mu \alpha^{4}}{8 n^{4}}\left(3-\frac{\mu}{M}\right)
$$

For the case of scalar dynamics the definition of $\lambda$ (in Sec. V) is different and yields

$$
\frac{\epsilon_{w}}{m_{w}}=\left[1-\frac{\alpha^{2}}{n^{2}}+\frac{\alpha^{4} \eta}{n^{3}}\right]^{\frac{1}{2}}
$$

which results in the binding energies

$$
\epsilon_{B}=w-M=-\frac{\mu \alpha^{2}}{2 n^{2}}+\frac{\mu \alpha^{4}}{2 n^{3}} \eta+\frac{\mu \alpha^{4}}{8 n^{4}}\left(-1-\frac{\mu}{M}\right) .
$$

For the case $j=l \geq 1$, the expectation values of each of Eqs.(5.9,5.12,5.15) with the wave function Eq.(C.1) lead to

$$
\begin{aligned}
& {\left[-\frac{1}{n^{2}}+\frac{\alpha^{2}}{n^{3}} a\right] c_{1}+\frac{\alpha^{2}}{n^{3}} b c_{2}=-\lambda c_{1}} \\
& {\left[-\frac{1}{n^{2}}+\frac{\alpha^{2}}{n^{3}} c\right] c_{2}+\frac{\alpha^{2}}{n^{3}} b c_{1}=-\lambda c_{2} .}
\end{aligned}
$$

Solution of the determinantal condition for these equations yields two roots corresponding to the mixed spin states:

$$
-\lambda=-\frac{1}{n^{2}}+\frac{\alpha^{2}}{2 n^{3}}\left(a+c \pm \sqrt{(a-c)^{2}+4 b^{2}}\right) \equiv-\frac{1}{n^{2}}+\frac{\alpha^{2}}{n^{3}} \eta_{ \pm} .
$$


We term the upper root the "singlet root" and the lower one the "triplet root", since as $b \rightarrow 0$ Eq.(C.22) degenerate to those roots. For the electromagnetic-like interactions, we find

$$
\begin{gathered}
a=-\frac{2}{2 l+1}, \\
b=\frac{1}{(2 l+1) \sqrt{(l(l+1)}} \frac{m_{2}-m_{1}}{M},
\end{gathered}
$$

while

$$
c=-\frac{2}{2 l+1}-\frac{1}{(2 l+1) l(l+1)} .
$$

For the case of scalar interactions

$$
\begin{gathered}
a=\frac{2}{2 l+1}, \\
b=-\frac{1}{(2 l+1) \sqrt{(l(l+1)}} \frac{m_{2}-m_{1}}{M}
\end{gathered}
$$

while

$$
c=\frac{2}{2 l+1}+\frac{1}{(2 l+1) l(l+1)}\left(1-\frac{2 \mu}{M}\right) .
$$

For the case of time-like vector interactions,

$$
\begin{gathered}
a=-\frac{2}{2 l+1}, \\
b=\frac{1}{(2 l+1) \sqrt{(l(l+1)}}=\frac{m_{2}-m_{1}}{M},
\end{gathered}
$$

while

$$
c=-\frac{2}{2 l+1}-\frac{1}{(2 l+1) l(l+1)}\left(1-\frac{2 \mu}{M}\right) .
$$


Using the definitions of $\lambda$ (in Sec.V) we find the two binding energies for the split spin-mixed states for electromagnetic-like or time-like vector interactions:

$$
\epsilon_{B}=w-M=-\frac{\mu \alpha^{2}}{2 n^{2}}+\frac{\mu \alpha^{4}}{2 n^{3}} \eta_{ \pm}+\frac{\mu \alpha^{4}}{8 n^{4}}\left(3-\frac{\mu}{M}\right) .
$$

Similarly, for scalar interactions

$$
\epsilon_{B}=w-M=-\frac{\mu \alpha^{2}}{2 n^{2}}+\frac{\mu \alpha^{4}}{2 n^{3}} \eta_{ \pm}+\frac{\mu \alpha^{4}}{8 n^{4}}\left(-1-\frac{\mu}{M}\right)
$$

The values of $\eta_{ \pm} \equiv\left(a+c \pm \sqrt{(a-c)^{2}+4 b^{2}}\right)$ then depend on the form of interaction through the relevant $a, b$, and $c$ given above.

Next we consider the $j=l \pm 1 \geq 1$ spectral equations. Taking expectation values with the wave functions Eq.(C.2), we obtain

$$
\begin{aligned}
& {\left[-\frac{1}{n^{2}}+\frac{\alpha^{2}}{n^{3}} a\right] c_{3}+\frac{\alpha^{2}}{n^{3}} b c_{4}=-\lambda c_{3}} \\
& {\left[-\frac{1}{n^{2}}+\frac{\alpha^{2}}{n^{3}} c\right] c_{4}+\frac{\alpha^{2}}{n^{3}} b c_{3}=-\lambda c_{4} .}
\end{aligned}
$$

Solution of the determinantal condition for these equations yields two roots corresponding to the mixed orbital states

$$
-\lambda=-\frac{1}{n^{2}}+\frac{\alpha^{2}}{2 n^{3}}\left(a+c \pm \sqrt{(a-c)^{2}+4 b^{2}}\right) \equiv-\frac{1}{n^{2}}+\frac{\alpha^{2}}{n^{3}} \eta_{ \pm} .
$$

Note that $b=0$ for the $n=1,2$ levels since there are no $l=j+1 \geq 2$ states. $b$ also vanishes for scalar and time-like interactions since these interactions have no tensor terms through order $\alpha^{4}$. So for all of these states

$$
-\lambda=-\frac{1}{n^{2}}+\frac{\alpha^{2}}{2 n^{3}} \eta
$$


For the electromagnetic-like interactions (with $n=1,2$ )

$$
\begin{gathered}
\eta=-\frac{2}{2 l+1}+\delta_{l 0}+\frac{(j(j+1)-l(l+1)-2)\left(\frac{\mu}{M}+\frac{1}{2}\right)+\kappa_{l j} \frac{\mu}{M}}{(2 l+1) l(l+1)}\left(1-\delta_{l 0}\right)\left(1-\delta_{s 0}\right) \\
+\frac{8 \mu}{3 M} \delta_{l 0}\left(s(s+1)-\frac{3}{2}\right) .
\end{gathered}
$$

For scalar interactions (all $n$ )

$$
\begin{gathered}
\eta=\frac{2}{2 l+1}-\delta_{l 0}\left(1-\frac{2 \mu}{M}\right) \\
+\frac{(j(j+1)-l(l+1)-2)\left(\frac{\mu}{M}-\frac{1}{2}\right)}{(2 l+1) l(l+1)}\left(1-\delta_{l 0}\right)\left(1-\delta_{s 0}\right),
\end{gathered}
$$

while for time-like vector interactions (for all $n$ )

$$
\begin{gathered}
\eta=-\frac{2}{2 l+1}+\delta_{l 0}\left(1-\frac{2 \mu}{M}\right) \\
+\frac{(-l(l+1)-2)\left(\frac{\mu}{M}-\frac{1}{2}\right)}{(2 l+1) l(l+1)}\left(1-\delta_{l 0}\right)\left(1-\delta_{s 0}\right) .
\end{gathered}
$$

Just as for the $j=0$ states, for the case of electromagnetic- and time-like interactions the binding energies are given by

$$
\epsilon_{B}=w-M=-\frac{\mu \alpha^{2}}{2 n^{2}}+\frac{\mu \alpha^{4}}{2 n^{3}} \eta+\frac{\mu \alpha^{4}}{8 n^{4}}\left(3-\frac{\mu}{M}\right)
$$

whereas for scalar interactions the binding energies are

$$
\epsilon_{B}=w-M=-\frac{\mu \alpha^{2}}{2 n^{2}}+\frac{\mu \alpha^{4}}{2 n^{3}} \eta+\frac{\mu \alpha^{4}}{8 n^{4}}\left(-1-\frac{\mu}{M}\right) .
$$

In all cases, $j=l \pm 1 \geq 1$.

For the electromagnetic interactions with $n \geq 3$ the (split) energy spectrum turns out to be

$$
\epsilon_{B}=w-M=-\frac{\mu \alpha^{2}}{2 n^{2}}+\frac{\mu \alpha^{4}}{2 n^{3}} \eta_{ \pm}+\frac{\mu \alpha^{4}}{8 n^{4}}\left(3-\frac{\mu}{M}\right)
$$


in which

$$
\eta_{ \pm}=\left(a+c \pm \sqrt{(a-c)^{2}+4 b^{2}}\right)
$$

where

$$
\begin{gathered}
a=-\frac{2}{2 l+1}+\delta_{l 0}+\frac{2 l\left(\frac{\mu}{M}+\frac{1}{2}\right)+\kappa_{l l+1} \frac{\mu}{M}}{(2 l+1) l(l+1)}\left(1-\delta_{l 0}\right)\left(1-\delta_{s 0}\right) \\
+\frac{8 \mu}{3 M} \delta_{l 0}\left[s(s+1)-\frac{3}{2}\right] \\
b=\frac{6 \sqrt{(j(j+1)}}{2 j+1}\left(1-\delta_{s 0}\right) \zeta_{n j} \\
c=-\frac{2}{2 l+1}+\delta_{l 0}+\frac{(-2 l-2)\left(\frac{\mu}{M}+\frac{1}{2}\right)+\kappa_{l l-1} \frac{\mu}{M}}{(2 l+1) l(l+1)}\left(1-\delta_{l 0}\right)\left(1-\delta_{s 0}\right) .
\end{gathered}
$$

In Eq.(C.39), $\zeta_{n j}$ is the overlap radial integral $\left\langle n j+1\left|\frac{1}{x^{3}}\right| n j-1\right\rangle$ between the $n, j=l \pm 1$ states. Surprisingly, this integral vanishes. We omit the details here other than to point out that use of the contour integral representation of the Laguerre polynomials leads to a simple demonstration that the overlap integral vanishes. As a result the spectral results for $n \geq 3$ have the same form as those for $n=1,2$ for the electromagnetic interaction. We summarize the results in the text. 
D Derivation of the Decoupled Schrödinger-like Forms of Eqs.(4.21a-b) for the $j=l$ and ${ }^{3} P_{0}$ States

In Refs.(4-5), we attempted to decouple the upper-upper from the lower-lower components in (4.21a-b) by obtaining a purely algebraic equation relating $\psi_{4}$ to $\psi_{1}$ and substituting this into the equivalent of Eq.(4.21a). In the attempt, we obtained a result which was true for the $j=l$ states but not true for the $j=l \pm 1$ states. Consequently, in order to give a uniform treatment to all the bound-state solutions of Sec. VI, we used the coupled equations (4.21a-b) directly. Here we present an alternative derivation of the decoupling for the $j=l$ states and encounter an error made in Refs.(4-5) in which we attempted to extend this result to obtain a similar algebraic equation for the $j=l \pm 1$ states. We shall also obtain a decoupled form of Eqs.(4.21a-b) for the ${ }^{3} P_{0}$ state.

As in Refs.(4-5) we begin by multiplying Eq.(B-8) by $\frac{i G}{2} \partial(J-L) \cdot \sigma_{2}$ to obtain

$$
\begin{gathered}
-\frac{i G}{2} \partial(J-L) \cdot \sigma_{2}\left(G \sigma_{1} \cdot \mathcal{P}_{2}\right) \psi_{1}+\frac{i G}{2} \partial(J-L) \cdot \sigma_{2}\left(E_{1}+M_{1}\right) \psi_{3}-\frac{G^{2}}{4}(\partial J-\partial L)^{2} \psi_{4} \\
=0 .
\end{gathered}
$$

If we also multiply Eq.(B-15) by

$$
\frac{i G}{2} \partial(J-L) \cdot \sigma_{2} \frac{\left(E_{1}+M_{1}\right)}{\left(E_{2}-M_{2}\right)}
$$

we obtain

$$
\begin{gathered}
-\frac{i G}{2} \partial(J-L) \cdot \sigma_{2} \frac{\left(E_{1}+M_{1}\right)}{\left(E_{2}-M_{2}\right)}\left(G \sigma_{2} \cdot \mathcal{P}_{1}\right) \psi_{4}-\frac{i G}{2} \partial(J-L) \cdot \sigma_{2}\left(E_{1}+M_{1}\right) \psi_{3} \\
\quad+\frac{i G}{2} \partial(J-L) \cdot \sigma_{2} \frac{\left(E_{1}+M_{1}\right)}{\left(E_{2}-M_{2}\right)} \frac{i G}{2} \sigma_{2} \cdot(\partial J-\partial L) \psi_{1}=0 .
\end{gathered}
$$


We add this to Eq.(D.1) to obtain

$$
\begin{gathered}
\left\{-\frac{i G}{2} \partial(J-L) \cdot \sigma_{2} \frac{\left(E_{1}+M_{1}\right)}{\left(E_{2}-M_{2}\right)}\left(G \sigma_{2} \cdot \mathcal{P}_{1}\right)-\frac{G^{2}}{4}(\partial J-\partial L)^{2}\right\} \psi_{4} \\
+\left\{-\frac{i G}{2} \partial(J-L) \cdot \sigma_{2}\left(G \sigma_{1} \cdot \mathcal{P}_{2}\right)+\frac{i G}{2} \partial(J-L) \cdot \sigma_{2} \frac{\left(E_{1}+M_{1}\right)}{\left(E_{2}-M_{2}\right)} \frac{i G}{2} \sigma_{1} \cdot(\partial J-\partial L)\right\} \psi_{1} \\
=0
\end{gathered}
$$

We then use the expression for $\mathcal{P}_{1}$ (Eq.(4.8)) and the identity

$$
\sigma_{2} \cdot \hat{r} \sigma_{1} \cdot \hat{r} \sigma_{1} \cdot \sigma_{2}=1-\sigma_{1} \cdot \sigma_{2}+\sigma_{1} \cdot \hat{r} \sigma_{2} \cdot \hat{r}
$$

to simplify Eq.(D.4) to

$$
\begin{gathered}
\left\{\frac{G^{2}}{2}(J-L)^{\prime}\left[i \hat{r} \cdot p-\frac{L \cdot \sigma_{1}}{r}\right]\right. \\
\left.+\frac{G^{2}}{4}(J-L)^{\prime} \mathcal{G}^{\prime}\left(1-\sigma_{1} \cdot \sigma_{2}+\sigma_{1} \cdot \hat{r} \sigma_{2} \cdot \hat{r}\right)+\frac{\left(E_{2}-M_{2}\right)}{\left(E_{1}+M_{1}\right)} \frac{G^{2}}{4}(\partial J-\partial L)^{2}\right\} \psi_{4} \\
+\left\{\frac{\left(E_{2}-M_{2}\right)}{\left(E_{1}+M_{1}\right)} \frac{i G^{2}}{2}(J-L)^{\prime} \hat{r} \cdot \sigma_{1}\left(\sigma_{2} \cdot p\right)+\frac{\left(E_{2}-M_{2}\right)}{\left(E_{1}+M_{1}\right)} \frac{G^{2}}{4}(J-L)^{\prime} \sigma_{1} \cdot \sigma_{2} \mathcal{G}^{\prime}\right. \\
\left.+\frac{G^{2}}{4}\left((J-L)^{\prime}\right)^{2} \sigma_{1} \cdot \hat{r} \sigma_{2} \cdot \hat{r}\right\} \psi_{1}=0
\end{gathered}
$$

in which $\mathcal{G}=\ln G$. When the particle lables are interchanged we obtain the counterpart equation

$$
\begin{gathered}
\left\{\frac{G^{2}}{2}(J-L)^{\prime}\left[i \hat{r} \cdot p-\frac{L \cdot \sigma_{2}}{r}\right]\right. \\
\left.+\frac{G^{2}}{4}(J-L) \mathcal{G}^{\prime}\left(1-\sigma_{1} \cdot \sigma_{2}+\sigma_{1} \cdot \hat{r} \sigma_{2} \cdot \hat{r}\right)+\frac{\left(E_{1}-M_{1}\right)}{\left(E_{2}+M_{2}\right)} \frac{G^{2}}{4}(\partial J-\partial L)^{2}\right\} \psi_{4} \\
+\left\{\frac{\left(E_{1}-M_{1}\right)}{\left(E_{2}+M_{2}\right)} \frac{i G^{2}}{2}(J-L)^{\prime} \hat{r} \cdot \sigma_{2}\left(\sigma_{1} \cdot p\right)+\frac{\left(E_{1}-M_{1}\right)}{\left(E_{2}+M_{2}\right)} \frac{G^{2}}{4}(J-L)^{\prime} \sigma_{1} \cdot \sigma_{2} \mathcal{G}^{\prime}\right.
\end{gathered}
$$




$$
\left.+\frac{G^{2}}{4}\left((J-L)^{\prime}\right)^{2} \sigma_{1} \cdot \hat{r} \sigma_{2} \cdot \hat{r}\right\} \psi_{1}=0
$$

We difference Eq.(D.6) and Eq.(D.7) to obtain

$$
\begin{gathered}
\frac{G^{2}}{2}(J-L)^{\prime} \frac{L \cdot\left(\sigma_{1}-\sigma_{2}\right)}{r} \psi_{4} \\
+\frac{B^{2}}{\left(E_{1}+M_{1}\right)\left(E_{2}+M_{2}\right)} \frac{i G^{2}}{2}(J-L)^{\prime}\left(\hat{r} \cdot \sigma_{2}\left(\sigma_{1} \cdot p\right)-\hat{r} \cdot \sigma_{1}\left(\sigma_{2} \cdot p\right)\right) \psi_{1} \\
=0
\end{gathered}
$$

in which

$$
E_{1}^{2}-M_{1}^{2}=E_{2}^{2}-M_{2}^{2} \equiv B^{2}
$$

For the $j=l$ states we extract an algebraic relation between $\psi_{4}$ and $\psi_{1}$ from (D.8) by using Dirac's trick of multiplying by $\sigma_{1} \cdot \hat{r} \sigma_{2} \cdot \hat{r}$. This converts (D.8) into something more manageable, namely

$$
\begin{gathered}
\sigma_{1} \cdot \hat{r} \sigma_{2} \cdot \hat{r} \frac{G^{2}}{2}(J-L)^{\prime} \frac{L \cdot\left(\sigma_{1}-\sigma_{2}\right)}{r} \psi_{4} \\
-\frac{B^{2}}{\left(E_{1}+M_{1}\right)\left(E_{2}+M_{2}\right)} \frac{i G^{2}}{2}(J-L)^{\prime} \frac{L \cdot\left(\sigma_{1}-\sigma_{2}\right)}{r} \psi_{1}=0 .
\end{gathered}
$$

In order to turn these equations and the ones below into radial equations we need the following angular momentum identities

$$
\begin{gathered}
<r l s j m\left|p^{2}=\left(-\frac{1}{r} \frac{d^{2}}{d r^{2}} r+\frac{l(l+1)}{r^{2}}\right)<r l s j m\right|, \\
<r l s j m\left|i \hat{r} \cdot p=\frac{d}{d r}<r l s j m\right|, \\
<r l 0 j m \mid L \cdot\left(\sigma_{1}+\sigma_{2}\right)=0, \\
\left.<r l 1 j m \mid L \cdot\left(\sigma_{1}+\sigma_{2}\right)=<r l 1 j m \|-2-l(l+1)+j(j+1)\right],
\end{gathered}
$$




$$
\begin{gathered}
<r l 0 j m\left|L \cdot\left(\sigma_{1}-\sigma_{2}\right)=<r l 1 j m\right|\left[\left[1-(j-l)^{2}\right][(j+l)(j+l+2)]\right]^{\frac{1}{2}},(D .15) \\
<r l 1 j m\left|L \cdot\left(\sigma_{1}-\sigma_{2}\right)=<r l 0 j m\right|\left[\left[1-(j-l)^{2}\right][(j+l)(j+l+2)]\right]^{\frac{1}{2}},(D .16) \\
<r l 0 j=l m\left|L \cdot\left(\sigma_{1}-\sigma_{2}\right)=<l 1 j=l m\right| 2[l(l+1)]^{\frac{1}{2}}, \\
<r l 1 j=l m\left|L \cdot\left(\sigma_{1}-\sigma_{2}\right)=<r l 0 j=l m\right| 2[l(l+1)]^{\frac{1}{2}}, \\
<r l 1 j=l \pm 1 m \mid L \cdot\left(\sigma_{1}-\sigma_{2}\right)=0, \\
<r l 0 j m \mid S_{T}=0, \\
<j 1 j m\left|S_{T}=2<j j 1 m\right|, \\
<j+11 j m \mid S_{T}=\left(-\frac{(2 j+4)}{2 j+1}<j+11 j m\left|+6 \frac{\sqrt{(j(j+1)}}{2 j+1)}<j-11 j m\right|,\right. \\
<j-11 j m \mid S_{T}=\left(-\frac{(2 j-2)}{2 j+1}<j-11 j m\left|+6 \frac{\sqrt{(j(j+1)}}{2 j+1)}<j+11 j m\right|,\right. \\
<j+11 j m \mid \sigma_{1} \cdot \hat{r} \sigma_{2} \cdot \hat{r}=\left(-\frac{1}{2 j+1}<j+11 j m\left|+2 \frac{\sqrt{(j(j+1)}}{2 j+1)}<j-11 j m\right|,\right.
\end{gathered}
$$

and

$<j-11 j m \mid \sigma_{1} \cdot \hat{r} \sigma_{2} \cdot \hat{r}=\left(\frac{1}{2 j+1}<j-11 j m\left|+2 \frac{\sqrt{(j(j+1)}}{2 j+1)}<j+11 j m\right|\right.$.

Thus the singlet component of Eq.(D.10) yields

$$
\begin{gathered}
-\frac{2 \sqrt{(l(l+1)}}{r} \frac{G^{2}}{2}(J-L)^{\prime} R_{4 l 1 l} \\
-\frac{2 \sqrt{(l(l+1)}}{r} \frac{B^{2}}{\left(E_{1}+M_{1}\right)\left(E_{2}+M_{2}\right)} \frac{i G^{2}}{2}(J-L)^{\prime} R_{1 l 1 l}=0,
\end{gathered}
$$


or

$$
R_{4 l 1 l}+\frac{B^{2}}{\left(E_{1}+M_{1}\right)\left(E_{2}+M_{2}\right)} R_{1 l 1 l}=0 .
$$

(An independent evaluation of this for $l=0$ (see (D.47)) shows that this is also true for $l=0)$. The triplet $(j=l)$ component of $(D .10)$ yields

$$
R_{4 l 0 l}-\frac{B^{2}}{\left(E_{1}+M_{1}\right)\left(E_{2}+M_{2}\right)} R_{1 l 0 l}=0 .
$$

These simple algebraic relations between the upper-upper and lowerlower components of the wave function allow us to decouple the radial part of $(4.21 b)$ from that of $(4.21 a)$. Eqs. (D.27,28) then take the form

$$
\psi_{4}=F(r)(-1)^{s} \psi_{1}, s=0,1
$$

In Refs.(4-5) we assumed that this result would also hold for $s=1, j=l \pm$ 1. However, this assumption was erroneous since the operator $L \cdot\left(\sigma_{1}-\sigma_{2}\right)$ vanishes on $j=l \pm 1$ states, that is

$$
<l s=1 j=l \pm 1 m \mid L \cdot\left(\sigma_{1}-\sigma_{2}\right)=0
$$

Since our earlier derivation of (D.27,D.28) turned out to be limited to $j=l$ states, we present a new derivation based on the sum of Eq.(D.6) and Eq.(D.7) rather than the difference ${ }^{79}$. In addition to confirming our earlier result $(\mathrm{D} .27,28)$ this derivation leads to decoupling for the ${ }^{3} P_{0}$ state (although not for any other $j=l \pm 1$ states). The sum of Eqs.(D.6) and (D.7) is

$$
\begin{gathered}
\left\{\frac{G^{2}}{2}(J-L)^{\prime}\left[2 i \hat{r} \cdot p-\frac{L \cdot\left(\sigma_{1}+\sigma_{2}\right)}{r}\right]\right. \\
\left.+\frac{G^{2}}{2}(J-L)^{\prime} \mathcal{G}^{\prime}\left(1-\sigma_{1} \cdot \sigma_{2}+\sigma_{1} \cdot \hat{r} \sigma_{2} \cdot \hat{r}\right)+\frac{B^{2}}{\left(E_{1}+M_{1}\right)\left(E_{2}+M_{2}\right)} \frac{G^{2}}{2}(\partial J-\partial L)^{2}\right\} \psi_{4}
\end{gathered}
$$




$$
\begin{aligned}
& +\left\{\frac{\left(E_{2}-M_{2}\right)}{\left(E_{1}+M_{1}\right)} \frac{i G^{2}}{2}(J-L)^{\prime} \hat{r} \cdot \sigma_{1}\left(\sigma_{2} \cdot p\right)+\frac{\left(E_{1}-M_{1}\right)}{\left(E_{2}+M_{2}\right)} \frac{i G^{2}}{2}(J-L)^{\prime} \hat{r} \cdot \sigma_{2}\left(\sigma_{1} \cdot p\right)\right. \\
& \left.+\frac{B^{2}}{\left(E_{1}+M_{1}\right)\left(E_{2}+M_{2}\right)} \frac{G^{2}}{2}(J-L)^{\prime} \sigma_{1} \cdot \sigma_{2} \mathcal{G}^{\prime}+\frac{G^{2}}{2}\left((J-L)^{\prime}\right)^{2} \sigma_{1} \cdot \hat{r} \sigma_{2} \cdot \hat{r}\right\} \psi_{1}=0 .
\end{aligned}
$$

Notice that this equation is not an algebraic equation because of the $i \hat{r} \cdot p$ term. Again we use Dirac's trick and multiply this by $\sigma_{1} \cdot \hat{r} \sigma_{2} \cdot \hat{r}$ leading to

$$
\begin{gathered}
\left\{\sigma_{1} \cdot \hat{r} \sigma_{2} \cdot \hat{r} \frac{G^{2}}{2}(J-L)^{\prime}\left[2 i \hat{r} \cdot p-\frac{L \cdot\left(\sigma_{1}+\sigma_{2}\right)}{r}\right]\right. \\
\left.+\frac{G^{2}}{2}(J-L)^{\prime} \mathcal{G}^{\prime} \sigma_{1} \cdot \sigma_{2}+\sigma_{1} \cdot \hat{r} \sigma_{2} \cdot \hat{r} \frac{B^{2}}{\left(E_{1}+M_{1}\right)\left(E_{2}+M_{2}\right)} \frac{G^{2}}{2}(\partial J-\partial L)^{2}\right\} \psi_{4} \\
+\left\{\frac{\left(E_{2}-M_{2}\right)}{\left(E_{1}+M_{1}\right)} \frac{G^{2}}{2}(J-L)^{\prime}\left(i \hat{r} \cdot p-\frac{L \cdot \sigma_{2}}{r}\right)+\frac{\left(E_{1}-M_{1}\right)}{\left(E_{2}+M_{2}\right)} \frac{G^{2}}{2}(J-L)^{\prime}\left(i \hat{r} p-\frac{L \cdot \sigma_{1}}{r}\right)\right. \\
\left.+\frac{B^{2}}{\left(E_{1}+M_{1}\right)\left(E_{2}+M_{2}\right)} \frac{G^{2}}{2}(J-L)^{\prime}\left(1-\sigma_{1} \cdot \sigma_{2}+\sigma_{1} \cdot \hat{r} \sigma_{2} \cdot \hat{r}\right) \mathcal{G}^{\prime}+\frac{G^{2}}{2}\left((J-L)^{\prime}\right)^{2}\right\} \psi_{1}=0 .
\end{gathered}
$$

We combine Darwin and spin-orbit terms and cancel common factors to obtain

$$
\begin{gathered}
\sigma_{1} \cdot \hat{r} \sigma_{2} \cdot \hat{r}\left\{2 i \hat{r} \cdot p-\frac{L \cdot\left(\sigma_{1}+\sigma_{2}\right)}{r}+\mathcal{G}^{\prime} \sigma_{1} \cdot \hat{r} \sigma_{2} \cdot \hat{r} \sigma_{1} \cdot \sigma_{2}\right. \\
\left.+\frac{B^{2}}{\left(E_{1}+M_{1}\right)\left(E_{2}+M_{2}\right)}(J-L)^{\prime}\right\} \psi_{4} \\
=-\left\{\frac{B^{2}}{\left(E_{1}+M_{1}\right)\left(E_{2}+M_{2}\right)}\left[\left(2 i \hat{r} \cdot p-\frac{L \cdot\left(\sigma_{1}+\sigma_{2}\right)}{r}+\sigma_{1} \cdot \hat{r} \sigma_{2} \cdot \hat{r} \sigma_{1} \cdot \sigma_{2} \mathcal{G}^{\prime}\right]+(J-L)^{\prime}\right\} \psi_{1}\right. \\
=0 .
\end{gathered}
$$

Before doing an angular momentum decomposition on this equation, based on the form of this equation, we rewrite $\psi_{4}$ as

$$
\psi_{4}=-\sigma_{1} \cdot \hat{r} \sigma_{2} \cdot \hat{r} \frac{B^{2}}{\left(E_{1}+M_{1}\right)\left(E_{2}+M_{2}\right)} \psi_{1}+\phi_{4} \text {. }
$$


To simplify Eq.(D.33) we use $\left\{i \hat{r} \cdot p, \sigma_{1} \cdot \sigma_{2}\right\}=0$ and Eq.(D.34) to write

$$
\begin{gathered}
i \hat{r} \cdot p \psi_{4}=-\sigma_{1} \cdot \hat{r} \sigma_{2} \cdot \hat{r} \frac{B^{2}}{\left(E_{1}+M_{1}\right)\left(E_{2}+M_{2}\right)} i \hat{r} \cdot p \psi_{1} \\
-\sigma_{1} \cdot \hat{r} \sigma_{2} \cdot \hat{r}\left[E_{1}^{3} M_{1}+E_{2}^{3} M_{2}+E_{1} M_{1}^{2}+E_{2} M_{2}^{3}+2 E_{2}^{2} M_{2}^{2}+2 E_{1}^{2} M_{1}^{2}-E_{2}^{2} M_{1}^{2}\right. \\
\left.-E_{1}^{2} M_{2}^{2}+2 M_{1} M_{2} E_{1} E_{2}\right] /\left[\left(E_{1}+M_{1}\right)^{2}\left(E_{2}+M_{2}\right)^{2}\right](J-L)^{\prime} \psi_{1} \\
+\hat{r} \cdot p \phi_{4} .
\end{gathered}
$$

This form plus the three identities

$$
\sigma_{1} \cdot \hat{r} \sigma_{2} \cdot \hat{r} \hat{r} \cdot p \sigma_{1} \cdot \hat{r} \sigma_{2} \cdot \hat{r}=\hat{r} \cdot p
$$

$\sigma_{1} \cdot \hat{r} \sigma_{2} \cdot \hat{r} \sigma_{1} \cdot \hat{r} \sigma_{2} \cdot \hat{r} \sigma_{1} \cdot \sigma_{2} \sigma_{1} \cdot \hat{r} \sigma_{2} \cdot \hat{r}=\sigma_{1} \cdot \sigma_{2} \sigma_{1} \cdot \hat{r} \sigma_{2} \cdot \hat{r}=\sigma_{1} \cdot \hat{r} \sigma_{2} \cdot \hat{r} \sigma_{1} \cdot \sigma_{2}$,

$\sigma_{1} \cdot \hat{r} \sigma_{2} \cdot \hat{r} L \cdot\left(\sigma_{1}+\sigma_{2}\right) \sigma_{1} \cdot \hat{r} \sigma_{2} \cdot \hat{r}=L \cdot\left(\sigma_{1}+\sigma_{2}\right)+2 S_{T}+2 \sigma_{1} \cdot \hat{r} \sigma_{2} \cdot \hat{r} L \cdot\left(\sigma_{1}+\sigma_{2}\right)$,

lead (after numerous cancellations) to

$$
\begin{gathered}
\sigma_{1} \cdot \hat{r} \sigma_{2} \cdot \hat{r}\left\{2 i \hat{r} \cdot p-\frac{L \cdot\left(\sigma_{1}+\sigma_{2}\right)}{r}+\mathcal{G}^{\prime} \sigma_{1} \cdot \hat{r} \sigma_{2} \cdot \hat{r} \sigma_{1} \cdot \sigma_{2}\right. \\
\left.+\frac{B^{2}}{\left(E_{1}+M_{1}\right)\left(E_{2}+M_{2}\right)}(J-L)^{\prime}\right\} \phi_{4} \\
-\frac{\left[2 S_{T}+2 \sigma_{1} \cdot \hat{r} \sigma_{2} \cdot \hat{r} L \cdot\left(\sigma_{1}+\sigma_{2}\right)\right]}{r} \frac{B^{2}}{\left(E_{1}+M_{1}\right)\left(E_{2}+M_{2}\right)} \psi_{1}=0 .
\end{gathered}
$$

This equation is coupled to our main Schrödinger equation (4.21a-b). To simplify the form of the coupling that comes from insertion of (D.34) into Eq. $(4.21 \mathrm{a})$ we need

$$
-\sigma_{1} \cdot \sigma_{2} \sigma_{1} \cdot \hat{r} \sigma_{2} \cdot \hat{r}=-1+\sigma_{1} \cdot \sigma_{2}-\sigma_{1} \cdot \hat{r} \sigma_{2} \cdot \hat{r}=-1+\frac{2}{3} \sigma_{1} \cdot \sigma_{2}-\frac{1}{3} S_{T}
$$




$$
-S_{T} \sigma_{1} \cdot \hat{r} \sigma_{2} \cdot \hat{r}=-2-\sigma_{1} \cdot \sigma_{2}+\sigma_{1} \cdot \hat{r} \sigma_{2} \cdot \hat{r}=-2-\frac{2}{3} \sigma_{1} \cdot \sigma_{2}+\frac{1}{3} S_{T}
$$

Then Eq.(4.21a) becomes

$$
\begin{aligned}
& \left\{p^{2}+2 m_{w} S+S^{2}+2 \epsilon_{w} \mathcal{A}-\mathcal{A}^{2}+2 \epsilon_{w} \mathcal{V}-\mathcal{V}^{2}+\frac{1}{4}(\partial J-\partial L)^{2}\right. \\
& +i l n^{\prime} \chi_{1} \hat{r} \cdot p-\frac{\ln ^{\prime} \chi_{1}}{r} L \cdot \sigma_{1}+i \ln ^{\prime} \chi_{2} \hat{r} \cdot p-\frac{\ln ^{\prime} \chi_{2}}{r} L \cdot \sigma_{2} \\
& \left.-\frac{1}{2} \partial^{2} \mathcal{G}+\frac{3}{4}\left(\mathcal{G}^{\prime}\right)^{2}+\frac{1}{2} \ln ^{\prime} \chi_{1} \chi_{2} \mathcal{G}^{\prime}\right) \\
& -\left[\frac{1}{2} \ln ^{\prime} \chi_{1} \chi_{2}(J-L)^{\prime}+\frac{1}{2} \mathcal{G}^{\prime}(J-L)^{\prime}-\frac{1}{2} \partial^{2}(J-L)+\frac{(J-L)^{\prime}}{r}\right] \frac{B^{2}}{\left(E_{1}+M_{1}\right)\left(E_{2}+M_{2}\right)} \\
& +\left[\left[\frac{1}{3} \partial^{2} \mathcal{G}-\frac{1}{2}\left(\mathcal{G}^{\prime}\right)^{2}-\frac{1}{3} \ln ^{\prime} \chi_{1} \chi_{2} \mathcal{G}^{\prime}\right]\right. \\
& \left.\left.+\left[+\frac{1}{3} \mathcal{G}^{\prime}(J-L)^{\prime}-\frac{1}{3} \frac{(J-L)^{\prime}}{r}\right)\right] \frac{B^{2}}{\left(E_{1}+M_{1}\right)\left(E_{2}+M_{2}\right)}\right] \sigma_{1} \cdot \sigma_{2} \\
& {\left[\left[-\frac{1}{6}\left(\mathcal{G}^{\prime \prime} \frac{\mathcal{G}^{\prime}}{r}\right)+\frac{1}{6} \ln ^{\prime} \chi_{1} \chi_{2} \mathcal{G}^{\prime}\right]\right.} \\
& \left.\left.\left.-\left[\frac{1}{6} \mathcal{G}^{\prime}(J-L)^{\prime}-\frac{1}{6} \frac{(J-L)^{\prime}}{r}\right)\right] \frac{B^{2}}{\left(E_{1}+M_{1}\right)\left(E_{2}+M_{2}\right)}\right] S_{T}\right\} \psi_{1} \\
& +\left\{\left[+\frac{1}{6} \ln ^{\prime} \chi_{1} \chi_{2}(J-L)^{\prime}+\frac{1}{2} \mathcal{G}^{\prime}(J-L)^{\prime}-\frac{1}{6} \partial^{2}(J-L)\right] \sigma_{1} \cdot \sigma_{2}\right. \\
& \left.\left[+\frac{1}{6} \ln ^{\prime} \chi_{1} \chi_{2}(J-L)^{\prime}-\frac{1}{6}\left((J-L)^{\prime \prime}-\frac{(J-L)^{\prime}}{r}\right)\right] S_{T}\right\} \phi_{4}=b^{2}(w) \psi_{1}
\end{aligned}
$$

Since all of the energy denominators in Eq.(D.42) are of form $E_{i}+M_{i}>$ 0 , we can eliminate the non-hermitian term $i \hat{r} \cdot p$ without producing any squared denominators that vanish by making the scale change $\psi=$ $\sqrt{\chi_{1} \chi_{2}} \Psi$. Then our equation (D.42) becomes

$$
\left\{p^{2}+2 m_{w} S+S^{2}+2 \epsilon_{w} \mathcal{A}-\mathcal{A}^{2}+2 \epsilon_{w} \mathcal{V}-\mathcal{V}^{2}+\frac{1}{4}(\partial J-\partial L)^{2}\right.
$$




$$
\begin{gathered}
-\frac{1}{2} \partial^{2} \ln \left(\chi_{1} \chi_{2} G\right)+\frac{3}{4}\left(\mathcal{G}^{\prime}\right)^{2}+\frac{1}{2} \ln ^{\prime} \chi_{1} \chi_{2} \mathcal{G}^{\prime}+\frac{1}{4}\left(\ln ^{\prime} \chi_{1} \chi_{2}\right)^{2} \\
-\left[\frac{1}{2} l^{\prime} \chi_{1} \chi_{2}(J-L)^{\prime}+\frac{1}{2} \mathcal{G}^{\prime}(J-L)^{\prime}-\frac{1}{2} \partial^{2}(J-L)+\frac{(J-L)^{\prime}}{r}\right] \frac{B^{2}}{\left(E_{1}+M_{1}\right)\left(E_{2}+M_{2}\right)} \\
-\frac{1}{2} \frac{l^{\prime} \chi_{1} \chi_{2}}{r} L \cdot\left(\sigma_{1}+\sigma_{2}\right)-\frac{1}{2} \frac{l^{\prime}\left(\chi_{1} / \chi_{2}\right)}{r} L \cdot\left(\sigma_{1}-\sigma_{2}\right) \\
+\left[\left[\frac{1}{3} \partial^{2} \mathcal{G}-\frac{1}{2}\left(\mathcal{G}^{\prime}\right)^{2}-\frac{1}{3} l^{\prime} \chi_{1} \chi_{2} \mathcal{G}^{\prime}\right]\right. \\
\left.\left.+\left[\frac{1}{3} \mathcal{G}^{\prime}(J-L)^{\prime}-\frac{1}{3} \frac{(J-L)^{\prime}}{r}\right)\right] \frac{B^{2}}{\left(E_{1}+M_{1}\right)\left(E_{2}+M_{2}\right)}\right] \sigma_{1} \cdot \sigma_{2} \\
\left.\left.\left.-\left[\frac{1}{6} \mathcal{G}^{\prime}(J-L)^{\prime}-\frac{1}{6} \frac{(J-L)^{\prime}}{r}\right)\right] \frac{\mathcal{G}^{\prime}}{r}\right)+\frac{1}{6} l n^{\prime} \chi_{1} \chi_{2} \mathcal{G}^{\prime}\right] \\
+\left\{\left[+\frac{1}{6} l n^{\prime} \chi_{1} \chi_{2}(J-L)^{\prime}+\frac{1}{2} \mathcal{G}^{\prime}(J-L)^{\prime}-\frac{1}{6} \partial^{2}(J-L)\right] \sigma_{1} \cdot \sigma_{2}\right. \\
\left.\left[+\frac{1}{6} l_{n}^{\prime} \chi_{1} \chi_{2}(J-L)^{\prime}-\frac{1}{6}\left((J-L)^{\prime \prime}-\frac{(J-L)^{\prime}}{r}\right)\right] S_{T}\right\} \Phi_{4}=b^{2}(w) \Psi_{1},(D .43)
\end{gathered}
$$

and is coupled to

$$
\begin{gathered}
\sigma_{1} \cdot \hat{r} \sigma_{2} \cdot \hat{r}\left\{2 i \hat{r} \cdot p-\frac{L \cdot\left(\sigma_{1}+\sigma_{2}\right)}{r}+\ln ^{\prime} \chi_{1} \chi_{2}+\mathcal{G}^{\prime} \sigma_{1} \cdot \hat{r} \sigma_{2} \cdot \hat{r} \sigma_{1} \cdot \sigma_{2}\right. \\
\left.+\frac{B^{2}}{\left(E_{1}+M_{1}\right)\left(E_{2}+M_{2}\right)}(J-L)^{\prime}\right\} \Phi_{4} \\
-\frac{\left[2 S_{T}+2 \sigma_{1} \cdot \hat{r} \sigma_{2} \cdot \hat{r} L \cdot\left(\sigma_{1}+\sigma_{2}\right)\right]}{r} \frac{B^{2}}{\left(E_{1}+M_{1}\right)\left(E_{2}+M_{2}\right)} \Psi_{1}=0
\end{gathered}
$$

in which

$$
\phi_{4}=\sqrt{\chi_{1} \chi_{2}} \Phi_{4}
$$


and

$$
\psi_{1}=\sqrt{\chi_{1} \chi_{2}} \Psi_{1}
$$

We now examine the angular momentum decomposition of our set of coupled equations. Let $u_{j l s}(r)$ be the radial wave function associated with $\Psi_{1}$ and let $v_{j l s}(r)$ be the radial wave function associated with $\Phi_{4}$. Taking the $<r l s j m \mid$ component of (D.44) first for $s=0, l=j$, we find that since $<r j 0 j m\left|\sigma_{1} \cdot \hat{r} \sigma_{2} \cdot \hat{r}=-<r j 0 j m\right|$, Eq.(D.44) becomes

$$
-\left\{2 \frac{d}{d r}+\ln ^{\prime} \chi_{1} \chi_{2}+3 \mathcal{G}^{\prime}+\frac{B^{2}}{\left(E_{1}+M_{1}\right)\left(E_{2}+M_{2}\right)}(J-L)^{\prime}\right\} v_{j 0 j}=0 .
$$

The only solution to this and Eq.(D.10) is $v_{j 0 j}=0$. Note that this solution of Eq.(D.34) corresponds to our earlier solution Eq.(D.27). Our main equation Eq.(D.43) then becomes

$$
\begin{gathered}
\left\{-\frac{d^{2}}{d r^{2}}+\frac{j(j+1)}{r^{2}}+2 m_{w} S+S^{2}+2 \epsilon_{w} \mathcal{A}-\mathcal{A}^{2}+2 \epsilon_{w} \mathcal{V}-\mathcal{V}^{2}+\frac{1}{4}(\partial J-\partial L)^{2}\right. \\
-\frac{1}{2} \partial^{2} \ln \left(\chi_{1} \chi_{2} G^{3}\right)+\frac{9}{4}\left(\mathcal{G}^{\prime}\right)^{2}+\frac{3}{2} \ln ^{\prime} \chi_{1} \chi_{2} \mathcal{G}^{\prime}+\frac{1}{4}\left(\ln ^{\prime} \chi_{1} \chi_{2}\right)^{2} \\
\left.-\left[\frac{1}{2} \ln ^{\prime} \chi_{1} \chi_{2}(J-L)^{\prime}+\frac{3}{2} \mathcal{G}^{\prime}(J-L)^{\prime}-\frac{1}{2} \partial^{2}(J-L)\right] \frac{B^{2}}{\left(E_{1}+M_{1}\right)\left(E_{2}+M_{2}\right)}\right\} u_{j 0 j} \\
-\sqrt{(j(j+1)} \frac{\ln ^{\prime}\left(\chi_{1} / \chi_{2}\right)}{r} u_{j 1 j}=b^{2}(w) u_{j 0 j},
\end{gathered}
$$

in which the effect of the coupling to the lower-lower component is accounted for by the terms in square brackets. Next we consider the case of $s=1, j=l$ for which Eq.(D.44) becomes

$$
2\left\{2 \frac{d}{d r}+\frac{2}{r}+\ln ^{\prime} \chi_{1} \chi_{2}+2 \mathcal{G}^{\prime}+\frac{B^{2}}{\left(E_{1}+M_{1}\right)\left(E_{2}+M_{2}\right)}(J-L)^{\prime}\right\} v_{j 1 j}=0
$$


which again has the simple solution $v_{j 1 j}=0$, in agreement with our earlier solution Eq.(D.28). Our main equation Eq.(D.43) then becomes

$$
\begin{gathered}
\left\{-\frac{d^{2}}{d r^{2}}+\frac{j(j+1)}{r^{2}}+2 m_{w} S+S^{2}+2 \epsilon_{w} \mathcal{A}-\mathcal{A}^{2}+2 \epsilon_{w} \mathcal{V}-\mathcal{V}^{2}+\frac{1}{4}(\partial J-\partial L)^{2}\right. \\
-\frac{1}{2} \partial^{2} \ln \left(\chi_{1} \chi_{2} G\right)+\frac{1}{4}\left(\mathcal{G}^{\prime}\right)^{2}+\frac{1}{6} \ln ^{\prime} \chi_{1} \chi_{2} \mathcal{G}^{\prime}+\frac{1}{4}\left(\ln ^{\prime} \chi_{1} \chi_{2}\right)^{2}+\frac{1}{2} \frac{\mathcal{G}^{\prime}}{r} \\
-\left[\frac{1}{2} \ln ^{\prime} \chi_{1} \chi_{2}(J-L)^{\prime}+\frac{1}{2} \mathcal{G}^{\prime}(J-L)^{\prime}-\frac{1}{2} \partial^{2}(J-L)+\frac{(J-L)^{\prime}}{r}\right] \frac{B^{2}}{\left(E_{1}+M_{1}\right)\left(E_{2}+M_{2}\right)} \\
\left.+\frac{\ln ^{\prime} \chi_{1} \chi_{2}}{r}\right\} u_{j 1 j}-\sqrt{(j(j+1)} \frac{\ln ^{\prime}\left(\chi_{1} / \chi_{2}\right)}{r} u_{j 0 j}=b^{2}(w) u_{j 1 j} .
\end{gathered}
$$

Note that these two Schrödinger equations (Eqs.(D.48) and (D.50)) decouple completely for equal mass systems and that in both cases the effects of the coupling to the lower-lower component is accounted for by the terms in square brackets.

Next we examine the $s=1, l=j \pm 1$ equations. Our auxiliary equation Eq.(D.44) becomes the two coupled equations

$$
\begin{gathered}
\left.-\frac{1}{2 j+1}\left[2 \frac{d}{d r}+\ln ^{\prime} \chi_{1} \chi_{2}+2 \frac{j+2}{r}-(2 j+1) \mathcal{G}^{\prime}+\frac{B^{2}}{\left(E_{1}+M_{1}\right)\left(E_{2}+M_{2}\right)}(J-L)^{\prime}\right\}\right] v_{j+11 j} \\
+2 \frac{\sqrt{(j(j+1)}}{2 j+1}\left[2 \frac{d}{d r}-2 \frac{j-1}{r}\right] v_{j-11 j} \\
-4 \sqrt{(j(j+1)} \frac{B^{2}}{\left(E_{1}+M_{1}\right)\left(E_{2}+M_{2}\right)} u_{j-11 j}=0,
\end{gathered}
$$

and

$$
\begin{gathered}
\left.\frac{1}{2 j+1}\left[2 \frac{d}{d r}+\ln ^{\prime} \chi_{1} \chi_{2}-2 \frac{j-1}{r}+(2 j+1) \mathcal{G}^{\prime}+\frac{B^{2}}{\left(E_{1}+M_{1}\right)\left(E_{2}+M_{2}\right)}(J-L)^{\prime}\right\}\right] v_{j-11 j} \\
+2 \frac{\sqrt{(j(j+1)}}{2 j+1}\left[2 \frac{d}{d r}+2 \frac{j+2}{r}\right] v_{j+11 j}
\end{gathered}
$$




$$
+4 \sqrt{(j(j+1)} \frac{B^{2}}{\left(E_{1}+M_{1}\right)\left(E_{2}+M_{2}\right)} u_{j+11 j}=0 .
$$

Notice that for $j=0$ the $v$ wave functions again vanish so that we obtain decoupling for the ${ }^{3} P_{0}$ states. For the general $j=l \pm 1>0$ case the above two equations are first order differential equations coupled to the two coupled Schrödinger equations

$$
\begin{aligned}
& \left\{-\frac{d^{2}}{d r^{2}}+\frac{(j+1)(j+2)}{r^{2}}+2 m_{w} S+S^{2}+2 \epsilon_{w} \mathcal{A}-\mathcal{A}^{2}+2 \epsilon_{w} \mathcal{V}-\mathcal{V}^{2}+\frac{1}{4}(\partial J-\partial L)^{2}\right. \\
& \left.-\frac{1}{2} \partial^{2} \ln \left(\chi_{1} \chi_{2} G^{1 / 3}\right)+\frac{1}{4}\left(\mathcal{G}^{\prime}\right)^{2}+\frac{1}{6} \ln ^{\prime} \chi_{1} \chi_{2} \mathcal{G}^{\prime}\right)+\frac{1}{4}\left(\ln ^{\prime} \chi_{1} \chi_{2}\right)^{2} \\
& -\left[\frac{1}{2} \ln ^{\prime} \chi_{1} \chi_{2}(J-L)^{\prime}+\frac{1}{6} \mathcal{G}^{\prime}(J-L)^{\prime}-\frac{1}{2} \partial^{2}(J-L)-\frac{4(J-L)^{\prime}}{3 r}\right] \frac{B^{2}}{\left(E_{1}+M_{1}\right)\left(E_{2}+M_{2}\right)} \\
& +(j+2) \frac{\ln ^{\prime} \chi_{1} \chi_{2}}{r} \\
& -\frac{2 j+4}{2 j+1}\left[\left[-\frac{1}{6}\left(\mathcal{G}^{\prime \prime}-\frac{\mathcal{G}^{\prime}}{r}\right)+\frac{1}{6} \ln ^{\prime} \chi_{1} \chi_{2} \mathcal{G}^{\prime}\right]\right. \\
& \left.\left.\left.-\left[\frac{1}{6} \mathcal{G}^{\prime}(J-L)^{\prime}-\frac{1}{6} \frac{(J-L)^{\prime}}{r}\right)\right] \frac{B^{2}}{\left(E_{1}+M_{1}\right)\left(E_{2}+M_{2}\right)}\right]\right\} u_{j+11 j} \\
& +6 \frac{\sqrt{(j(j+1)}}{2 j+1}\left[\left[-\frac{1}{6}\left(\mathcal{G}^{\prime \prime}-\frac{\mathcal{G}^{\prime}}{r}\right)+\frac{1}{6} \ln ^{\prime} \chi_{1} \chi_{2} \mathcal{G}^{\prime}\right]\right. \\
& \left.\left.\left.-\left[\frac{1}{6} \mathcal{G}^{\prime}(J-L)^{\prime}-\frac{1}{6} \frac{(J-L)^{\prime}}{r}\right)\right] \frac{B^{2}}{\left(E_{1}+M_{1}\right)\left(E_{2}+M_{2}\right)}\right]\right\} u_{j-11 j} \\
& +\left\{\left[+\frac{1}{6} \ln ^{\prime} \chi_{1} \chi_{2}(J-L)^{\prime}+\frac{1}{2} \mathcal{G}^{\prime}(J-L)^{\prime}-\frac{1}{6} \partial^{2}(J-L)\right]\right. \\
& \left.-\frac{2 j+4}{2 j+1}\left[+\frac{1}{6} l^{\prime} \chi_{1} \chi_{2}(J-L)^{\prime}-\frac{1}{6}\left((J-L)^{\prime \prime}-\frac{(J-L)^{\prime}}{r}\right)\right]\right\} v_{j+11 j} \\
& \left.+\frac{\sqrt{(j(j+1)}}{2 j+1}\left[+\frac{1}{6} l n^{\prime} \chi_{1} \chi_{2}(J-L)^{\prime}-\frac{1}{6}\left((J-L)^{\prime \prime}-\frac{(J-L)^{\prime}}{r}\right)\right]\right\} v_{j-11 j}=b^{2}(w) u_{j+11 j}
\end{aligned}
$$


and

$$
\begin{aligned}
& \left\{-\frac{d^{2}}{d r^{2}}+\frac{(j-1) j}{r^{2}}+2 m_{w} S+S^{2}+2 \epsilon_{w} \mathcal{A}-\mathcal{A}^{2}+2 \epsilon_{w} \mathcal{V}-\mathcal{V}^{2}+\frac{1}{4}(\partial J-\partial L)^{2}\right. \\
& \left.-\frac{1}{2} \partial^{2} \ln \left(\chi_{1} \chi_{2} G^{1 / 3}\right)+\frac{1}{4}\left(\mathcal{G}^{\prime}\right)^{2}+\frac{1}{6} \ln ^{\prime} \chi_{1} \chi_{2} \mathcal{G}^{\prime}\right)+\frac{1}{4}\left(\ln ^{\prime} \chi_{1} \chi_{2}\right)^{2} \\
& -\left[\frac{1}{2} \ln ^{\prime} \chi_{1} \chi_{2}(J-L)^{\prime}+\frac{1}{6} \mathcal{G}^{\prime}(J-L)^{\prime}-\frac{1}{2} \partial^{2}(J-L)+\frac{4(J-L)^{\prime}}{3 r}\right] \frac{B^{2}}{\left(E_{1}+M_{1}\right)\left(E_{2}+M_{2}\right)} \\
& -(j-1) \frac{\ln \chi^{\prime} \chi_{1} \chi_{2}}{r} \\
& -\frac{2 j-2}{2 j+1}\left[\left[-\frac{1}{6}\left(\mathcal{G}^{\prime \prime}-\frac{\mathcal{G}^{\prime}}{r}\right)+\frac{1}{6} \ln ^{\prime} \chi_{1} \chi_{2} \mathcal{G}^{\prime}\right]\right. \\
& \left.\left.\left.-\left[\frac{1}{6} \mathcal{G}^{\prime}(J-L)^{\prime}-\frac{1}{6} \frac{(J-L)^{\prime}}{r}\right)\right] \frac{B^{2}}{\left(E_{1}+M_{1}\right)\left(E_{2}+M_{2}\right)}\right]\right\} u_{j-11 j} \\
& +6 \frac{\sqrt{(j(j+1)}}{2 j+1}\left[\left[-\frac{1}{6}\left(\mathcal{G}^{\prime \prime}-\frac{\mathcal{G}^{\prime}}{r}\right)+\frac{1}{6} \ln ^{\prime} \chi_{1} \chi_{2} \mathcal{G}^{\prime}\right]\right. \\
& \left.\left.\left.-\left[\frac{1}{6} \mathcal{G}^{\prime}(J-L)^{\prime}-\frac{1}{6} \frac{(J-L)^{\prime}}{r}\right)\right] \frac{B^{2}}{\left(E_{1}+M_{1}\right)\left(E_{2}+M_{2}\right)}\right]\right\} u_{j+11 j} \\
& +\left\{\left[+\frac{1}{6} l^{\prime} \chi_{1} \chi_{2}(J-L)^{\prime}+\frac{1}{2} \mathcal{G}^{\prime}(J-L)^{\prime}-\frac{1}{6} \partial^{2}(J-L)\right]\right. \\
& \left.-\frac{2 j-2}{2 j+1}\left[+\frac{1}{6} \ln ^{\prime} \chi_{1} \chi_{2}(J-L)^{\prime}-\frac{1}{6}\left((J-L)^{\prime \prime}-\frac{(J-L)^{\prime}}{r}\right)\right]\right\} v_{j-11 j} \\
& \left.+\frac{\sqrt{(j(j+1)}}{2 j+1}\left[+\frac{1}{6} l n^{\prime} \chi_{1} \chi_{2}(J-L)^{\prime}-\frac{1}{6}\left((J-L)^{\prime \prime}-\frac{(J-L)^{\prime}}{r}\right)\right]\right\} v_{j+11 j}=b^{2}(w) u_{j-11 j}
\end{aligned}
$$

We would need to solve these four simultaneous equations in the general case. Note that they would provide an alternative to the set of equations (6.3a-d) used in the text. For the ${ }^{3} P_{0}$ our main equation Eq.(D.43) simplifies to

$$
\left\{-\frac{d^{2}}{d r^{2}}+\frac{2}{r^{2}}+2 m_{w} S+S^{2}+2 \epsilon_{w} \mathcal{A}-\mathcal{A}^{2}+2 \epsilon_{w} \mathcal{V}-\mathcal{V}^{2}+\frac{1}{4}(\partial J-\partial L)^{2}\right.
$$




$$
\begin{gathered}
-\frac{1}{2} \partial^{2} \ln \left(\chi_{1} \chi_{2} G^{-1}\right)+\frac{1}{4}\left(\mathcal{G}^{\prime}\right)^{2}-\frac{1}{2} \ln ^{\prime} \chi_{1} \chi_{2} \mathcal{G}^{\prime}+\frac{1}{4}\left(\ln ^{\prime} \chi_{1} \chi_{2}\right)^{2}-2 \frac{\mathcal{G}^{\prime}}{r} \\
-\left[\frac{1}{2} \ln ^{\prime} \chi_{1} \chi_{2}(J-L)^{\prime}-\frac{1}{2} \mathcal{G}^{\prime}(J-L)^{\prime}-\frac{1}{2} \partial^{2}(J-L)\right. \\
\left.+\frac{2(J-L)^{\prime}}{3 r}\right] \frac{B^{2}}{\left(E_{1}+M_{1}\right)\left(E_{2}+M_{2}\right)} \\
\left.+2 \frac{\ln ^{\prime} \chi_{1} \chi_{2}}{r}\right\} u_{110}=b^{2}(w) u_{110} .
\end{gathered}
$$

For QED $(L=0, J=-\mathcal{G}$ and $S=0=\mathcal{V})$, our $j=l$ (Eqs.(D.48) and (D.50)) equations become

$$
\begin{gathered}
\left\{-\frac{d^{2}}{d r^{2}}+\frac{j(j+1)}{r^{2}}+2 \epsilon_{w} \mathcal{A}-\mathcal{A}^{2}\right. \\
-\frac{1}{2} \partial^{2} \ln \left(\chi_{1} \chi_{2} G^{3}\right)+\frac{5}{2}\left(\mathcal{G}^{\prime}\right)^{2}+\frac{3}{2} \ln ^{\prime} \chi_{1} \chi_{2} \mathcal{G}^{\prime}+\frac{1}{4}\left(\ln ^{\prime} \chi_{1} \chi_{2}\right)^{2} \\
\left.+\left[\frac{1}{2} \ln ^{\prime} \chi_{1} \chi_{2} \mathcal{G}^{\prime}+\frac{3}{2}\left(\mathcal{G}^{\prime}\right)^{2}-\frac{1}{2} \partial^{2} \mathcal{G}\right] \frac{B^{2}}{\left(E_{1}+m_{1}\right)\left(E_{2}+m_{2}\right)}\right\} u_{j 0 j} \\
-\sqrt{(j(j+1)} \frac{\ln ^{\prime}\left(\chi_{1} / \chi_{2}\right)}{r} u_{j 1 j}=b^{2}(w) u_{j 0 j},
\end{gathered}
$$

and

$$
\begin{gathered}
\left\{-\frac{d^{2}}{d r^{2}}+\frac{j(j+1)}{r^{2}}+2 \epsilon_{w} \mathcal{A}-\mathcal{A}^{2}\right. \\
-\frac{1}{2} \partial^{2} \ln \left(\chi_{1} \chi_{2} G\right)+\frac{1}{2}\left(\mathcal{G}^{\prime}\right)^{2}+\frac{1}{2} \ln ^{\prime} \chi_{1} \chi_{2} \mathcal{G}^{\prime}+\frac{1}{4}\left(\ln ^{\prime} \chi_{1} \chi_{2}\right)^{2}+\frac{\mathcal{G}^{\prime}}{r} \\
+\left[\frac{1}{2} \ln ^{\prime} \chi_{1} \chi_{2} \mathcal{G}^{\prime}+\frac{1}{2}\left(\mathcal{G}^{\prime}\right)^{2}-\frac{1}{2} \partial^{2} \mathcal{G}+\frac{\mathcal{G}^{\prime}}{r}\right] \frac{B^{2}}{\left(E_{1}+m_{1}\right)\left(E_{2}+m_{2}\right)} \\
\left.+\frac{\ln ^{\prime} \chi_{1} \chi_{2}}{r}\right\} u_{j 1 j}-\sqrt{(j(j+1)} \frac{\ln ^{\prime}\left(\chi_{1} / \chi_{2}\right)}{r} u_{j 0 j}=b^{2}(w) u_{j 1 j} .
\end{gathered}
$$

Note that for equal masses after extensive cancellations Eq.(D.56) simplifies to

$$
\left\{-\frac{d^{2}}{d r^{2}}+\frac{j(j+1)}{r^{2}}+2 \epsilon_{w} \mathcal{A}-\mathcal{A}^{2}\right\} u_{j 0 j}=b^{2}(w) u_{j 0 j}
$$


which can be solved exactly ${ }^{2}$ for $\mathcal{A}=-\alpha / \gamma$ in agreement with the chiral version presented in Ref.(16). We will not use the four coupled $l=j \pm 1$ equations for $j>0$. For the ${ }^{3} P_{0}$ state for QED Eq.(D.55) simplifies to

$$
\begin{gathered}
\left\{-\frac{d^{2}}{d r^{2}}+\frac{2}{r^{2}}+2 \epsilon_{w} \mathcal{A}-\mathcal{A}^{2}\right. \\
-\frac{1}{2} \partial^{2} \ln \left(\chi_{1} \chi_{2} G^{-1}\right)+\frac{1}{2}\left(\mathcal{G}^{\prime}\right)^{2}-\frac{1}{2} \ln ^{\prime} \chi_{1} \chi_{2} \mathcal{G}^{\prime}+\frac{1}{4}\left(\ln \chi_{1} \chi_{2}\right)^{2}-2 \frac{\mathcal{G}^{\prime}}{r} \\
+\left[\frac{1}{2} \ln ^{\prime} \chi_{1} \chi_{2} \mathcal{G}^{\prime}-\frac{1}{2}\left(\mathcal{G}^{\prime}\right)^{2}-\frac{1}{2} \partial^{2} \mathcal{G}+\frac{2 \mathcal{G}^{\prime}}{r}\right] \frac{B^{2}}{\left(E_{1}+m_{1}\right)\left(E_{2}+m_{2}\right)} \\
\left.+2 \frac{\left.\ln ^{\prime} \chi_{1}\right)(2}{r}\right\} u_{110}=b^{2}(w) u_{110} .
\end{gathered}
$$

For equal masses this becomes

$$
\begin{gathered}
\left\{-\frac{d^{2}}{d r^{2}}+\frac{2}{r^{2}}+2 \epsilon_{w} \mathcal{A}-\mathcal{A}^{2}\right. \\
-8 \frac{\mathcal{A}^{\prime}}{r w} \frac{1}{1-2 \mathcal{A} / w}+8\left(\frac{\mathcal{A}^{\prime}}{\mathfrak{w}^{\prime}}\right)^{2} \frac{1}{(1-2 \mathcal{A} / w)^{2}} \\
\left.+4 \frac{\partial^{2} \mathcal{A}}{w} \frac{1}{1-2 \mathcal{A} / w}\right\} u_{110}=b^{2}(w) u_{110}
\end{gathered}
$$

When $\mathcal{A}=-\alpha / r$ this becomes Eq.(7.1) in the text. Eq.(7.2) for the $j=l, s=0$ states for equal masses follows from our Eq.(D.56). 


\section{REFERENCES}

1. H. W Crater and P. Van Alstine, Phys. Rev. Lett. $\underline{53}, 1577$ (1984),

2. H. W. Crater and P. Van Alstine, Phys. Rev. D1 $\underline{37}, 1982$ (1988)

3. P. Van Alstine and H.W.Crater, J. Math. Phys. $\underline{23}, 1697$ (1982)

4. H. W. Crater and P. Van Alstine, Ann. Phys. (N.Y.) 148, 57 (1983).

5. H.W. Crater and P. Van Alstine, Phys. Rev. D15 $\underline{36}, 3007$ (1987)

6. P.A.M. Dirac, Lectures on Quantum Mechanics (Yeshiva University, New York, 1961).

7. P. Van Alstine, "Relativistic Two Body Problem from Singular Actions", Ph.D. Dissertation Yale University, 1976; M.Kalb and P. Van Alstine, Yale Reports Nos. C00-3075-146, 1976 and C00-3075$156,1976$.

8. I. T. Todorov, "Dynamics of Relativistic Point Particles as a Problem with Constraints", Dubna Joint Institute for Nuclear Research No. E2-10175, 1976; Ann. Inst. H. Poincare' A묘,207 (1978)

9. A. Komar, Phys. Rev. D $18,1881,1887$ (1978).

10. L. I. Horwitz and F. Rohrlich, Phys. Rev. D $\underline{24} 1528$ (1981).

11. S. L. Adler, Nuc. Phys. B217, 318 (1983), S. L. Adler and T. Piran, Phys. Lett., 117B, 91 (1982).

12. J. Richardson, Phys. Lett. $\underline{82 \mathrm{~B}} 272$, (1979).

13. H. W. Crater and P. Van Alstine, Phys. Rev. D30, 2585 (1984).

14. I. T. Todorov, in "Properties of the Fundamental Interactions," ed. by A. Zichichi (Editrice Compositori, Bologna, 1973), vol. 9, part C, pp. 953-979; Phys. Rev. D3, 2351 (1971).

15. Both QED and QCD at short distance (large momentum) have dynamics that arises from field theoretic amplitudes of the form 
$\gamma_{1}^{\mu} \gamma_{2 \mu} f\left(q^{2}\right)$. However, the nonabelian character of QCD leads to an $f$ that differs from that of QED and includes color matrices.

16. Peter Van Alstine and H. W. Crater, Phys. Rev. D34, 1932 (1986).

17. E.E. Salpeter, Phys. Rev. 87 328,(1952). Both M. A. Stroscio, Phys. Rep. C $\underline{22}, 215(1975)$ anc" Itzykson and Zuber Quantum Field Theory (McGraw-Hill Inc., New York, 1980) review this equation. Note that, as has been pointed out by R.Yaes,Phys. Rev.D3,3086 (1971), and F. Gross, Phys. Rev. C 26 , 2203 (1982) there are an infinite number of three dimensional rearrangements of the Bethe-Salpeter equation.

18. C. Y. Wong and R. L. Becker, Phys. Lett. 182B, 251 (1986). In numerical calculations in the term $V_{3}$ of this reference: $[\mathrm{m} /(E+2 m-$ $V)]\left[\left(r_{e} / r^{3}\right)(1+\vec{s} \cdot \vec{l})-\left(r_{e}^{2} / 6 r^{4}\right)\left(S_{12}-2 \sigma_{1} \cdot \sigma_{2}\right)\right]$, the term $\vec{s} \cdot \vec{l}$ was inadvertently programmed as $r_{e} \vec{s} \cdot \vec{l}$ where $r_{e}$ is fermis. The result of this reference was obtained by using this potential. When the erroneous factor is removed, although the potential shape is similar, there are no resonance states.

19. J. Schweppe et al., Phys. Rev. Lett. $\underline{51}, 2261$ (1983).

20. M. Clement et al., Phys, Lett. 137B, 41 (1983).

21. T. Cowan et al., Phys. Rev. Lett. 54, 1761 (1985).

22. H. Bokemeyer et al., GSI Annual Report, GSI-85-1, March 1985, p. 177 (unpublished).

23. H. Tserrtos et al., Phys. Lett. 162B, 273 (1985).

24. T. Cowan et al., Phys. Rev. Lett. $\underline{56}, 444$ (1986).

25. K. Maier et al, Z. Phys. A $\underline{326}, 527$ (1987); U. von Wimmersperg et al, Phys. Rev. Lett., 9ㅗ, 266 (1987); A. P. Mills Jr. and J. Levy, 
Phys. Rev. D, $\underline{36}, 707$ (1987). These three papers report direct searches for resonance in $e^{+} e^{-}$scattering.

26. "A Search for Low-Mass Short-Lived States Coupling to $e^{+} e^{-}$", $\mathrm{X}$. Wu et al, Bulletin of the American Physical Society $\underline{6}$, p. 1392 April, 1991, "A Search for Low-Mass Long-Lived States Coupling to $e^{+} e^{-n}$, S.D. Henderson et al, Bulletin of the American Physical Society $\underline{36}$, p. 1392, April, 1991.

27. Thus this resonance would be a "nonperturbative effect" by which we mean an effect that cannot be derived from a finite perturbatiou series of Feynman diagrams alone. A good example of such an effect is the Balmer formula, which requires a nonperturbative quantum mechanical manipulation of a perturbative QED potential ( the single photon exchange potential), using the Schrödinger equation. In diagrammatic language this amounts to an infinite iteration of the Born term. However, since the binding energy is much smaller than $m c^{2}$, this "nonperturbative effect" is nonrelativistic. In contrast, if the hypothesized purely quantum electrodynamic $\left(e^{+} e^{-}\right)$resonance exists, its existence is not only a "nonperturbative effect", but also a highly relativistic one because the resonance energy is $\sim m c^{2}$.

28. J.R. Spence and J.P. Vary, Phys.Lett B254, 1, (1991).

29. The collective minimal Todorov form is in general $2 m_{w} S+S^{2}+$ $2 \epsilon_{w} \mathcal{A}-\mathcal{A}^{2}+2 \epsilon_{w} \mathcal{V}-\mathcal{V}^{2}$ when we include a scalar and an additional time-like vector interactions. See the first part of Eqs.(4.21a-b).

30. B.L. Aneva, J.I. Krapchev, and V.A. Rizov, Bulg. J. Phys 2 409, (1975). V.A. Rizov and I.T. Todorov, Fiz. Elem. Chastits At. Yadre $\underline{6}, 669$ (1075) [translated in Sov. Jl. Part. Nucl. 6. 269 
(1975)].

31. H.A. Bethe and E. E. Salpeter, Quantum Mechanics of One and

Two Electron Atoms (Springer, Berlin, 1957).

32. W. Krolikowski, Acta Physica Polonica, B12, 891 (1981). Nonperturbative treatments of truncated version of the Breit equation have yielded the same results as a perturbative treatment of the same truncations but none of these have included the troublesome transverse photon parts. See J. Malenfant, Phys. Rev. A $\underline{43}, 1233$ (1991), T.C. Scott and J. Shertzer, "Accurate Finite Element Solutions to the Two-Body Dirac Equations" 4/91.

33. Recently, Wallace and Thayyullathil have numerically solved the Wallace-Mandelzweig equation but have been able to obtain the correct hyperfine splitting in positronium and muonium only if the coupling to the lower-lower portions of the wave function was eliminated. S.J. Wallace, "Two-Body Dirac Equations and Applications to Atomic Hyperfine Splittings" Lampf Workshop Proc. Nuclear and Particle Physics on the Light Cone Los Alamos, New Mexico (7/88) World Scientific, Singapore (1989).

34. H. Crater and D. Yang, J. Math. Phys. 32, 2374 (1991), , H. W. Crater and P. Van Alstine, "Restrictions imposed on relativistic twobody interactions by classical relativistic field theory", to appear in Phys. Rev. D

35. We point out that in most other covariant three dimensional approaches to the two-body problem in QED (see for example that of W. E. Caswell and G. P. Lepage, Phys. Rev. A18, 863,(1977) A18,810 (1978)) the Coulomb gauge is needed in order to avoid sum- 
ming an infinite number of diagrams. In the constraint equations, we are able to use the Feynman gauge to obtain the standard spectral results of atomic physics using the first Born amplitude alone.

36. H.W. Crater and P. Van Alstine, J. Math. Phys. 31, 1998 (1990)

37. The algebraic significance of the theta matrices in the dynamical description provided by Eqs.(2.18a-b) is that they ensure that the Dirac operators $\mathcal{S}_{10}$ and $\mathcal{S}_{20}$ are the operator square roots of the corresponding mass-shell operators $-\frac{1}{2}\left(p_{1}^{2}+m_{1}^{2}\right)$ and $-\frac{1}{2}\left(p_{2}^{2}+m_{2}^{2}\right)$.

38. H. Sazdjian, Phys. Rev. D1 $\underline{33}, 3401$ (1986), derives compatible twobody Dirac equations but from a different starting point without the use of supersymmetry.

39. Alternatively, the Sazdjian forms are equivalent to those of (2.25a-b) if in (2.27a-b) $\Delta$ is replaced by th $\Delta$ and $\psi \rightarrow \operatorname{ch} \Delta \psi$. Without this replacement, the Schrödinger-like equation that result from Sazdjian's $(2.27 \mathrm{a}-\mathrm{b})$ are much more complex than those that come from our $(2.25 \mathrm{a}-\mathrm{b})$.

40. H. Sazdjian, Phys. Lett. 156B, 381 (1985); Also F. Coester (private communication).

41. The Lippmann-Schwinger equation $T+V+V G T=0$ can also be written as $T+V+T G V=0$. From these two equations one obtains

$$
T-T^{\dagger}=-V G T+T^{\dagger} G^{\dagger} V=\left(T^{\dagger}+T^{\dagger} G^{\dagger} V\right) G T-T^{\dagger} G^{\dagger}(T+V G T)=T^{\dagger}\left(G-G^{\dagger}\right) T
$$

42. A.A. Logunov and A.N. Tavkhelidze, Nuovo Cimento $\underline{29}, 380$ (1963);

$$
\text { A.A. Logunov et al., ibid 30,134 (1963) }
$$

43. R. Blankenbecler and R. Sugar, Phys. Rev. 142,1051 (1966).

44. Notice that the space part of this vector potential is not Todorov's pseudolocal form ${ }^{14} \sim \frac{i x_{\perp}^{\mu} \alpha}{2 w r^{3}} I_{s}$ where $I_{s} x_{\perp}^{\mu}=-x_{\perp}^{\mu} I_{s}$. Note further 
that a scale change (as opposed to a phase transformation) can eliminate $\mathcal{V}_{\mu}$.

45. J. Schwinger, Particles, Sources, and Fields (Addison-Wesley, Reading, 1973), Vol. 2, pp.348-349.

46. H. Sazdjian, private communication.

47. In Ref.(16), two of us used a third method for finding solutions of the Dirac equations from solutions of the squared equations for the case of electrodynamics. This method, which uses the compatibility of the two constraints $\mathcal{S}_{1}$ and $\mathcal{S}_{2}$ written in the chiral gamma matrix representation, is not practical for general interactions for which the chiral representation does not lead to simplifications.

48. The doubly odd part of the quasipotential is distinctly different from the quasipotential in Refs. 4-5 (valid only for $j=l$ ) which involved no coupling. Eq.(4.22) gives one possible correction to Eq.(172) in Ref.5. Other possibilities are presented in Appendix D.

49. This scaling, and the ones below for scalar and time-like vector interaction, is a relativistic version of that occuring for the nonrelativistic Schödinger equation which allows the decoupling of effects of scale $m$ from those at the scale $\alpha m$.

50. For positronium our results agree with the standard results of Ref.(17.) without the annihilation diagram. Ref.(17) also gives the spectrum for the general hydrogenic (unequal mass) case but in the $j-j$ coupling scheme, rather than the $L S$ coupling scheme that we present here. This scheme is given in Ref.(30) but with a spectrum that does not have the correct $j=l$ spin mixing fine-structure even though the weak potential bound state equations given there are correct. The 
corrected spectrum appears in J. Connell, Phys. Rev. D43, 1393, (1991). Note that the equations Connell uses are similar to the Sazdjian equations in the Coulomb gauge. Our equation (Eq.(5.7)) uses the Feynman gauge.

51. P. Van Alstine and H. W. Crater, Phys. Rev. D $\underline{33}, 1037$ (1986).

52. H. W. Crater, J. Comp. Phys., 19,236 (1975)

53. H. W. Crater, Phys. Rev. D 12,1804 (1975)

54. Ref.(30) presents a perturbative treatment of this based on the Todorov equation. The constraint equation for this will be derived in a future publication which will include a nonperturbative as well as a perturbative treatment.

55. Note that this table and the one for muonium includes many more states than are actually observed. The reason that we include so many states is that we wish to test the validity of the strong potential form of the equations for the rich variety of quantum numbers observed for quarkonia. The actual experimental spectra for positronium and muonium are, unfortunately not as rich as those presented above. Of course for the ${ }^{3} S_{1}$ positronium states the experimental spectrum would include the effect of the annihilation diagram (which would avoid the artificial degeneracy in the table between the ${ }^{3} S_{1}$ and ${ }^{1} P_{1}$ states with the same $\left.n\right)$.

56. Alternatively, the fact that perturbative results do not require any coupling to the lower-lower components may seem surprising.

57. In this paper we do not addressing the question of the short distance behavior of the invariant $\mathcal{A}(r)$ which to lowest order is simply $-\alpha / r$. The additional corrections are dictated by higher order QED. Given 
$\mathcal{A}$, the way in which that $\mathcal{A}$ affects the strong-potential structures of the quasipotential in the wave equations $(4.21 \mathrm{a}-\mathrm{b})$ is fixed by the or ininal form (2.1a-b) of the wave equation through its dependence on $G(\mathcal{A})$.

58. This phenomenon can be made explicit by comparing the expectation value of the original $i \hat{r} \cdot p$ term, computed with and without the exact wave function, by integration by parts after an appropriate scale change.

59. D. G. Caldi and A. Chodos, Phys. Rev. D 362876 (1987), L.S. Celenza, V.K. Mishra, C.M. Shakin, and K.F. Liu, Phys. Rev. Lett. $\underline{57}, 55$ (1986);

60. J. B. Kogut, E. Dagotto and A. Kovic, Phys. Rev. Lett. 60772 (1988) ]

61. A. B. Balantekin, C. Bottcher, M. R. Strayer, and S. J. Lee, Phys. Rev. Lett. $\underline{55} 461$ (1985).

62. A. Schafer, J. Reinhard, B. Müller, and W. Greiner, J. Phys. G11, L69 (1985).

63. A. Chodos and L.C.R. Wijewardhana, Phys. Rev. Lett. $\underline{56}, 302$ (1986).

64. Note that the technique used in Appendix D, based on ideas presented in private communications with $\mathrm{H}$. Sazdjian, is different from that used in earlier ${ }^{4-5}$ work. This earlier work used a decoupling of the upper-upper component from the lower-lower component that was valid only for the $j=l$ states. The results in Appendix D reproduce the same results for the $j=l$ states and go on to give valid equations for the $j=l \pm 1$ states, leading to an uncoupled equa- 
tion for the ${ }^{3} P_{0}$ system distinctly different from that which would be obtained from the decoupled equations in the earlier work. The new $j=l \pm 1$ equations contain effective potentials (including the angular momentum barrier) for QED that are well defined for all values of $\alpha$ of interest in sharp contrast to those which would occur if the equations of Ref.(4-5) were applied to the the $j=l \pm 1$ states (for the ${ }^{3} P_{0}$ state the effective potential was singular for all values of $\alpha$ (unpublished results)).

65. F. Calogero, Variable Phase Approach to Potential Scattering, Academic Press, New York and London, (1967).

66. Thus our minimal interaction constraint equations show explicitly that there are circumstances where magnetic dipole forces (arising from the spin-orbit interactions) are more important than Coulomb forces and details how this interplay between Coulomb and magnetic dipole forces actually takes place in the quasipotential of (7.1) at distances on the order of $\alpha / m$. Note that there is a naive argument that shows how such short distance scales arise when magnetic dipole forces dominate. For circular motion with the magnetic moment perpendicular to the plane of the orbit, the force equation reduces to $m \gamma v^{2} / r=e \mu v / r^{3}$. Simple Bohr quantization leads to $L=e \mu / r=n \hbar$. Using the Bohr magneton yields $r=r_{e} / 2 n\left(r_{e}\right.$ is the classical electron radius) and $\left(\gamma v^{2}\right)=2 n^{2} / \alpha$. This is a highly relativistic motion with orbits not on the order of atomic dimensions as in the case when the Coulomb potential dominates, but on the order of nuclear dimensions.

67. Note that this short distance strong-potential behavior is distinctly 
different from the short distance behavior produced by the effective potential used by two of us in Ref.18. In that reference it was hypothesized that a pocket in an effective potential well may exist in a Schrödinger-like equation at about 1-2 fermis for the $\left(e^{+} e^{-}\right)$system in a ${ }^{3} P_{0}$ state. Using crude argv ments one can show how this pocket might come about for this state. First one has the ordinary ${ }^{3} P_{0}$ positronium well at about an Angstrom. Then at shorter distances the angular momentum barrier begins to rise. At much shorter distances ( a few fermis) this repulsive barrier may be overcome by the highly attractive $\frac{1}{r^{3}}$ spin-orbit potential in this state. The inner pocket is produced by a still shorter range repulsive $\frac{1}{r^{4}}$ potential produced by the square of the vector potential used in Ref.(18), $\vec{A}_{i}=\vec{\mu}_{j} \times\left(\vec{r}_{i}-\vec{r}_{j}\right) /\left|\vec{r}_{i}-\vec{r}_{j}\right|^{3}$, with $\vec{\mu}_{j}$ the magnetic moment of the $j t h$ particle. The effective interaction in the Schrödinger-like equation then contained an attractive $1 / r^{3}$ part and a repulsive $1 / r^{4}$ even for very small distances. But can we really expect such singular potentials to be valid at such short distances? It is one thing to use them as perturbations, but quite another to regard them seriously as effective potentials with substantial influences on the wave function. Our covariant constraint equations force those effective potentials to be moderated by relativistic effects at $r \sim \alpha / m$ and have substantial influence on the wave function. Without that influence the effect of the lower-lower component would not have produced the correct hyperfine splitting. The quasipotential in our constraint equations does have an attractive $1 / r^{3}$ part and a repulsive $1 / r^{4}$ part but at distances intermediate between a Fermi and an Angstrom, not at 
still shorter distances. Thus, our effective potential (including the angular momentum barrier) in the Schrödinger-like form Eqs.(7.1) of the two-body Dirac equations for QED, although attractive at short distance, is not deep or wide enough to support a resonance.

68. Without this elimination, the Bethe-Salpeter equation gives erroneous $O(1 / c)$ (as opposed to $O\left(1 / c^{2}\right)$ ) corrections to the non-relativistic results. It also has extra solutions (with no non-relativistic limit). In addition, without a truncation to a one time equation, the BetheSalpeter equation does not satisfy the requirement that it approach a one-body equation when one of the masses becomes very large, for kernels of finite order. G. C. Wick, Phys. Rev. 96,1124 (1954), R. E. Cutkosky, Phys. Rev. $\underline{96}, 1135$ (1954). I. T. Todorov, Lectures in Theoretical Physics XII, University of Colorado, 1969, W. Brittin and K. Mahanthappa, editors (Interscience, New York,1971). This combination of unfortunate circumstances for the four dimersional Bethe-Salpeter equation has recently been discussed by F. Gross in Ref.(17). He points out the paradox that the extra work that must be done to keep the relative time (or relative energy) as a variable seems to reward one with poorer answers.

69. V.B. Mandelzweig and S.J. Wallace, Phys. Lett.B197,469 (1988).

70. Moreover, Cooper and Jennings have recently rederived the twobody constraint equation (2.1a-b) by an Eikonal argument. E.D. Cooper and B.K. Jennings, Nucl. Phys. A483, 601 (1988).

71. By Fermi-Breit expansion we mean, for example, that obtained by applying the Chraplyvy transformation (a two-body Foldy-Wouthuysen transformation) to the three-dimensional Bethe-Salpeter equation. 
Stroscio in Ref.(17)

72. The Schrödinger-like form of the covariant constraint equations (4.21ab) for the fermion-antifermion system has a weak-potential approximation given in Eq.(5.9). A canonical transformation of the $O\left(1 / c^{2}\right)$ expansion of Eq.(5.9) (see Refs.13 and 34) then reproduces the Fermi-Breit equation, Eq.(9.5a-b).

73. This has been done for two spinless particles in Ref.13.

74. In Ref.(16), two of us found that in the case of singlet positronium solution of our full coupled sixteen component two-body Dirac equations (Eqs.(2.1a-b)) reduces to the solution of the minimal Todorov equation Eq.(7.2). Independently, Bohnert et al. (see G. Bohnert, R. Decker, A. Hornberg, H. Pilkuhn, H.G. Schlaile, Z. Phys. $\underline{2}$, 23 (1986)) found that their method leads to the minimal Todorov equation as a second order wave equation for singlet positronium.

75. R. Barbieri and E. Remiddi, Nucl. Phys. B141, 413 (1978).

76. The Schrödinger rearrangement (Eqs.(4.21a-b)) of our minimal interaction constraint equation Eqs.(2.1a-b) differs from the minimal Todorov equation by the appearance of strong potential terms. As shown in appendix D, those terms "miraculously" cancel for singlet positronium.

77. R. W. Childers, Phys. Rev. D杰,2902 (1982).

78. S. Godfrey and N. Isgur, Phys. Rev. D32, 189 (1985).

79. The authors thank H. Sazdjian for suggesting this useful change. 
TABLE I. Comparison between perturbatively and numerically calculated energy levels for positronium.

The first four columns list the quantum numbers $l, s, j, n$. In the fifth column is the number of coupled equations $N_{c}$ that were used to perform the numerical test. In the next column are the energy levels obtained from the perturbative expansions given in section V. The last column gives the difference between the perturbative and numerical calculations divided by $\mu \alpha^{4} / n^{3}$.

\begin{tabular}{|c|c|c|c|c|c|c|c|c|}
\hline$l$ & $s$ & $j$ & $n$ & $N_{c}$ & & perturbative & numerical & $\operatorname{diff} / \frac{\mu \alpha^{4}}{n^{3}}$ \\
\hline 0 & 0 & 0 & 1 & 1 & & -6.8033256279 & -6.8032861579 & $5.45 \mathrm{E}-02$ \\
\hline 0 & 0 & 0 & 1 & 2 & & -6.8033256279 & -6.8033256719 & $-6.08 \mathrm{E}-05$ \\
\hline J & 1 & 1 & 1 & 1 & & -6.8028426132 & -6.8028074990 & $4.84 \mathrm{E}-02$ \\
\hline 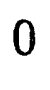 & 1 & 1 & 1 & 2 & $\mathrm{M}$ & -6.8028426132 & -6.8028082195 & 4.75E-02 \\
\hline 0 & 1 & 1 & 1 & 2 & $\mathrm{C}$ & -6.8028426132 & -6.8028239499 & $2.58 \mathrm{E}-02$ \\
\hline 0 & 1 & 1 & 1 & 4 & & -6.8028426132 & -6.8028426636 & $-6.97 \mathrm{E}-05$ \\
\hline U & 0 & 0 & 2 & 1 & & -1.7007875394 & -1.7007826068 & 5.45E-02 \\
\hline & 0 & 0 & 2 & 2 & & -1.7007875394 & -1.7007875467 & $-8.04 \mathrm{E}-05$ \\
\hline & 1 & 1 & 2 & 1 & & -1.7007271626 & -1.7007227741 & $4.85 \mathrm{E}-02$ \\
\hline & 1 & 1 & 2 & 2 & $M$ & -1.7007271626 & -1.7007228642 & 4.75E-02 \\
\hline 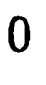 & 1 & 1 & 2 & 2 & $\mathrm{C}$ & -1.7007271626 & -1.7007248306 & $2.57 \mathrm{E}-02$ \\
\hline & 1 & 1 & 2 & 4 & & -1.7007271626 & -1.7007271700 & $-8.09 \mathrm{E}-05$ \\
\hline & 0 & 1 & 2 & 1 & & -1.7007271626 & -1.7007271630 & $-5.02 \mathrm{E}-06$ \\
\hline & 0 & 1 & 2 & 2 & & -1.7007271626 & -1.7007271630 & $-5.02 \mathrm{E}-06$ \\
\hline & 1 & 0 & 2 & 1 & & -1.7007573510 & -1.7007573538 & $-3.08 \mathrm{E}-05$ \\
\hline & 1 & 0 & 2 & 2 & & -1.7007573510 & -1.7007573563 & $-5.82 \mathrm{E}-05$ \\
\hline
\end{tabular}




$\begin{array}{lllllllll}1 & 1 & 1 & 2 & 1 & & -1.7007347097 & -1.7007347102 & -5.26 \mathrm{E}-06 \\ 1 & 1 & 1 & 2 & 2 & & -1.7007347097 & -1.7007347103 & -6.83 \mathrm{E}-06 \\ 1 & 1 & 2 & 2 & 1 & & -1.7007165966 & -1.7007165969 & -3.00 \mathrm{E}-06 \\ 1 & 1 & 2 & 2 & 2 & \mathrm{M} & -1.7007165966 & -1.7007165969 & -3.01 \mathrm{E}-06 \\ 1 & 1 & 2 & 2 & 2 & \mathrm{C} & -1.7007165966 & -1.7007165969 & -3.11 \mathrm{E}-06 \\ 1 & 1 & 2 & 2 & 4 & & -1.7007165966 & -1.7007165969 & -3.27 \mathrm{E}-06 \\ 0 & 0 & 0 & 3 & 1 & & -0.7558959994 & -0.7558945397 & 5.44 \mathrm{E}-02 \\ 0 & 0 & 0 & 3 & 2 & & -0.7558959994 & -0.7558960034 & -1.47 \mathrm{E}-04 \\ 0 & 1 & 1 & 3 & 1 & & -0.7558781100 & -0.7558768116 & 4.84 \mathrm{E}-02 \\ 0 & 1 & 1 & 3 & 2 & \mathrm{M} & -0.7558781100 & -0.7558768383 & 4.74 \mathrm{E}-02 \\ 0 & 1 & 1 & 3 & 2 & \mathrm{C} & -0.7558781100 & -0.7558774209 & 2.57 \mathrm{E}-02 \\ 0 & 1 & 1 & 3 & 4 & & -0.7553781100 & -0.7558781140 & -1.51 \mathrm{E}-04 \\ 1 & 0 & 1 & 3 & 1 & & -0.7558781100 & -0.7558781115 & -5.71 \mathrm{E}-05 \\ 1 & 0 & 1 & 3 & 2 & & -0.7558781100 & -0.7558781115 & -5.71 \mathrm{E}-05 \\ 1 & 1 & 0 & 3 & 1 & & -0.7558870547 & -0.7558870569 & -8.26 \mathrm{E}-05 \\ 1 & 1 & 0 & 3 & 2 & & -0.7558870547 & -0.7558870577 & -1.12 \mathrm{E}-04 \\ 1 & 1 & 1 & 3 & 1 & & -0.7558803462 & -0.7558803477 & -5.71 \mathrm{E}-05 \\ 1 & 1 & 1 & 3 & 2 & & -0.7558803462 & -0.7558803478 & -6.02 \mathrm{E}-05 \\ 1 & 1 & 2 & 3 & 1 & & -0.7558749793 & -0.7558749808 & -5.47 \mathrm{E}-05 \\ 1 & 1 & 2 & 3 & 2 & \mathrm{M} & -0.7558749793 & -0.7558749808 & -5.47 \mathrm{E}-05 \\ 1 & 1 & 2 & 3 & 2 & \mathrm{C} & -0.7558749793 & -0.7558749808 & -5.48 \mathrm{E}-05 \\ 1 & 1 & 2 & 3 & 4 & & -0.7558749793 & -0.7558749808 & -5.50 \mathrm{E}-05 \\ 2 & 0 & 2 & 3 & 1 & & -0.7558745321 & -0.7558745324 & -1.16 \mathrm{E}-05 \\ 2 & 0 & 2 & 3 & 2 & & -0.7558745321 & -0.7558745324 & -1.16 \mathrm{E}-05 \\ 2 & 1 & 1 & 3 & 1 & & -0.7558767683 & -0.7558767686 & -1.29 \mathrm{E}-05 \\ 2 & 1 & 1 & 3 & 2 & \mathrm{M} & -0.7558767683 & -0.7558767686 & -1.29 \mathrm{E}-05\end{array}$




$\begin{array}{lllllllll}2 & 1 & 1 & 3 & 2 & \mathrm{C} & -0.7558767683 & -0.7558767686 & -1.29 \mathrm{E}-05 \\ 2 & 1 & 1 & 3 & 4 & & -0.7558767683 & -0.7558767686 & -1.29 \mathrm{E}-05 \\ 2 & 1 & 2 & 3 & 1 & & -0.7558749793 & -0.7558749797 & -1.17 \mathrm{E}-05 \\ 2 & 1 & 2 & 3 & 2 & & -0.7558749793 & -0.7558749797 & -1.17 \mathrm{E}-05 \\ 2 & 1 & 3 & 3 & 1 & & -0.7558732543 & -0.7558732546 & -1.13 \mathrm{E}-05 \\ 2 & 1 & 3 & 3 & 2 & \mathrm{M} & -0.7558732543 & -0.7558732546 & -1.13 \mathrm{E}-05 \\ 2 & 1 & 3 & 3 & 2 & \mathrm{C} & -0.7558732543 & -0.7558732546 & -1.13 \mathrm{E}-05 \\ 2 & 1 & 3 & 3 & 4 & & -0.7558732543 & -0.7558732546 & -1.14 \mathrm{E}-05\end{array}$


TABLE II. Comparison between perturbatively and numerically calculated energy levels for muonium.

The first four columns list the quant'm numbers $l, s, j, n$. In the fifth column is the number of coupled equations $N_{c}$ that were used to perform the numerical test. In the next column are the energy levels obtained from the perturbative expansions given in section V. The last column gives the difference between the perturbative and numerical calculations divided by $\mu \alpha^{4} / n^{3}$.

$\begin{array}{llllllccc}l & s & j & n & N_{c} & & \text { perturbative } & \text { numerical } & \text { diff } / \frac{\mu \alpha^{4}}{n^{3}} \\ 0 & 0 & 0 & 1 & 1 & & -13.5404101578 & -13.540410298 & 8.87 \mathrm{E}-05 \\ 0 & 0 & 0 & 1 & 2 & & -13.5404101578 & -13.5404101581 & -2.44 \mathrm{E}-07 \\ 0 & 1 & 1 & 1 & 1 & & -13.5403917381 & -13.5403915568 & 1.26 \mathrm{E}-04 \\ 0 & 1 & 1 & 1 & 2 & \mathrm{M} & -13.5403917381 & -13.5403915646 & 1.20 \mathrm{E}-04 \\ 0 & 1 & 1 & 1 & 2 & \mathrm{C} & -13.5403917381 & -13.5403916417 & 6.69 \mathrm{E}-05 \\ 0 & 1 & 1 & 1 & 4 & & -13.5403917381 & -13.5403917373 & 5.52 \mathrm{E}-07 \\ 0 & 0 & 0 & 2 & 1 & & -3.3851119169 & -3.3851119037 & 7.32 \mathrm{E}-05 \\ 0 & 0 & 0 & 2 & 2 & & -3.3851119169 & -3.3851119197 & -1.59 \mathrm{E}-05 \\ 0 & 1 & 1 & 2 & 1 & & -3.3851096144 & -3.3851095945 & 1.11 \mathrm{E}-04 \\ 0 & 1 & 1 & 2 & 2 & \mathrm{M} & -3.3851096144 & -3.3851095954 & 1.05 \mathrm{E}-04 \\ 0 & 1 & 1 & 2 & 2 & \mathrm{C} & -3.3851096144 & -3.3851096051 & 5.18 \mathrm{E}-05 \\ 0 & 1 & 1 & 2 & 4 & & -3.3851096144 & -3.3851096170 & -1.45 \mathrm{E}-05 \\ 1 & 0 & 1 & 2 & 1 & & -3.3850653181 & -3.3850801475 & -8.23 \mathrm{E}-02 \\ 1 & 0 & 1 & 2 & 2 & \mathrm{M} & -3.3850653181 & -3.3850801475 & -8.23 \mathrm{E}-02 \\ 1 & 0 & 1 & 2 & 2 & \mathrm{C} & -3.3850653181 & -3.3850653187 & -3.35 \mathrm{E}-06 \\ 1 & 0 & 1 & 2 & 4 & & -3.3850653181 & -3.3850653187 & -3.35 \mathrm{E}-06\end{array}$




$\begin{array}{lllllllll}1 & 1 & 0 & 2 & 1 & & -3.3851107657 & -3.3851107678 & -1.20 \mathrm{E}-05 \\ 1 & 1 & 0 & 2 & 2 & & -3.3851107657 & -3.3851107678 & -1.22 \mathrm{E}-05 \\ 1 & 1 & 1 & 2 & 1 & & -3.3851099973 & -3.3850951694 & 8.23 \mathrm{E}-02 \\ 1 & 1 & 1 & 2 & 2 & \mathrm{M} & -3.3851099973 & -3.3850951694 & 8.23 \mathrm{E}-02 \\ 1 & 1 & 1 & 2 & 2 & \mathrm{C} & -3.3851099973 & -3.3851099994 & -1.15 \mathrm{E}-05 \\ 1 & 1 & 1 & 2 & 4 & & -3.3851099973 & -3.3851099994 & -1.16 \mathrm{E}-05 \\ 1 & 1 & 2 & 2 & 1 & & -3.3850650102 & -3.3850650108 & -3.32 \mathrm{E}-06 \\ 1 & 1 & 2 & 2 & 2 & \mathrm{M} & -3.3850650102 & -3.3850650108 & -3.32 \mathrm{E}-06 \\ 1 & 1 & 2 & 2 & 2 & \mathrm{C} & -3.3850650102 & -3.3850650108 & -3.32 \mathrm{E}-06 \\ 1 & 1 & 2 & 2 & 4 & & -3.3850650102 & -3.3850650108 & -3.32 \mathrm{E}-06 \\ 0 & 0 & 0 & 3 & 1 & & -1.5044889089 & -1.5044889088 & 3.43 \mathrm{E}-06 \\ 0 & 0 & 0 & 3 & 2 & & -1.5044889089 & -1.5044889135 & -8.57 \mathrm{E}-05 \\ 0 & 1 & 1 & 3 & 1 & & -1.5044882267 & -1.5044882246 & 4.08 \mathrm{E}-05 \\ 0 & 1 & 1 & 3 & 2 & \mathrm{M} & -1.5044882267 & -1.5044882248 & 3.54 \mathrm{E}-05 \\ 0 & 1 & 1 & 3 & 2 & \mathrm{C} & -1.5044882267 & -1.5044882277 & -1.80 \mathrm{E}-05 \\ 0 & 1 & 1 & 3 & 4 & & -1.5044882267 & -1.5044882312 & -8.43 \mathrm{E}-05 \\ 1 & 0 & 1 & 3 & 1 & & -1.5044751019 & -1.5044794986 & -8.23 \mathrm{E}-02 \\ 1 & 0 & 1 & 3 & 2 & \mathrm{M} & -1.5044751019 & -1.5044794986 & -8.23 \mathrm{E}-02 \\ 1 & 0 & 1 & 3 & 2 & \mathrm{C} & -1.5044751019 & -1.5044751048 & -5.51 \mathrm{E}-05 \\ 1 & 0 & 1 & 3 & 4 & & -1.5044751019 & -1.5044751048 & -5.47 \mathrm{E}-05 \\ 1 & 1 & 0 & 3 & 1 & & -1.5044885678 & -1.5044885713 & -6.42 \mathrm{E}-05 \\ 1 & 1 & 0 & 3 & 2 & & -1.5044885678 & -1.5044885713 & -6.44 \mathrm{E}-05 \\ 1 & 1 & 1 & 3 & 1 & & -1.5044883402 & -1.5044839495 & 8.22 \mathrm{E}-02 \\ 1 & 1 & 1 & 3 & 2 & \mathrm{M} & -1.5044883402 & -1.5044839495 & 8.22 \mathrm{E}-02 \\ 1 & 1 & 1 & 3 & 2 & \mathrm{C} & -1.5044883402 & -1.5044883436 & -6.37 \mathrm{E}-05 \\ 1 & 1 & 1 & 3 & 4 & & -1.5044883402 & -1.5044883436 & -6.37 \mathrm{E}-05\end{array}$




$\begin{array}{lllllllll}1 & 1 & 2 & 3 & 1 & & -1.5044750107 & -1.5044750136 & -5.52 \mathrm{E}-05 \\ 1 & 1 & 2 & 3 & 2 & \mathrm{M} & -1.5044750107 & -1.5044750136 & -5.52 \mathrm{E}-05 \\ 1 & 1 & 2 & 3 & 2 & \mathrm{C} & -1.5044750107 & -1.5044750136 & -5.52 \mathrm{E}-05 \\ 1 & 1 & 2 & 3 & 4 & & -1.5044750107 & -1.5044750136 & -5.52 \mathrm{E}-05 \\ 2 & 0 & 2 & 3 & 1 & & -1.5044706145 & -1.5044723749 & -3.30 \mathrm{E}-02 \\ 2 & 0 & 2 & 3 & 2 & \mathrm{M} & -1.5044706145 & -1.5044723749 & -3.30 \mathrm{E}-02 \\ 2 & 0 & 2 & 3 & 2 & \mathrm{C} & -1.5044706145 & -1.5044706151 & -1.13 \mathrm{E}-05 \\ 2 & 0 & 2 & 3 & 4 & & -1.5044706145 & -1.5044706151 & -1.13 \mathrm{E}-05 \\ 2 & 1 & 1 & 3 & 1 & & -1.5044750789 & -1.5044750795 & -1.22 \mathrm{E}-05 \\ 2 & 1 & 1 & 3 & 2 & \mathrm{M} & -1.5044750789 & -1.5044750795 & -1.22 \mathrm{E}-05 \\ 2 & 1 & 1 & 3 & 2 & \mathrm{C} & -1.5044750789 & -1.5044750795 & -1.22 \mathrm{E}-05 \\ 2 & 1 & 1 & 3 & 4 & & -1.5044750789 & -1.5044750795 & -1.22 \mathrm{E}-05 \\ 2 & 1 & 2 & 3 & 1 & & -1.5044750242 & -1.5044732651 & 3.29 \mathrm{E}-02 \\ 2 & 1 & 2 & 3 & 2 & \mathrm{M} & -1.5044750242 & -1.5044732651 & 3.29 \mathrm{E}-02 \\ 2 & 1 & 2 & 3 & 2 & \mathrm{C} & -1.5044750242 & -1.5044750249 & -1.21 \mathrm{E}-05 \\ 2 & 1 & 2 & 3 & 4 & & -1.5044750242 & -1.5044750249 & -1.21 \mathrm{E}-05 \\ 2 & 1 & 3 & 3 & 1 & & -1.5044705793 & -1.5044705799 & -1.14 \mathrm{E}-05 \\ 2 & 1 & 3 & 3 & 2 & \mathrm{M} & -1.5044705793 & -1.5044705799 & -1.14 \mathrm{E}-05 \\ 2 & 1 & 3 & 3 & 2 & \mathrm{C} & -1.5044705793 & -1.5044705799 & -1.14 \mathrm{E}-05 \\ 2 & 1 & 3 & 3 & 4 & & -1.5044705793 & -1.5044705799 & -1.14 \mathrm{E}-05\end{array}$


TABLE III. Comparison between perturbatively and numerically calculated energy levels for scalar interactions

The first four columns list the quantum numbers $l, s, j, n$. In the fifth column is the number of coupled equations $N_{c}$ that were used to perform the numerical test. In the next column are the energy levels obtained from the perturbative expansions given in section V. The last column gives the difference between the perturbative and numerical calculations divided by $\mu \alpha^{4} / n^{3}$.

\begin{tabular}{|c|c|c|c|c|c|c|c|c|}
\hline$l$ & $s$ & $j$ & $n$ & $N_{c}$ & & perturbative & numerical & $\operatorname{diff} / \frac{\mu \alpha^{4}}{n^{3}}$ \\
\hline 0 & 0 & 0 & 1 & 1 & & -6.8024199753 & -6.8023461124 & $0.10 \mathrm{E}-00$ \\
\hline 0 & 0 & 0 & 1 & 2 & & -6.8024199753 & -6.8024197886 & $2.58 \mathrm{E}-04$ \\
\hline 0 & 1 & 1 & 1 & 1 & & -6.8024199753 & -6.8023461124 & $0.11 \mathrm{E}-00$ \\
\hline 0 & 1 & 1 & 1 & 2 & M & -6.8024199753 & -6.8023530264 & $9.24 \mathrm{E}-02$ \\
\hline 0 & 1 & 1 & 1 & 2 & $\mathrm{C}$ & -6.8024199753 & -6.8023461124 & $0.11 \mathrm{E}-00$ \\
\hline 0 & 1 & 1 & 1 & 4 & & -6.8024199753 & -6.8024197446 & $3.18 \mathrm{E}-04$ \\
\hline
\end{tabular}


TABLE IV. Comparison between perturbatively and numerically calculated energy levels for time-like interactions

The first four columns list the quantum numbers $l, s, j, n$. In the fifth column is the number of coupled equations $N_{c}$ that were used to perform the numerical test. In the next column are the energy levels obtained from the perturbative expansions given in section V. The last column gives the difference between the perturbative and numerical calculations divided by $\mu \alpha^{4} / n^{3}$.

$\begin{array}{llllllccc}l & s & j & n & N_{c} & & \text { perturbative } & \text { numerical } & \operatorname{diff} / \frac{\mu \alpha^{4}}{n^{3}} \\ 0 & 0 & 0 & 1 & 1 & & -6.8031444973 & -6.8030849884 & 8.21 \mathrm{E}-02 \\ 0 & 0 & 0 & 1 & 2 & & -6.8031444973 & -6.8031443059 & 2.64 \mathrm{E}-04 \\ 0 & 1 & 1 & 1 & 1 & & -6.8031444973 & -6.8030849884 & 8.21 \mathrm{E}-02 \\ 0 & 1 & 1 & 1 & 2 & \mathrm{M} & -6.8031444973 & -6.8030907008 & 7.43 \mathrm{E}-02 \\ 0 & 1 & 1 & 1 & 2 & \mathrm{C} & -6.8031444973 & -6.8030849884 & 8.24 \mathrm{E}-02 \\ 0 & 1 & 1 & 1 & 4 & & -6.8031444973 & -6.8031442880 & 2.89 \mathrm{E}-04\end{array}$




\section{Figure Captions}

Fig. 1a The effective potentials $\Phi\left({ }^{1} P_{1}\right)+\frac{2}{r^{2}}$ (upper line) and $\Phi\left({ }^{3} P_{0}\right)+$ $\frac{2}{r^{2}}$ (lower line) in units of $\mathrm{MeV}^{2}$ as functions of $r$ in fermis.

Fig. 1b The effective potential $\Phi\left({ }^{3} P_{0}\right)+\frac{2}{r^{2}}$ in units of $M e V^{2}$ versus $r$ in fermis. 

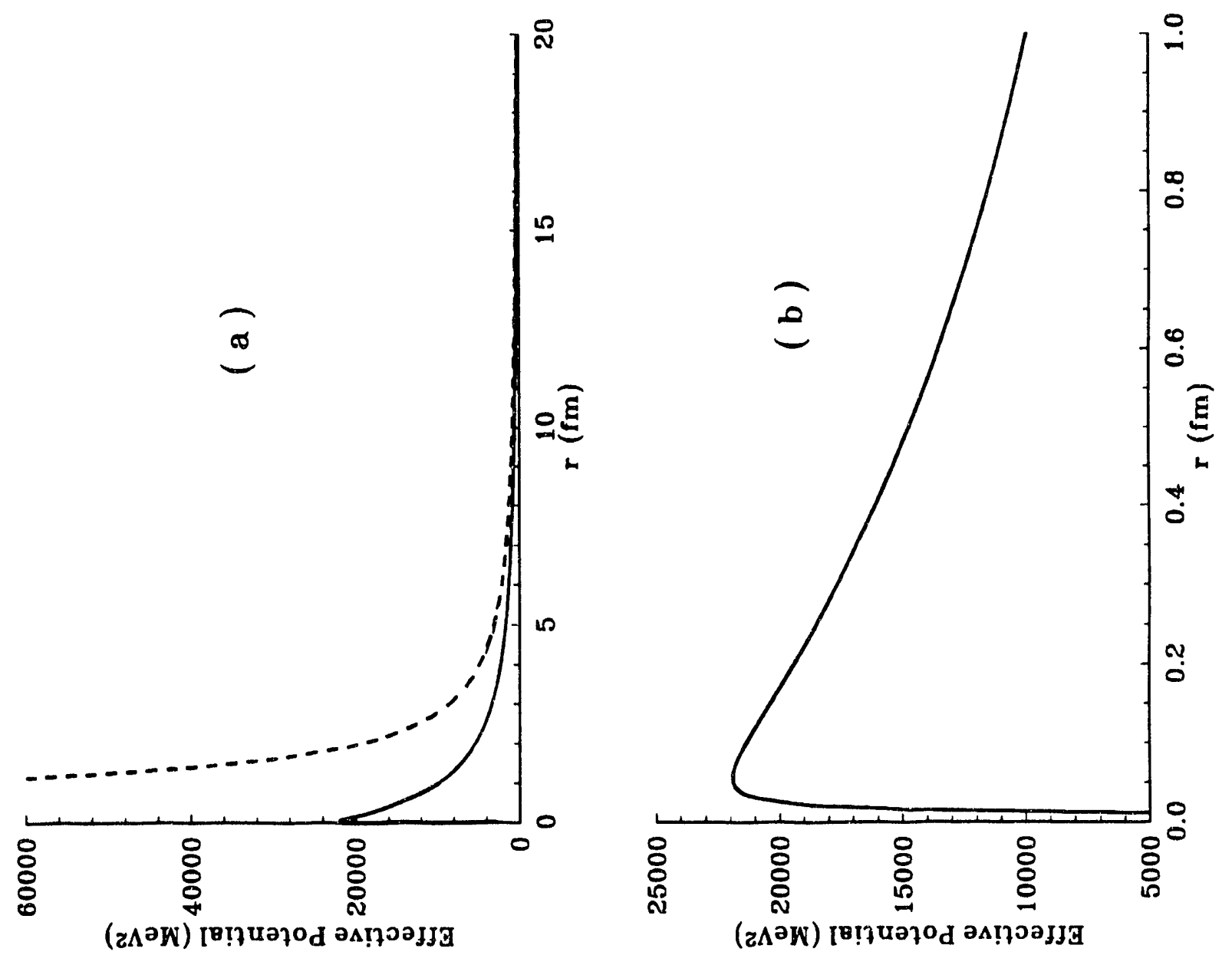
ORNL/TM-12122

\section{INTERNAL DISTRIBUTION}

1. B. R. Appleton

2. J. B. Ball

3. F. E. Barnes

4. J. R. Beene

5. F. E. Bertrand

6. C. Bottcher

7. K.T.R. Davies

8. J. D. Garrett

9. J. H. Macek

10. F. Plasil
11. G. R. Satchler

12. D. Shapira

13. M. R. Strayer

14-29. C. Y. Wong

30. Central Research Library

31. Document Reference Section

32-33. Laboratory Records Department

34. Laboratory Records Department - RC

35. ORNL Patent Office

\section{EXTERNAL DISTRIBUTION}

36. R. Barbieri, Dept. of Physics, Pisa University, Langarna Pacinoti 43, 56100 Pisa, Italy

37. A. O. Barut, Dept. of Physics, University of Colorado, Boulder, CO 80309

38. R. L. Becker, 2780 Oak Ridge Turnpike, Oak Ridge, TN 37830

39. J. Bijtebier, Theoretische Fysica, Vrije Universiteit Brussels, Fakulteit der Wetenschappen, Pleinlaan 2, B-1050 Brussels, Belgium

40. S. J. Brodsky, Linear Accelerator Center, P. O. Box 4349, Stanford, CA 94305

41. W. E. Caswell, 8138 Hartford Avenue, Silver Spring, MD 20910

42. H. W. Crater, University of Tennessee Space Institute, Tullahoma, TN 37388

43. G. W. Erickson, Dept. of Physics, University of California, Davis, CA 95616

44. G. Feinberg, Dept. of Physics, Box 60, Columbia University, New York, NY 10027

45. D. H. Feng, Dept. of Physics \& Atmospheric Science, Drexel University, Philadelphia, PA 19104

46. L. P. Fulcher, Jr., Dept. of Physics, Bowling Green State University, Bowling Green, OH 43403

47. J. S. Greenberg, Dept. of Physics, Yale University, P. O. Box 6666, New Haven, CT 06511

48. F. L. Gross, Dept. of Physics, College of William \& Mary, Williamsburg, VA 23185

49. H. Grotch, Dept. of Physics, Pennsylvania State University, 104 Davey Lab., University Park, PA 16802

50. S. N. Gupta, Dept. of Physics, Wayne State University, Detroit, MI 48202

51. N. Isgur, Theory Group, CEBAF, 12000 Jefferson Ave., Newport News, VA 23606

52. H. Ito, Dept. of Physics, Faculty of Science and Technology, Kinki University, Higashi-Osaka, 577, Japan

53. Rocio Jauregui, Instituto de Fisica, U.N.A.M., Apdo Postal 20-364, Mexico 01000, Mexico, D.F.

54. I. Y. Lee, Lawrence Berkeley Laboratory, Berkeley, CA 94720 
55. G. P. Lepage, Newman Laboratory of Nuclear Studies, Cornell University, Ithaca, NY 14853

56. L. Lusanna, Dipt. di Fisica, Sezione INFN di Firenze, Largo E. Fermi 2, Arcetri, 50125 Firenze, Italy

57. J. B. McGrory, Division of Nuclear Physics, Department of Energy, ER-23 G312/GTN, Washington, DC 20585

58. A. N. Mitra, Dept. of Physics, University of Delhi, Delhi, India

59. M. Moshinsky, Instituto de Fisica, Apartado Postal 20-364, Delegacion Alvargo Obregon, 01000 Mexico, D.F.

60. E. Remiddi, Dipt. di Fisica, Bologna University, via Zambeni 33, 40126 Bologna, Italy

61. H. Sazdjian, Division de Physique Théorique, Institut de Physique Nucléaire, Université Paris XI, 91406 Orsay Cedex, France

62. J. Shertzer, Dept. of Physics, College of the Holy Cross, Worcester, MA 01610

63. M. A. Stroscio, Electronics Division, Army Research Office, P. O. Box 12211, Research Triangle Park, NC 27709

64. J. Sucher, Dept. of Physics \& Astronomy, University of Maryland, College Park, MD 20742

65. I. Todorov, Physical Institute of Bulgarian Academy of Science, Sofia, Bulgaria

66. P. Van Alstine, 12474 Sunny Glenn Drive, Moorpark, CA 93021

67. J. P. Vary, Dept. of Physics, Iowa State University, Ames, IA 50011

68. S. J. Wallace, Dept. of Physics \& Astronomy, University of Maryland, College Park, MD 20742

69. D. R. Yennie, Newman Laboratory of Nuclear Studies, Cornell University, Ithaca, NY 14853

70. Office of Assistant Manager for Energy Research and Development, Department of Energy, Oak Ridge Operations Office, Oak Ridge, TN 37831

71-72. Office of Scientific and Technology Information, Department of Energy, Oak Ridge Operations Office, Oak Ridge, TN 37831 

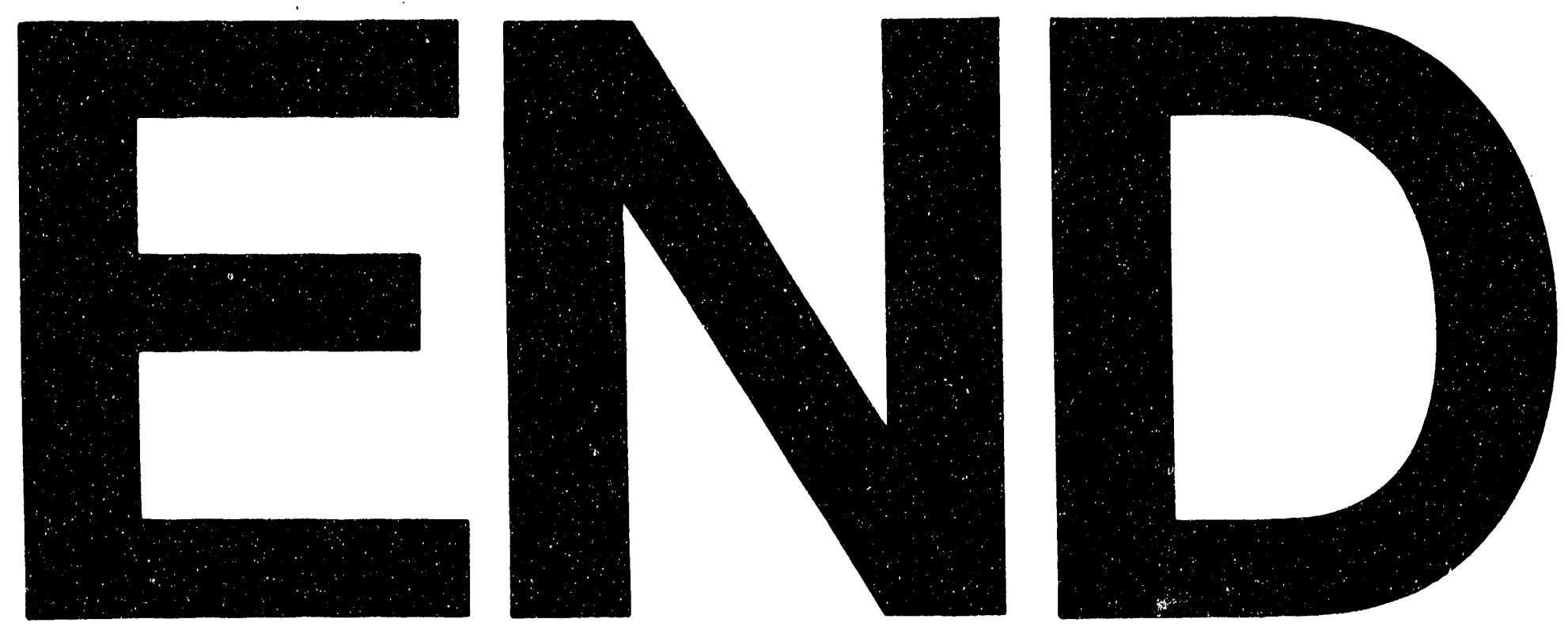

1
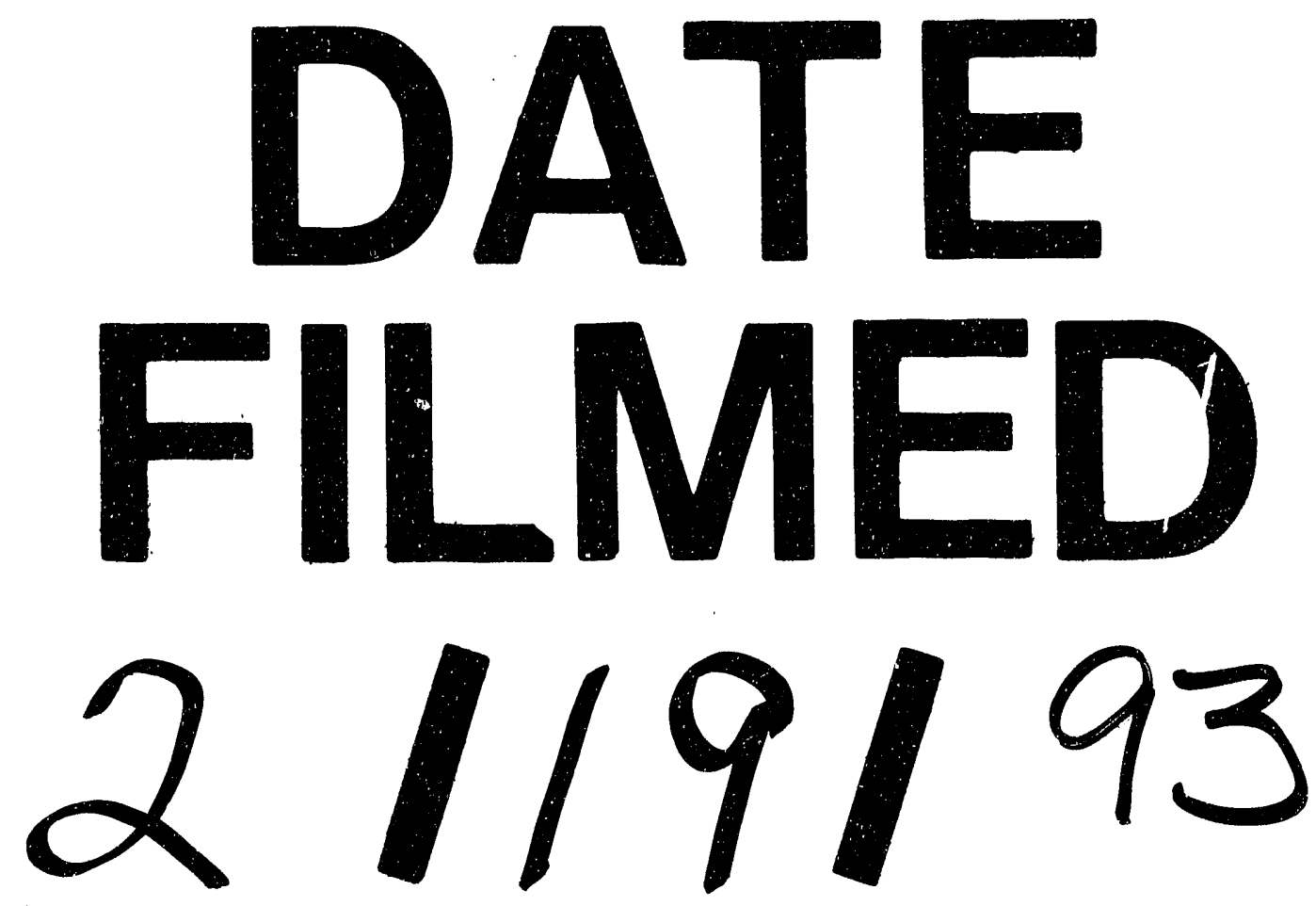
